This item was submitted to Loughborough's Research Repository by the author.

Items in Figshare are protected by copyright, with all rights reserved, unless otherwise indicated.

\title{
Hybrid energy harvesting technology: From materials, structural design, system integration to applications
}

\author{
PLEASE CITE THE PUBLISHED VERSION
}

https://doi.org/10.1016/j.rser.2020.110473

\section{PUBLISHER}

Elsevier BV

VERSION

AM (Accepted Manuscript)

\section{PUBLISHER STATEMENT}

This paper was accepted for publication in the journal Renewable and Sustainable Energy Reviews and the definitive published version is available at https://doi.org/10.1016/j.rser.2020.110473

\section{LICENCE}

CC BY-NC-ND 4.0

\section{REPOSITORY RECORD}

Liu, Huicong, Hailing Fu, Lining Sun, Chengkuo Lee, and Eric M Yeatman. 2020. "Hybrid Energy Harvesting Technology: From Materials, Structural Design, System Integration to Applications”. Loughborough University. https://hdl.handle.net/2134/13299683.v1. 


\section{Hybrid Energy Harvesting Technology: From Materials, Structural Design, System Integration to Applications}

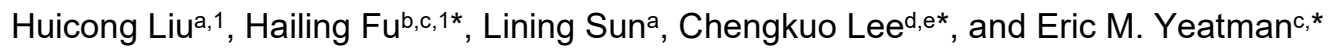

${ }^{a}$ School of Mechanical and Electric Engineering, Jiangsu Provincial Key Laboratory of Advanced Robotics, Soochow University, Suzhou 215123, China

${ }^{b}$ Wolfson School of Mechanical, Electrical and Manufacturing Engineering, Loughborough University, LE11 3TU, United Kingdom

${ }^{c}$ Department of Electrical and Electronic Engineering, Imperial College London, London, SW7 2AZ, United Kingdom

${ }^{d}$ Department of Electrical \& Computer Engineering, National University of Singapore, 4 Engineering Drive 3, Singapore, 117576, Singapore

${ }^{e}$ National University of Singapore Suzhou Research Institute (NUSRI), Suzhou Industrial Park, Suzhou, 215123, China

*Corresponding authors:

h.fu@,lboro.ac.uk (H. Fu); elelc@nus.edu.sg (C. Lee); e.yeatman@imperial.ac.uk (E. Yeatman)

${ }^{1}$ These authors contributed equally to this work. 


\begin{abstract}
:
The last decade has witnessed significant advances in energy harvesting technology for the realization of self-charging electronics and self-powered wireless sensor nodes (WSNs). To conquer the energy-insufficiency issue of a single energy harvester, hybrid energy harvesting systems have been proposed in recent years. Hybrid harvesting includes not only scavenging energy from multiple sources, but also converting energy into electricity by multiple types of transduction mechanisms. A reasonable hybridization of multiple energy conversion mechanisms not only improves the space utilization efficiency but can also boost the power output significantly. Given the continuously growing trend of hybrid energy harvesting technology, herein we present a comprehensive review of recent progress and representative works, especially focusing on vibrational and thermal energy harvesters which play the dominant role in hybrid energy harvesting. The working principles and typical configurations for piezoelectric, electromagnetic, triboelectric, thermoelectric and pyroelectric transduction effects are briefly introduced. On this basis, a variety of hybrid energy harvesting systems, including mechanisms, configurations, output performance and advantages, are elaborated. Comparisons and perspectives on the effectiveness of hybrid vibrational and thermal harvesters are provided. A variety of potential application prospects of the hybrid systems are discussed, including infrastructure health monitoring, industry condition monitoring, smart transportation, human healthcare monitoring, marine monitoring systems, and aerospace engineering, towards the future Internet-of-Things (IoT) era.
\end{abstract}

Key words: Hybrid energy harvesting, piezoelectric, electromagnetic, triboelectric, thermoelectric, pyroelectric

Word count: 11,567 


\section{Highlights}

- A systematic and comprehensive review of hybrid energy harvesting systems.

- Piezoelectric, electromagnetic, triboelectric, thermoelectric and pyroelectric effects and their use are reviewed in hybrid devices.

- Typical configurations, mechanisms and their performance are discussed and compared.

- Typical applications for hybrid energy harvesting mechanisms and systems are discussed and summarized.

- Future opportunities and directions are mapped following the discussion on existing challenges and applications. 


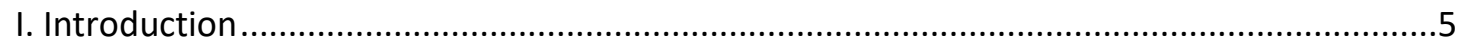

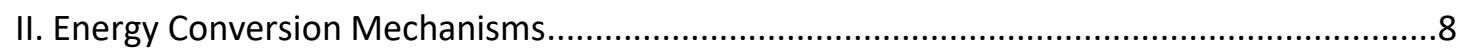

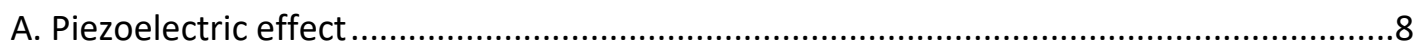

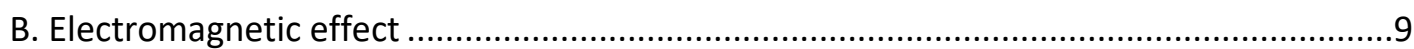

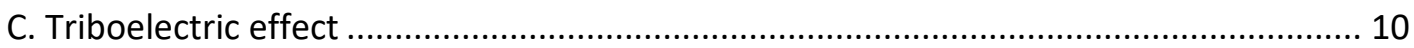

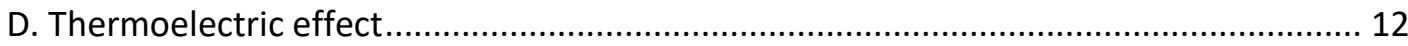

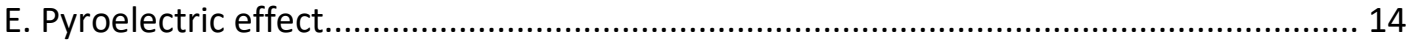

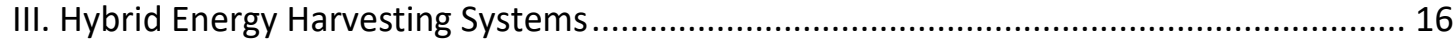

A. Piezoelectric-electromagnetic (PE-EM) hybrid systems.............................................. 16

B. Piezoelectric-triboelectric (PE-TE) hybrid systems ................................................... 19

C. Electromagnetic-triboelectric (EM-TE) hybrid systems............................................... 22

D. Piezoelectric-electromagnetic-triboelectric (PE-EM-TE) hybrid system .......................... 25

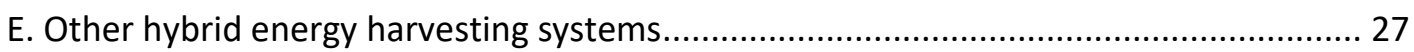

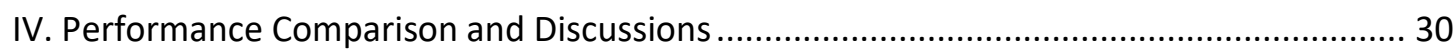

V. Application Prospects for Hybrid Energy Harvesting Systems ............................................ 34

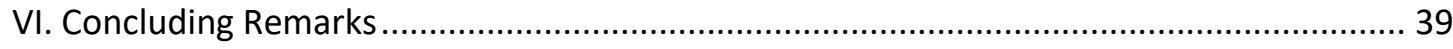

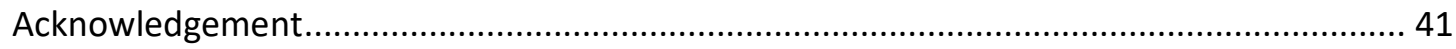

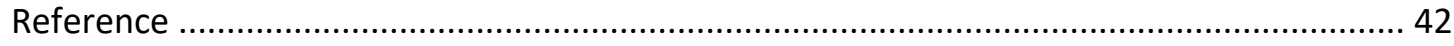

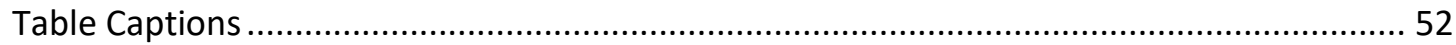

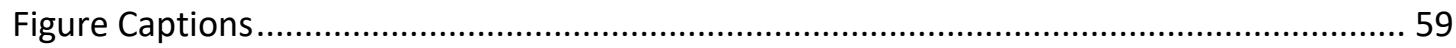




\section{Introduction}

With the rapid growth of the industrial economy and world population, conventional energy sources such as fossil fuels are no longer sufficient to meet energy demands. The utilization of renewable/sustainable energy sources, in the form of light, heat, wind, wave, vibration, rotation, etc., becomes an effective and promising solution to deal with the global energy crisis [1-5]. Over the past decade, a large number of researches and efforts have been involved to environmental energy harvesting techniques. Such energy sources are utilized to generate macro scale ( $>$ W level) electricity for industrial and household use, and hence alleviate the shortage of local power supply. In the meantime, energy harvesting research focusing on microscale power $(<\mathrm{W}$ level, normally $\mathrm{nW}$ to $\mathrm{mW})$ has attracted more and more attention worldwide for the replacement of millions of batteries in portable/wearable electronics and wireless sensor nodes (WSNs), thus realizing self-charging electronics and self-powering smart WSN systems [6-9]. Such energy harvesters scavenge ambient renewable/sustainable energy sources and convert them into electricity by implementing various transduction mechanisms [10-13], such as photovoltaic, thermoelectric, pyroelectric, piezoelectric, electromagnetic, triboelectric, and so on, providing sustainable power solutions.

Until recently, energy harvesters have normally been designed to use a single energy source. For instance, photovoltaic harvesters are developed for harvesting light/solar energy; thermoelectric and pyroelectric harvesters are specially designed for harvesting thermal gradients or fluctuations; piezoelectric, electromagnetic, triboelectric and electrostatic harvesters are particularly for kinetic energy scavenging. However, most of the renewable/sustainable energy sources in our ambient environment may not always be stable or available. Since the energy generation of a single harvester is subject to the availability of the energy source, it cannot always fulfill the power requirement of the electronic devices. For example, solar energy has become one of the most commercial renewable energy. However, photovoltaic harvesters cannot work well at night, on rainy days or in dark environments. 
Kinetic energy is ubiquitous in the ambient environment, including human activities, structural and machinery vibrations, wind flows, water waves, etc. However, the kinetic energy may be fluctuating and insufficient, since a person needs to rest, a machine may not operate continuously, and wind flow or water waves may not occur all the time. In these circumstances, kinetic energy harvesters will be ineffective. Similar situations would also be exhibited in thermal energy harvesters, when there are unpredictable temperature fluctuations/gradients.

To conquer the energy-insufficiency issue of a single energy harvester, hybrid energy harvesting technology is becoming an emerging consensus. Broadly speaking, it represents not only scavenging energy from multiple sources, but also converting energy into electricity by multiple types of transduction mechanisms, where hybrid materials, structures and mechanisms are capable of improving the energy conversion efficiency and/or benefit from different energy sources simultaneously. As a result, hybrid energy harvesters can be divided into two categories: multi-source hybrid harvesters, and single-source harvesters with hybrid mechanisms. In general, multiple energy sources coexist simultaneously or alternately. For example, the vibration energy of a machine or a human body is often accompanied by the generation of thermal energy. The generation of ocean wave energy is mainly because of the existence of wind energy. Obviously, a single-source energy harvester is not capable of scavenging all the coexisting energy, and hence a large amount of available energy is wasted. Multi-source hybrid energy harvesters with diverse energy conversion materials and configurations have been consequently developed as integrated devices $[14,15]$. The output power can be significantly improved when multiple energy sources are available simultaneously or alternately. Apart from the multi-source hybrid energy harvesters, the discovery and development of high-efficiency single-source harvesters with various energy transduction mechanisms still occupies the mainstream research direction. A number of technical reviews on piezoelectric [16-19], electromagnetic [20], triboelectric [21], thermoelectric [22], pyroelectric [23] or hybrid mechanisms [24, 25], have been presented, focusing on materials [26, 27], theories [28], configurations, and applications. Recently, emerging energy harvesting principles have seen 
reported, such as flexible organic ionic diodes [29], flexoelectric [30], ferroelectret [31], electrochemical [32], mechanoradical [33] and biomaterial-based [34] mechanics, and others. Through the comparative study of the presented energy harvesters, it can be found that different harvesting materials and mechanisms may be applicable to different structural configurations and application scenarios. A reasonable combination of two or more separated harvesting materials or mechanisms provides not only improvement of the space utilization efficiency, but also significant increase of the power output. For example, piezoelectric, electromagnetic, and triboelectric harvesters are considered best suited to scavenge energy from kinetic vibrations. A vibration-based energy harvester normally employs a spring-mass structural configuration, where the suspension structures can be integrated with piezoelectric material, while the proof mass may be used as the magnet of the electromagnetic harvester or integrated with triboelectric material in addition to its function of adjusting the resonant frequency of the system. Hence, the output power could be a superposition of individual piezoelectric, electromagnetic, or triboelectric harvesters without additionally increasing much volume and complexity. Therefore, the use of hybrid transduction mechanisms or materials with an optimized configuration is more desirable in terms of the power generation efficiency.

Besides integrating the transduction mechanisms, effective hybrid systems require appropriate power integration circuits, in order to deliver to the load as close as possible to the sum of the individual harvester output power levels. However, it is worth noting that in some cases one of the two mechanisms produces less more power than the other, making the additional device size and complexity required to add this mechanism unjustified by the added power. Thus, one measure of the value of hybrid devices is whether each mechanism makes a substantial power contribution or provides some other vital function such as a bias voltage for another mechanism.

Many researchers have presented effective and innovative strategies on hybrid energy harvesters from single-source/multi-source in the past few years [35-38]. However, these works 
have not been systematically studied and summarized, because the number of publications is relatively small compared with the single-source harvesters. Followed by the continuously growing number, we herein give a comprehensive review and present recent progress in the hybridization of energy harvesting systems, especially focusing on vibrational and thermal types. These types of hybrid energy harvesters are the most investigated areas due to the availability of vibrational and thermal energy and the diversity of relevant conversion mechanisms. The rest of this review is organized as follows. Firstly, the background and operating principle of some common energy conversion mechanisms, including piezoelectric, electromagnetic, triboelectric, thermoelectric, and pyroelectric, are briefly introduced, and categorized in Section II. Thereafter, the typical hybrid energy harvesters including the mechanisms, configurations, output performance and advantages are elaborated in Section III. On this basis, performance comparisons and discussions are presented in Section IV. Simple but straightforward perspectives are selected to evaluate the performance and effectiveness of the hybrid vibrational and thermal harvesters. Finally, an overview of the diversity of the potential applications is presented, including the available energy sources for the hybrid integrated system to be a self-powered sensor node or power source.

\section{Energy Conversion Mechanisms}

\section{A. Piezoelectric effect}

Piezoelectric effect was first discovered by the brothers Pierre and Jacques Curie. It can be divided into direct piezoelectric effect and converse piezoelectric effect. The former is defined as the phenomenon where electric potential is induced across a material when it is strained under an applied force, while the latter is conversely defined as the phenomenon where mechanical strain is generated in a material when it is exposed to an electrical field. The direct and converse piezoelectric effects can be represented by the piezoelectric constitutive equations as: 


$$
\left[\begin{array}{l}
\delta \\
D
\end{array}\right]=\left[\begin{array}{ll}
S^{E} & d^{t} \\
d & \varepsilon^{T}
\end{array}\right]\left[\begin{array}{l}
\sigma \\
E
\end{array}\right]
$$

where $\sigma$ and $\delta$ are the stress and strain components; $E$ and $D$ are the electric field and electric displacement, respectively; $s, \varepsilon$ and $d$ stand for the elastic compliance, dielectric constant and piezoelectric coefficient, respectively; the superscripts $E$ and $T$ denote the evaluated constants under constant electric field and constant stress, respectively; and the superscript $t$ indicates the transpose [39].

Typically, a piezoelectric energy harvester (PEH) uses a resonant-based configuration as shown in Fig. 1(a-i). A cantilever with bimorph/unimorph piezoelectric material, such as lead zirconate titanate (PZT), is one of the widely used structures in the resonant mode. As the cantilever oscillates under the excitation of external force, the piezoelectric layer deforms to generate electric potential accordingly. In order to increase output performance, many other beam shapes, besides conventional rectangular cantilevers [40-42], have also been investigated, including triangular [43], taper [44], S-shape [45], arc-shaped [46], as shown in Fig. 2 (a)-(e). In addition to the above bending or tensile mode configurations, flexure stress amplification configurations are also preferred due to the superior compressive strength of piezoceramics. Fig.1(a-ii) also shows a cymbal-like PEH which consists of a piezoelectric stack sandwiched by the top and bottom metal end caps [47]. The end caps amplify the stress and turn it into a longitudinal stress in the PZT stack. Fig. 2(f) shows a high-efficiency compressive-mode energy harvester employing a pair of elastic beams and a flex-compressive center where the piezoelectric element bears a dynamic compressive load. It is beneficial to an enlarged piezoelectric power generation process [48].

\section{B. Electromagnetic effect}

Michael Faraday discovered the law of electromagnetic induction, which not only revealed the internal connection between electricity and magnetism, but also laid the experimental foundation for the mutual conversion between them. Based on Faraday's law of electromagnetic 
induction, when the magnetic flux passing through a closed coil changes, electric current will be induced. The corresponding induced voltage $E$ is given by:

$$
E(t)=-N \cdot \frac{d \Phi}{d t}
$$

where $N$ is the number of coil turns, $\Phi$ is the magnetic flux, and $t$ is the time [49].

Various innovative configurations based on electromagnetic effect have been developed to date, such as resonant structures in approaching-separation mode (ASM) [50, 51], and the rotational structures in relative-sliding mode (RSM) [52]. Fig. 1(b-i) shows a simplified diagram of the traditional resonant electromagnetic energy harvester (EMEH) in ASM. A magnet mounted on a planar spring vibrates relative to the stationary coils under the excitation of external vibration, which changes the magnetic flux in the coils and thus generates induced voltage. For the purpose of obtaining resonant vibration, the spring structure can be a spiral cantilever, a freestanding or fixed-fixed beam structure as illustrated in Fig. 3(a)-(c) [50, 53, 54]. The Halbach array is a method to enhance the obtainable magnetic flux density by arranging the magnets in a special manner. Some example work can be found in Refs $[55,56]$. An example illustration is shown in Fig. 3(d) [56]. As shown in Fig. 1(b-ii), a rotational EMEH in RSM mainly consists of rotor magnets and stator coils, or vice versa. Supported by conventional roller bearings, the rotor magnets can rotate with the driving shaft, which generates a time-varying magnetic flux in the stator coils and hence induced voltage in the coils. With respect to the rotatory motion, the rotor magnets or coils can be installed on a pendulum structure, an eccentric structure, or a rotatory mass (Fig. 3(e)-(g)) and so on [57-60].

\section{Triboelectric effect}

The triboelectric phenomenon has been probably known for a long history, but it is typically considered as a negative effect causing unexpected consequences e.g. in modern factories. Since Z L Wang's research group first reported the triboelectric nanogenerator (TENG) in 2012, an increasing number of TENGs have been presented. Triboelectric effect is considered 
as the coupling effect of triboelectric and electrostatic induction produced by contact-separation or relative-sliding between two materials with opposite friction polarity, such as polytetrafluoroethylene (PTFE) and aluminum (Al). If the electric potential of the bottom electrode is defined as zero, the electric potential of the top electrode $U$ can be expressed as

$$
U=-\frac{\sigma \mathrm{d}}{\varepsilon}
$$

where $\sigma$ is the triboelectric charge density, $d$ is the interlayer distance at a given condition, and $\varepsilon$ is the vacuum permittivity, [61].

Based on different electrode configurations and working modes of triboelectric layers, there are four fundamental types of TENGs in Fig.1(c): contact-separation mode (CSM), lateralsliding mode (LSM), single-electrode mode (SEM), and freestanding triboelectric-layer mode (FTM). The latter two modes are extensions of the former. (i) In the contact-separation TENG, there's a small gap vertically between two triboelectric films with a large difference in electron negativity facing opposite to each other. By applying an external force, the gap is gradually reduced to zero and the two triboelectric films come into contact with each other, which generates electrically opposite charges on each contact surface. As the two surfaces contact/separate in the vertical direction, an electric potential will be reduced/generated across the two electrodes, making the electrons flow back and forth via an external circuit. In order to achieve the CSM, several configurations have been presented, such as the spring-assisted structure [62] in Fig. 4(a), the arc-shaped structure [63] in Fig. 4(b) and so on. (ii) The lateralsliding TENG shares the same starting position with the contact-separation one. Triboelectric charge is produced by sliding on two triboelectric surfaces. As can be seen from Fig. 1(c-ii), when the triboelectric layers in contact with each other begin to slide relatively, electric charge and potential difference are generated along the sliding direction, which drives the electrons on the top and bottom electrodes to flow in the external circuit to balance the potential difference generated by triboelectric charge. The lateral-sliding TENGs can be further divided into planar motion type [64], disc rotation type [65], and cylindrical rotation type [66], as illustrated in Fig. 
4(c)-(e), according to their structural characteristics. It is noteworthy that the output performance of TENGs could be greatly enhanced through making parallel or radial grid electrodes in the LSM devices. (iii) Besides the aforementioned dual-electrode modes, the single-electrode TENGs have also attracted considerable attention, where the other electrode is connected to ground. Within a finite range of dimensions, the electric field distribution is bound to change when the top triboelectric layer is approaching to or departing from the bottom electrode. As a result, charge transfers back and forth between the bottom electrode and the ground so as to keep the potential balanced. The relative motion between the triboelectric layer and the electrode can be vertically contact-separation [67] or lateral-sliding [68, 69], shown in Fig. 4(f)-(g). (iv) The freestanding TENG is mainly composed of two stationary coplanar electrodes with a symmetric configuration and a free triboelectric layer without electrodes. The reciprocating motion of a charged object between two electrodes causes a change in the potential difference, which in turn drives the electrons to flow back and forth between the two electrodes through an external circuit to keep the potential balanced. The freestanding triboelectric-layer TENGs also have two typical configurations: contact-separation [70] and lateral sliding [71], as shown in Fig. 4(h)-(i).

\section{Thermoelectric effect}

Thermal energy is abundant in many domestic and industrial applications, such as engines, machines, and human bodies. Therefore, effective thermoelectric energy harvesters (TEH) are indispensable for the realization of energy sustainability. Thermoelectric energy harvesting has been extensively investigated as one of the promising mechanisms for energy conversion [7274]. As thermoelectric devices use the temperature difference between two surfaces, no moving parts are required in such generators, which allows them to be reliable, easy-to-implement and scalable.

The Seebeck effect, discovered by T. J. Seebeck in 1821 [75], paved the foundation of TEH and is still the most widely used phenomenon in thermoelectric generation or cooling due to its 
high output power and material versatility. The Seebeck effect is a phenomenon in which a voltage difference between two distinct electrical conductors or semiconductors is produced due to a temperature difference being applied, as illustrated in Fig.1(d). In thermoelectric devices, segments of p- and n-type doped materials, (e.g. appropriately doped bismuth telluride), are combined in a ' $\Pi$ ' structure (junctions of two materials with different Seebeck coefficients) to enhance the output power for a given temperature difference. In practice, TEHs consist of large numbers of junctions electrically in series and thermally in parallel, to enhance output voltage and spread heat flow, as shown in Fig.1(d-i). The efficiency of a TEH is characterized using the dimensionless figure of merit $Z_{S E} T$, defined as

$$
Z_{S E} T=\frac{\alpha^{2}}{\kappa \rho} T
$$

where $\alpha, T, \kappa$ and $\rho$ are the Seebeck coefficient, average absolute temperature of the thermoelectric materials, thermal conductivity and electrical resistivity [76]. The coefficient $\alpha^{2} / \rho$ is defined as the thermoelectric power factor. The theoretical maximum efficiency of a TEH, i.e. $\eta_{T E H}$, can be written as a function of $Z_{S E} T$ and the temperature gradient $\Delta T=T_{h}-$ $T_{c}$ between two surfaces $\left(T_{h}\right.$ and cold $\left.T_{c}\right)$, as

$$
\eta_{T E H}(\Delta T)=\frac{\Delta T}{T_{h}} \cdot \frac{\sqrt{1+Z_{S E} T}-1}{\sqrt{1+Z_{S E} T}+T_{c} / T_{h}}
$$

The output electrical power is then the heat flux times this efficiency. For telluride alloy TEHs, the $Z_{S E} T$ value is about 1 . When operating at $\Delta T=20 \mathrm{~K}$ and close to room temperature, the theoretical efficiency is around $1 \%$. This illustrates the intrinsic low conversion efficiency of the harvester and the dependence of the operating condition. Many efforts have been dedicated to improving the figure of merit of thermoelectric materials [77, 78]. For sufficiently high figure of merit, the ultimate limit on $\eta_{\text {TEH }}$ is the Carnot efficiency, $\Delta \mathrm{T} / \mathrm{T}_{\mathrm{h}}$. It should be noted that enhancing heat flux is as important to maximizing electrical power as increasing $\eta_{\text {TEH. }}$

In terms of application, TEHs are typically used in conditions where a distinct temperature difference is available or ambient temperature undergoes regular variation. In the first case, 
devices are designed for waste heat recovery [79], harvesting human body heat for wearable sensors [80, 81], solar-driven thermoelectric generator [82-84], nuclear-powered TEHs in space [85], etc. Devices shown in Fig. 5 are typical structures for applications. Various considerations have driven research in TEHs, such as effective power management, wearability and flexibility. A high-performance flexible TEH was developed using screen printing as well as a laser multiscanning lift-off process, as shown in Fig. 5(b) [86]. Output of $4.8 \mathrm{~mW} / \mathrm{cm}^{2}$ at $\Delta T=25^{\circ}$ was obtained, and the devices had no significant performance degradation under 8000 cycles repeated bending.

In cases where the ambient temperature changes regularly, phase change materials (PCM) can be integrated with TEHs to create a spatial temperature difference. An example application in aircraft condition monitoring is illustrated in Fig.5(d)-(e), where a thermoelectric panel was combined with a container with phase change materials to harness the temperature variation during aircraft landing and take-off [87-89]. The temperature gradient between the PCM and the environment provides the energy source for the TEH. Similar devices and ideas have also been used in solar-driven TEH [90], self-powered environmental monitoring [91] and thermoelectric cooling [92].

\section{E. Pyroelectric effect}

While thermoelectric materials can convert temperature gradients into electrical power, the pyroelectric effect generate electricity from temperature fluctuations [93]. Pyroelectric materials are polarized and exhibit a spontaneous polarization $P_{s}$ without of an electric field. Pyroelectric behaviors originate from the polarization level which is governed by material temperature [94]. If the temperature increases $(\mathrm{d} T / \mathrm{d} t>0)$, the polarization level reduces, because the dipoles' orientation is lost, and the amount of unbound charge on the surface reduces; electric currents are generated as a result. If the temperature is reduced $(\mathrm{d} T / \mathrm{d} t<0)$, the dipoles re-orientate, and a reverse current flow is produced, as shown in Fig.1(e) [95]. The governing equation between the generated current $\left(i_{p}\right)$, and the temperature gradient $(\mathrm{d} T / \mathrm{d} t)$ is 


$$
i_{p}=\frac{\mathrm{d} Q}{\mathrm{~d} t}=A \cdot p \cdot \frac{\mathrm{d} T}{\mathrm{~d} t}
$$

where $A$ is the material surface area and $p$ is the pyroelectric coefficient, which can be written as

$$
p=\frac{\mathrm{d} P_{S}}{\mathrm{~d} T}
$$

where $P_{S}$ is spontaneous polarization [96]. Pyroelectric harvesters are usually modelled as current sources. The net charge generated due to a temperature variation $\Delta T$ is

$$
Q=p A \Delta T
$$

Since pyroelectric materials are commonly dielectric, their internal capacitance $(C)$ can be represented as

$$
C=\frac{A \varepsilon_{33}^{\sigma}}{h}
$$

where $\varepsilon_{33}^{\sigma}$ is the material permittivity in the polarization direction. The generated electrical energy in a capacitor is $Q^{2} /(2 C)$. Therefore, the stored energy due to temperature variation is

$$
E=\frac{1}{2} \frac{p^{2}}{\varepsilon_{33}^{\sigma}} A h(\Delta T)^{2}
$$

Pyroelectric materials generally do not need bulky heat sinks to generate a large temperature gradient due to their high conversion efficiency [97]. However, this effect requires temperature oscillation, which poses challenges in generating such a fluctuation. Natural temperature fluctuations are rare but exist in conditions with variable convection conditions [98], light irradiance variation [99, 100] or exhaust gas [101-103], as shown in Fig. 6(b) and (c). Electronics operating and switching between different modes can also provide temperature fluctuations [104]. The correlated challenge is the low-frequency feature of many such temperature oscillations, which would limit the energy generation performance compared to vibration energy harvesters. Mechanisms to increase the frequency of temperature oscillation would provide solutions, such as using oscillating heat sources [105], bi-metal materials [106] or liquid-based switchable interfaces [97, 107, 108], as shown in Fig.6(a), (d) and (e). 


\section{Hybrid Energy Harvesting Systems}

In the previous section, the operating principle for each conversion mechanism has been discussed. The integration of multiple conversion mechanisms in one design, as hybrid energy harvesters, is discussed in this section to investigate the necessity and benefits of such integration and to identify the current barriers and future research directions.

\section{A. Piezoelectric-electromagnetic (PE-EM) hybrid systems}

Piezoelectric and electromagnetic conversion mechanisms are commonly used to generate electricity from kinetic energy. The integration of these mechanisms has been the first attempt in hybrid energy harvesting to enhance energy generation capability and system power density. Enhancing and matching the electrical damping to the mechanical one is one of the intentions of such solutions to enhance the energy conversion efficiency in hybrid energy harvesters. Toyabur et al. developed a multimodal piezoelectric-electromagnetic hybrid harvester to harness broadband vibration energy sources, as shown in Fig.7(a) [109]. An improvement in output power was obtained using this hybrid method without significant increase in device size. Yang et al. developed a similar PE-EM hybrid harvester based on a cantilever beam structure; further efforts were made on developing the micro-machined coil design and fabrication to reduce added size and weight, as shown in Fig.7(b) [110]. To optimize the performance of such hybrid harvesters, Xia et al. studied the dynamics of such a hybrid topology in a dimensionless manner for different electrical boundary conditions, further illustrating the capability of hybrid energy harvesting in enhancing the output power and conversion efficiency. [111]. Li et al. studied the PE-EM hybrid harvester on a fixed-fixed beam design with a magnet proof mass in the beam center. The coupling effect was theoretically investigated, concluding that hybrid energy harvesting should be used with weak and medium coupling (electrical damping ratio < $2 \times$ mechanical damping ratio) [112].

In order to enhance the operational bandwidth, Mahmoudi et al. introduced nonlinear dynamics (mono-stable) into a hybrid harvester by applying repulsive magnetic forces on a 
magnet in the center of a fixed-fixed beam [113]. Enhancement in power density, bandwidth and output power was obtained in the numerical study. Xia et al. developed a novel method for the typical PE-EM harvesters by adjusting the axial magnetic force, as shown in Fig. 7(d) [114]. A wide operation range from $26 \mathrm{~Hz}$ to $62 \mathrm{~Hz}$ was obtained. Multi-mode vibration can also provide enhanced bandwidth. Inspired by this, Xu et al. introduced a second magnetic oscillator between the tip magnet and coils in the cantilever-based harvester, as shown in Fig. 7(e) [115]. Hamid and Yuce developed a PE-EM harvester for wearable devices with the study of appropriate power management circuits [116]. Recently, Fan et al. developed a nonlinear hybrid energy harvester using a sliding magnet supported by repulsive forces in a tube with two fixed magnets on each end to excite a set of coils and two piezoelectric beams, as shown in Fig. 7(c) [117]. The harvester exhibited high energy generation capability for ultra-low frequency energy sources, such as handshaking. Li et al. developed a $0.33 \mathrm{~W}$ PE-EM hybrid energy harvester using a truss mechanism and a mechanical stopper to amplify the motion and to introduce frequency up-conversion and non-linearity into the system, as shown in Fig. 7(f) [118]. With the overall dimensions of $42 \times 58 \times 54 \mathrm{~mm}$, the harvester generated $0.33 \mathrm{~W}$ peak power under the acceleration of $0.7 \mathrm{~g}$ at $6.9 \mathrm{~Hz}$. Yang and Cao presented a novel tri-stable hybrid harvester design to address the challenge of harnessing ultra-low level vibration energy sources, as shown in Fig.7(g) [119]. It is worth mentioning that the focus on nonlinear dynamics is more on broadening the operational bandwidth as in non-hybrid energy harvesters, rather than the synergy of piezoelectric and electromagnetic mechanisms; the lack of effective integration and synergy of different mechanism is also one of the common issues and shortcomings of most hybrid energy harvester works in the literature.

In addition to vibration energy, efforts have also been made in converting other types of kinetic energy sources, such as airflow or sound, using hybrid energy harvesters. Dias et al. adopted a cantilever-based hybrid harvester to capture airflow energy by attaching an airfoil at the beam's free end, as shown in Fig.8(a) [120]. Khan et al. developed a PE-EM harvester for acoustic energy harvesting, in which a Helmholtz resonator, a PE host with two permanent 
magnets, and a set of coils located underneath the magnets were designed to convert the acoustic-induced vibration on the resonator into electricity, as shown in Fig.8(c) [121]. Iqbal and Khan combined the piezoelectric and electromagnetic conversions in a hybrid airflow and vibration energy harvester with an airfoil and tip magnet attached on a cantilever beam's free end for bridge health monitoring applications (Fig.8(b)) [122]. A second adjustable beam was introduced to produce multi-mode vibrations that increase the operational bandwidth. Zhao et $a l$. developed a water-proof PE-EM hybrid wind harvester by adopting a micro-turbine with reduced magnetic resistance torque, as shown in Fig.8(d) [123].

Power management circuit design is another critical challenge for hybrid energy harvesting. Outputs in alternating current form are typical for piezoelectric and electromagnetic harvesters. Rectification, energy storage and voltage stabilization are necessary to accumulate collected charges on a single storage. As discussed earlier, PEHs typically exhibit high output impedance due to low capacitance and operating frequency. High output voltage and low output current are the characteristics of PEHs because of this high output impedance. However, EMEHs generate high output current and low voltage due to the low impedance of in the coils. The distinct output differences between PEHs and EMEHs result in great challenges for effective interface design for hybrid harvesters. The straightforward solution is to design rectification and storage units for the piezoelectric and electromagnetic harvesters individually and to drive a load simultaneously, as shown in Fig.9(a) [124]. A detailed design in how to implement such a power management system and the potential mechanisms to enhance the efficiency were presented in [125], as shown in Fig.9(b). However, the separate interfaces limit the efficiency of the power management circuits. To tackle this issue, Chamanian et al. developed an efficient interface of hybrid harvesters to synergistically manage power from EM and PE sources [126]. The functional blocks and operation mechanism are depicted in Fig.9(c) and (d). Compared to conventional systems, the functions of energy integration and extraction are synchronized and implemented at the harvester interface. Synchronous energy integration was obtained through what the authors call an "investment time manager". This unit extracts charge from the EM 
harvester and injects it synchronously into the PEH to increase the damping force, thus realizing the high efficiency synchronous electric charge extraction (SECE) without the need for an external power supply.

Other interface solutions for multiple energy sources, including vibration, thermal and light, have also been investigated to enhance the energy harvesting conversion efficiency [127, 128]. However, compared to the mechanical design of hybrid energy harvesters, power management circuit design for hybrid harvesters is still under-developed. Interfaces that can be adapted to different specific operating frequencies with improved conversion efficiency over a large amplitude range are one necessary development for the advancement of hybrid PE-EM energy harvesters.

PE-EM hybrid energy harvesters have been investigated extensively in terms of structural design, but lack of synergy to amplify the advantages of both piezoelectric and electromagnetic conversion in one hybrid design is one of the issues. Most of the current publications are either on adding two conversions independently or on studying other nonlinear dynamics which could also work in a non-hybrid system. Power management circuits are the second key issue due to the significant difference between PEH and EMEH. More effective solutions to manage two power sources effectively are of great importance to make PE-EM hybrid harvesters more advantageous.

\section{B. Piezoelectric-triboelectric (PE-TE) hybrid systems}

The piezoelectric and triboelectric effects are two different mechanical-to-electrical energy conversion approaches. Under an external mechanical force, the TENG produces a charge potential difference on the material surfaces during contact and separation processes of the two triboelectric materials, whereas the $\mathrm{PEH}$ generates internal potential difference across the piezoelectric material during stretching and compressing processes. On this basis, these two effects present some common operational characteristics in response to mechanical vibration, deflection and compression, which can be further combined as a hybrid energy harvesting 
system to enhance energy output. Some promising designs have been illustrated by integrating the piezoelectric and triboelectric mechanisms with separated or shared material components.

When an external force is applied to an appropriate configuration, such as arc-shape, $r$ shape, parallel-plate, etc., both the deformation and contact friction of the materials will occur at the same time as shown in Fig.10(a). Therefore, it is straightforward to achieve cooperative operation of piezoelectric and triboelectric materials in the form of vertically stacked layers by a single press-and-release process. Jung et al. [129] presented an arc-shaped PE-TE hybrid generator using $\mathrm{Au} /$ Polyvinylidene difluoride (PVDF) /Au sandwiched layers on the top as the $\mathrm{PEH}$, and PTFE/Al layers on the bottom as the contact-separation type TENG, where the organic ferroelectric PVDF is the piezoelectric material, and the common electrode Au is shared as the bottom electrode of the PEH and a driving electrode of the TENG. When an external force is applied, the PVDF gains a tensile stress and generates a positive piezoelectric potential, and electrons flow between the sandwiched electrodes. In the meantime, the friction between the common Au electrode and the bottom PTFE layer is induced, and a charge transfer occurs since the PTFE tends to gain electrons with respect to the Au due to triboelectric effect. Similar PE-TE hybrid systems have been reported by a few groups [130-133] with varied contact-andseparation configurations and functional materials. Overall, the piezoelectricity can be generated by deformation of the piezoelectric material itself during a press-and-release process, and meanwhile can be enhanced from the electric charges generated by triboelectric effect.

Recently, some pioneering works have shown that the piezoelectric and triboelectric effects can coexist in one functional material and interact on each other, which opens a new way of improving the performance hybrid PE-TE devices. Suo et al. [134] mixed ferroelectric barium titanate (BTO) nanoparticles (NPs) into a polydimethylsiloxane (PDMS) polymer matrix, forming a BTO/PDMS composite film, which generated higher performance than a pure PDMS film. It is observed that the addition of BTO NPs with positive polarization will enhance the contribution of piezoelectricity. On the contrary, the reverse polarization reduces voltage output of the composite film because of the mutual cancellation of piezoelectricity and triboelectricity. 
Thereafter, functional materials with both piezoelectric and triboelectric effects have been reported regularly. Karumuthil et al. [135] prepared composite films using zinc oxide ( $\mathrm{ZnO})$, exfoliated graphene oxide (EGO) and multiwalled carbon nanotubes (MWCNTs) in PDMS along with a hybrid operation mechanism based on both PE and TE characteristics. Qian et al. [136] reported a PDMS/ZnO nanoflakes/3D Gr heterostructure-based hybrid harvester (in Fig.10(b)), simultaneously exploiting the piezoelectric effect and triboelectrification, resulting in an ultrahigh power density of $6.22 \mathrm{~mW} / \mathrm{cm}^{2}$. Chowdhury et al. [137] reported a piezoelectric PVDF film mixed with ZnO nanowires (NWs) and MWCNT, while the PDMS-PTFE copolymers is acted as a triboelectric layer on thin Al film. Guo et al. [138] electrospun silk fibroin and PVDF nanofibers on conductive fabric to fabricate piezoelectric reinforced TENG composite film. The device acted as a self-powered cloth-like sensor and was integrated with the wearers' clothes. These works indicated that the triboelectric and piezoelectric effect can be simultaneously induced through the modification of a composite film.

The focus of the above works is mainly on improving the cooperativity of the piezoelectric and triboelectric materials. They did not focus on the utilization of the external kinetic energy sources in the form of vibrations, wind flows or water waves, etc. It is known that a resonantbased spring-mass configuration is normally employed to harvest vibration energy with high efficiency at the condition when the resonant frequency is matched with the environmental vibrations. Therefore, additional requirements of low operating frequency and wide bandwidth are preferred. By integrating piezoelectric and triboelectric materials on a structure, piezoelectricity and triboelectricity can be generated by a single tensile-compressive process of the piezoelectric material and contact-separation process of the triboelectric material in response to an external vibration. Han et al. [139] presented a low-frequency and wideband hybrid harvester, which produce both piezoelectric and triboelectric outputs by the use of an array of piezoelectric PVDF cantilevers periodical impacting with the bottom triboelectric PDMS film. Similarly, Singh and Khare developed an impact-based PE-TE harvester using PVDF and Zno, as shown in Fig.11(a) [140]. The shared Al electrode acts not only as a bottom 
electrode of the piezoelectric layer but also as a driving electrode of the triboelectric layer. $\mathrm{Li}$ et al. [141] made use of the impacts via an amplitude stopper with TENG patch, and the force magnification effect of a truss mechanism with a PEH patch to achieve larger voltage and wide bandwidth (in Fig.11(b)). There are also some wind harvesters that implement piezoelectric and triboelectric effects simultaneously. Chen et al. [142] proposed a hybrid wind energy harvester based on the vortex shedding effect (in Fig.11(c)). The specific design of flapping blade and spindle-shaped frames is able to boost the output of a PVDF cantilever and the triboelectric effect between the PDMS on the inner surface of the frames and the $\mathrm{Al}$ on the flexible cantilever. In 2019, Z. L. Wang's group introduced a hybrid PE-TE rotational wind harvester by integrating a bimorph-based PEH into a one-end-fixed TENG [143]. As shown in Fig.11(d), at the bottom of the rotor shaft there is a polyethylene terephthalate (PET) film rotating with the rotating shaft, and the bottom of the device uniformly distributes three sets of piezoelectric and triboelectric composite transducer sheets along the circumference. Driven by the top wind cup, the PET film sequentially slides the surrounding composite transducer sheet to generate piezoelectric and triboelectric signals.

\section{Electromagnetic-triboelectric (EM-TE) hybrid systems}

Typically, the relative movement of the magnet and coil in an EMEH can be realized by a number of structures, but these fall into three main categories, i.e. resonant, nonresonant, and rotational structures. During the movement of the structures, the TENG can be integrated and actuated to scavenge energy from the contact-separation and sliding friction between two triboelectric materials. By combining these two energy harvesting schema, more electricity can potentially be generated from one mechanical movement. It is also noted that the difference of their output characteristics affects the electric charging characteristics. In cases where harvesters are used to charge a capacitor as an energy store, the output voltage of the harvester determines the maximum charge level of a capacitor (in the absence of a voltage converting interface circuit), while the charging speed is determined by the generated current. Because of 
the high voltage and low current outputs of the TENG, a capacitor can be charged directly up to tens or hundreds of volts, although typically at the cost of a long charging time. On the other hand, the EMEH generates low voltage (often several volts) but high current, so a capacitor can be charged quickly but reaches saturation within a short charging time [144, 145]. Therefore, some researchers have sought to combine TENG and EMEH in one hybrid device, to gain the advantages of both. However, in a number of application scenarios, the EMEH and TENG cannot easily share power processing circuits. The other important issues to consider are the efficiency of rectification from typically AC harvester outputs to the DC needed for charging capacitors or batteries, the impedance match between the circuit load and the EMEH or TENG, as well as the effects of parasitic capacitances; all these make combining the outputs more complex.

For the resonant-based structures, there are a variety of implementation mechanisms to form the EM-TE hybrid system, such as spring-mass, deflected-membrane, and magneticfloating structures. As shown in Fig.12(a) and (b), two similar broadband EM-TE hybrid harvesters have been reported by using a magnetic proof mass connected with a polymer spring [146] and a copper spring [147], separately. The electromagnetic coils are located on the top or surrounding the movable magnet. The two triboelectrically opposite materials (PTFE/IndiumTin-Oxide (ITO) and Fluorinated ethylene propylene (FEP)/Cu) are integrated with the movable magnet and the top or bottom base substrate, respectively. As the spring structure together with the magnetic mass is oscillated, the change of the magnetic flux across the coil will be introduced. In the meantime, the triboelectric film covered on the magnet surface will impact with the bottom triboelectric layer periodically. Therefore, in one complete oscillation, the device generates electromagnetic and triboelectric outputs, simultaneously, which acquires not only higher energy conversion efficiency but also broadening of the operating bandwidth. In Fig.12(c), Chen et al. [148] presented a hybrid harvester using a freestanding magnet and spring structure, which is able to harvest in-plane vibration from arbitrary directions by the EMEH. Besides, it can detect the vibration direction from periodical or pulse movement based on the 
eight-petal electrodes of the TENG. In addition to the direct use of the spring structure, there are some schemes that adopt deflected-membrane structures [149]. In Fig.12(d), Wang et al. [150] developed a hybrid harvester integrating an EMEH and a TENG for harvesting wind energy simultaneously. As the middle vibrating FEP film is moved up and down by wind flow, it will contact with the up and bottom electrodes, resulting in electron flow from the TENG part. Meanwhile, due to the distance change between the vibrating magnets on the middle film and the coils on the up and bottom bases, the EMEH parts can deliver output current/voltage, simultaneously.

In order to efficiently harvest low frequency human motion energy, Seol et al. [151] employed a magnetic-floating structure, which was embedded into an EM-TE hybrid system, as shown in Fig.12(e). The oscillating magnet is suspended in the tube by dual-directional magnetic repulsive forces. The change of magnetic flux of the floating magnet induces an electrical current in the surrounding coils of the EMEH part. Meanwhile, the sliding friction between the magnet and sidewall induces contact electrification in the TENG part. By employing a similar magnetic-floating structure, Salauddin et al. $[152,153]$ designed a Halbach-array magnetic frame as the floating oscillator, as shown in Fig.12(f). A bobbin wound with copper coil was positioned inside the hollow oscillator to form the EMEH component. The $\mathrm{Al}$ electrode was attached to the outside surface of the oscillator frame, and the microstructured PTFE film was attached to the inside sidewall of the housing. The TENG component delivers an output signal during the sliding friction of the two triboelectric materials. The fabricated hybrid EM-TE harvester exhibited much higher power output than similar reported works.

The above mentioned resonant structures tend to be ineffective for harvesting low frequency and random vibrations, such as human motion or water waves. Therefore, rolling cylinders or rolling balls have been widely adopted to form the non-resonant structures [154]. In Fig.13(a), Wang et al. [155] proposed a water-wave energy harvesting device integrated with TENG and EMEH components. The TENG component consists of a set of Al rolling rods and PTFE film coated with copper interdigital electrodes. In the EMEH component, the motion of 
copper coil array was guided by four steel rods in between the top and bottom magnet array. The hybrid generator can maximize energy conversion efficiency at quite low frequencies of less than $1.8 \mathrm{~Hz}$ and broaden the operating frequency, simultaneously. Recently, Wang et al. [156] and Hao et al. [157] have both employed the rolling of a magnetic cylinder on a platform with interdigital electrodes and coils to form a two-dimensional and ultra-low frequency waterwave energy harvester, as illustrated in Fig.13(b) and (c). The unique magnetic cylinder design enabled the TENG and EMEH easily to be driven and adapted well to the randomness of water waves. In order to power wearable electronic devices in health monitoring, Maharjan et al. [158, 159] presented a wearable hybrid EM-TE harvesting wristband from low-frequency human wrist-motion (Fig.13(d)). The device employed a freely moving magnetic ball inside a hollow circular tube. The total output performance was greatly improved by the fabrication of microstructures on the magnetic ball, nanorods on the tube inner surface and the flux concentrating material integrated with the coil.

Remarkably, there is a considerable number of hybrid harvesters working in rotational mode. Chen et al. [144] and Zhang et al. [160] have demonstrated rotating-disk-based EM-TE hybrid generators for scavenging rotational energy, as shown in Fig.13(e). The rotating structure consisted of a rotor and a stator, so as to induce relative movement between the positive and negative triboelectric materials, as well as the magnets and coils. Previous studies on a winddriven hybrid generator using rotating-sleeve structures have been reported by Qian et al. [161] and Cao et al. [162] (Fig.13(f)). Waterwheel-like hybrid generators have been reported for harvesting rotational energy $[163,164]$. The authors used the integrated cylindrical surface and coupled magnets in rolling cylinders with copper coils to form EMEH and TENG, simultaneously.

\section{Piezoelectric-electromagnetic-triboelectric (PE-EM-TE) hybrid system}

On the basis of the dual-hybrid systems integrating PE-EM, PE-TE, and EM-TE mechanisms, researchers are exploring the possibility of triple-hybrid energy harvesting 
systems. This could be a promising route to further improve output performance by hybridizing the PE-EM-TE harvesting mechanisms in one device.

In Fig.14(a), He et al. [165] investigated a low-frequency PE-EM-TE hybrid broadband vibration energy harvester by using three layers of spring-mass structures. A PVDF film attached with magnet is suspended in the middle layer to generate electricity based on the piezoelectric and electromagnetic mechanisms. The top and bottom suspended PDMS layers are used to generate electricity by periodic contact-separation with the $\mathrm{Cu}$ electrode based on triboelectric effect. At the acceleration of $0.5 \mathrm{~g}$ and vibration frequency of $20 \mathrm{~Hz}$, the maximum power outputs of the PEH, EMEH and TENG are 41, 66.5 and $4.6 \mu \mathrm{W}$ at their matched load resistances of $800 \mathrm{k} \Omega, 343 \Omega$ and $1.4 \mathrm{M} \Omega$, respectively. By hybridizing PE-EM-TE mechanism, the whole device can not only harvest vibration energy over a wide bandwidth, but also achieve enhanced output.

Besides employing conventional mechanical suspension structures like springs or cantilevers, He et al. [166] reported a hybridized PE-EM-TE generator employing a core magnetic floating structure with a higher vibration sensitivity (Fig.14(b)). Below $20 \mathrm{~Hz}$, the bottom and top EMEH produced a peak power of 38 and $36 \mathrm{~mW}$, respectively, while the bottom and top PEH generated a peak power of 105 and $122 \mathrm{~mW}$, respectively. The TENG delivered an insignificant peak power of $78 \mu \mathrm{W}$ compared to the other two components.

In addition to collecting vibration energy by resonant suspension structures, Koh et al. [167] demonstrated a self-powered inertial sensor with non-resonant magnetic rolling balls inside a hollow shell, as shown in Fig.14(c). The inner surface of the shell was coated with multilayers of PTFE, PVDF and Al films and the outside of the shell was wound with wire coils, so as to harvest energy using PE-EM-TE hybrid effects from three-dimensional vibration, rotation and random human motion. The device was demonstrated to detect acceleration along the $\mathrm{x}, \mathrm{y}$ and $\mathrm{z}$ axes and angular velocity along the roll, pitch and yaw axes, which showed potential application towards human motion recognition for healthcare monitoring. 
To efficiently harvest wind energy, an ultra-compact and miniaturized windmill generator is illustrated in [168] based on the hybrid effects of TENG, PEH, and EMEH. At a wind speed of $6 \mathrm{~m} / \mathrm{s}$, the hybridized flexible blades of TENG and PEH generated maximal power of 1.67 and $1.38 \mathrm{~mW}$ at optimal loads of $10 \mathrm{M} \Omega$ and $330 \mathrm{~K} \Omega$, respectively. Simultaneously, the multipole-magnets of EMEH obtained a maximal power of $269 \mathrm{~mW}$ at $180 \Omega$. The authors demonstrated the feasibility of self-powered wireless sensor nodes from train movementinduced wind flow.

\section{E. Other hybrid energy harvesting systems}

Energy sources such as light, heat and vibrations co-exist in most conditions, and some of them may have limited accessibility and availability in certain cases. Take human beings as an example. Motion will be abundant when people are exercising or travelling but will be limited when at rest. Other energy sources, e.g. thermal or light energy, would provide an alternative. Therefore, in order to implement a resilient power supply, researchers have been investigating hybrid energy harvesting solutions that adopt multiple energy sources in one device [169-172]. Gambier et al. developed a hybrid harvester with layers of flexible solar pannel, piezoceramic, thin-film battery layers and a thermoelectric generator to harness multiple energy sources [173]. The flexible composite cantilever has approximate dimensions of $93 \times 25 \times 1.5 \mathrm{~mm}$ while the TEH is $30.5 \times 33 \times 4.1 \mathrm{~mm}$. A thin-film battery $(1 \mathrm{~mA} \cdot \mathrm{h})$ was able to be fully charged in 20 min using for a $223 \mathrm{~W} / \mathrm{m}^{2}$ solar irradiance, in 40 min for a $31{ }^{\circ} \mathrm{C}$ temperature difference and in $8 \mathrm{~h}$ for a $0.5 \mathrm{~g}$ and $56.4 \mathrm{~Hz}$ acceleration base input. Tan and Panda presented a hybrid harvester from indoor light and thermal energy with a shared power management circuit [169]. An average output power of $620 \mu \mathrm{W}$ was harvested at 1010 lux indoor solar irradiance of and a 10 $\mathrm{K}$ temperature gradient, which tripled the power that can be obtained by the thermal harvester. Yan and Yin developed a hybrid solar system that combines photovoltaic and thermoelectric mechanisms in a single device, as shown in Fig.15(a) [170]. Ambrosio et al. studied the combination of piezoelectric transducers, solar cells and a radio frequency (RF) antenna for 
multiple energy source harvesting, with a conclusion that the RF power at $2.4 \mathrm{GHz}$ is very limited compared to solar and vibration sources [124]. Chen et al. presented a lightweight, flexible and foldable hybrid energy harvesting solution for wearable applications by harnessing both light and kinetic energy [174]. Photovoltaic textile fabricated from lightweight polymer fibers and fiber based TE generators are combined to create a smart fabric with a thickness of $320 \mu \mathrm{m}$. This hybrid energy harvesting textile $(4 \times 5 \mathrm{~cm})$ had an average output power of 67 $\mu \mathrm{W}$ under sunlight in the presence of human motion and wind blowing. An example of such wearable smart textile is shown in Fig. 15(b) [175]. Bito et al. designed a hybrid solar and EM energy harvesting powered communication system (2.4 GHz ISM band) with a dual-port antenna, a solar cell, a power management unit and a controlling unit, as shown in Fig.15(e) [176]. The cold-start capability and low-power wireless sensing capability were experimentally validated. Yang et al. developed a hybrid harvester for self-powered condition monitoring of power grids, in which energy sources, including magnetic, thermoelectric and vibration, were adopted to provide a robust power supply [177]. A power management circuit for three energy sources was developed to convert the input energy into a DC output to energize a wireless sensor node.

Akan et al. explored hybrid energy harvesting technology on the system level for selfpowered IoT devices, where they illustrated the physical model of a representative IoT application in the smart grid infrastructure, enabled by a hybrid energy harvester [178]. The harvester prolonged the lifetime of the IoT network using multiple energy sources in the vicinity of the device. Wang et al. integrated light energy harvesting with wireless power transfer for hybrid wireless sensor networks [179]. A three-level network consisting of solar-powered, wireless-powered and mobile charger-powered nodes was studied to identify the number of sensors and locations for different energy sources and to obtain energy balance with the whole system.

Using one type of material to harvest multiple energy sources is another direction in hybrid energy harvesting. This is enabled by some materials that can provide both piezoelectric and 
pyroelectric effects [180]. Piezoelectric materials can generate electrical energy from mechanical strain, whereas pyroelectric materials generate electricity from temperature fluctuations. Since all pyroelectrics are piezoelectric, hybrid harvesters combining both piezoelectric and pyroelectric effects have been attempted to harness both temperature variation and applied stress. Kang and Yeatman presented a hybrid energy harvester using PZT-5H materials coated on a bi-metal beam, as shown in Fig.15(c) [105, 181]. A temperature oscillation was generated by an oscillating heat source to mimic the temperature changes in typical electronic chips. A bi-metal strip was adopted to generate mechanical deformation due to the variation of temperature to activate the piezoelectric effect. The human body is a location where motion and thermal gradients co-exist. Lee et al. developed a stretchable and flexible piezoelectric-pyroelectric harvester using micro-patterned piezoelectric polymer films, as shown in Fig.15(d) [182]. An enhanced performance was obtained by synchronizing the piezoelectric and pyroelectric dynamics by stretching-compressing and heating-cooling the materials in a controller manner, which means the frequency or phase of mechanical and thermal excitations have to be the same. As discussed by Sebald et al., in such a hybrid energy harvester design, the frequencies of temperature variation and mechanical vibration might be different, where the output voltages from both effects can be canceled in some conditions due to phase mismatching [183]. Appropriate power management circuit design and optimization are necessary to deal with this conflict. Mechanical vibration and changing magnetic field can be harnessed using a mass-spring-damper system with a permanent magnet as the proof mass $[184,185]$. Xu et al. presented a hybrid harvester using a piezoelectric cantilever beam with a magnetostrictive substrate to simultaneously collect the energy from mechanical motions and the magnetic field [184].

Designing power management circuits is of great importance to regulate electricity from different energy sources effectively into a form for sensing purposes. Katic et al. presented a dual-source power management interface that collects energy from a TEG and a implanted glucose biofuel cell [127]. The device performed a conversion efficiency of $89.5 \%$ (peak) at 66 
$\mu \mathrm{W}$ and the efficiency is over $80 \%$ if the power input is more than $9 \mu \mathrm{W}$. The interface can operate normally while the input voltages are over $10 \mathrm{mV}$ for $\mathrm{TEH}$ and $30 \mathrm{mV}$ for the glucose biofuel cell. The high conversion efficiency and low input voltage requirement allow such an interface to be practical in implantable applications. Bandyopadhyay and Chandrakasan designed a power management interface for energy sources including solar, thermal and vibration [128]. A dual-path structure was used, enhancing the peak efficiency of 11-13\% over the conventional two-stage method. A reconfigurable switch matrix that combines energy from photovoltaic, thermoelectric and piezoelectric conversions is implemented with the capability of handling a wide range of input voltage $(20 \mathrm{mV}-5 \mathrm{~V})$. Lhermet et al. developed a micropower architecture for harvesting thermal and RF energy [186]. An efficient power management and storage interface was realized with a $27 \mu \mathrm{A}$ charge current and a $12 \mathrm{pA}$ standby current. Bito et al. presented a $\mathrm{RF} /$ photovoltaic harvesting unit with an ultra-low input power requirement of $-25 \mathrm{dBm}(3 \mu \mathrm{W})[187]$.

In summary, a more resilient power supply can be achieved by harnessing different energy sources within one device. This has been explored by researchers for different energy sources in different applications. The challenges in hybrid energy harvesters include effective synergy of different conversion mechanisms to enhance the system performance, while maintaining the design compactness, and development of power management circuits that can handle different types of generated power with high efficiency. Advances in materials, system design and lowpower electronics would be also beneficial to the development of hybrid energy harvesting systems.

\section{Performance Comparison and Discussions}

In the previous section, we have introduced many specific examples of hybrid energy harvesting approaches. By adopting different structural configurations and materials from different energy sources, the hybrid generators exhibited various output characteristics. As the current research on hybrid systems is still in its infancy, it is hard to make quantitative analyses 
or comparative evaluations between different solutions. However, we can still find their common ground, make some qualitative comparisons, and provide inspiration for future investigation. In this section, the characteristics of the reported hybrid systems in configuration design and material selection will be summarized and explored, as well as the output performance under different excitation conditions.

PE-EM hybrid harvesters are mostly designed for vibration energy harvesting, but there are cases where this type is also used in harvesting human motion [116, 117], airflow [120, 122] and acoustic [121] energy sources, as summarized in Table I. The typical structure is a cantilever beam coated with piezoelectric material with a tip magnet [188]. A coil is placed near the tip magnet, and the configuration between the coil and tip magnet is either in the approachingseparation mode (ASM) [109, 119] or horizontal-sliding mode (HSM) [118] depending on the range of motion for the tip magnet. The HSM configuration is preferred in conditions where a large motion range is provided by the moving magnet. In order to enhance the bandwidth, nonlinear dynamics or multi-mode vibration are adopted. In addition to the cantilever structure, free-sliding magnets in a tube or guided rail are another common design for low-frequency motion energy harvesting. The sliding magnet introduces magnetic flux changes in coils and excites piezoelectric elements either by magnet forces [117] or direct impacts [116]. By integrating an airfoil with the cantilever structure, harvesters have also been developed to collect airflow energy [120, 122]. For PE-EM harvesters, the other critical challenge is to design effective power management circuits. As the publications in the literature are few, this comparison will be provided later, along with other types of hybrid harvesters. This also indicates that more efforts are needed in developing appropriate power management solutions for hybrid systems.

Table II shows a comparison of the reported PE-TE hybrid systems. From the perspective of energy sources, there are a lot of studies focused on the integration of piezoelectric and triboelectric materials with separated or shared electrodes. These simply apply an external force 
on the deformable laminated structures, such as arc-shape, r-shape or parallel plate, to achieve the deformation of the piezoelectric material, i.e., PVDF, and the contact-separation of the triboelectric materials, i.e., $\mathrm{Al}, \mathrm{Au}, \mathrm{Cu}$, and PDMS, PTFE [129-133]. Normally the hybrid maximum power density is less than tens of $\mathrm{mW} / \mathrm{cm}^{2}$ by an external force of less than ten Newtons with frequency less than $5 \mathrm{~Hz}$. The output power of the piezoelectric part is generally higher than the triboelectric part with the same dimension area, while the higher output voltage is mainly contributed by the triboelectric part. There are also some studies that achieved the PE-TE dual effect through the synthesis of composite materials, such as PDMS mixed with piezoelectric nano-particles or nano-fibers [134-138]. These works further expand the hybrid concept and provide new solutions for the hybrid systems. Nevertheless, the output performances of these hybrid PE-TE materials still need to be improved. Considering the utilization of environmental energy sources, application of external direct forces is generally not a suitable energy scenario. Therefore, researchers have developed spring-mass-stopper structures to collecting vibration energy, windmill blades to harness rotation energy, and flapping blades to harvest wind energy, which are more common in nature [139, 141-143]. Both the piezoelectric and triboelectric mechanisms can be realized with shared structural components. The authors have adopted these smart structures to achieve not only good mechanical characteristics, such as wide bandwidth, but also high electrical performance. It could be found that the output power of a hybrid PE-TE system is generally improved compared to a single piezoelectric or triboelectric device separately.

According to the characteristics of different vibration sources, various EM-TE hybrid harvesters have been developed to take advantage of the electromagnetic and triboelectric effects simultaneously in one single device. The working modes and output performances of the reported EM-TE hybrid systems are summarized in Table III. For instance, resonant-based spring-mass configurations are commonly used to harness vibrations with frequencies larger than $10 \mathrm{~Hz}$ [146-148]. To harvest ultra-low frequency vibrations of less than $5 \mathrm{~Hz}$, such as human motion or water waves, nonlinear or non-resonant moving-mass configurations are 
frequently implemented $[152,155-157,159]$. The common feature of these designs is that they use a magnetic mass or slider with surrounding fixed coils in approaching-separation or horizontal-sliding modes to generate the electromagnetic effect. The triboelectric effect is also induced during the mass movement in contact-separation, lateral-sliding or freestanding triboelectric-layer mode. To harvest rotation or wind flow energy, a rotating disk [144] or sleeve $[161,162]$ is used as a rotor to generate the relative motion, with the magnets and coils separately positioned at the rotor and the stator in HSM, while the triboelectric materials are usually arranged in LSM or FTM. For the above EM-TE hybrid systems, they share common moving mass or rotor components to simultaneously induce both electromagnetic and triboelectric effects. According to the output power and estimated dimensions from the references, the output power densities are calculated and normalized. It is found that the power densities of the EMEHs and TENGs are normally at the several or tens of $\mu \mathrm{W} / \mathrm{cm}^{3}$ level. Generally speaking, the power output of the EMEH part is usually higher than that of the TENG part, but at the cost of larger volume. Due to different electromagnetic and triboelectric integration schemes and structural configurations, as well as energy source conditions, it is hard to define which part contributes more to the same device. By the superimposition of the electromagnetic and triboelectric effects, the overall power output performance is mostly improved.

Table IV lists the triple-hybrid energy harvesting systems. The structural configurations are usually the superposition of the previous dual-hybrid configurations with piezoelectric, electromagnetic, and triboelectric effects. Under these circumstances, the output power could be mainly contributed by one or two energy conversion effects. It indicates that some energy conversion effect may not contribute enough energy to the hybrid device, but it may be conductive to improving the charging efficiency of a capacitor or acting as a self-powered sensing unit. It is still challenging to contribute considerable energy by three types of energy conversion effects in one hybrid energy harvesting system. 
Integrating different conversion mechanisms, such as photovoltaic, thermoelectric and piezoelectric, is also well presented in the literature to harness different energy sources, as summarized in Table V. Although more reliable power sources can be provided in hybrid systems, most studies put together different functional materials with limited synergy. Harvesting different energy sources using one conversion mechanism is one of the special ideas presented in the literature, such as using PZT ceramics to harness both vibration and thermal energies by piezoelectric and pyroelectric effects [105] or harnessing vibration and magnetic energy using a piezoelectric beam with a magnetostrictive mass [184].

Power management circuits are essential but challenging tasks for hybrid energy harvesting. Compared to the mechanical design and material synthesis, research on designing appropriate circuits for different hybrid systems is under-developed. Table VI summarizes the performance of the power management circuits presented in the literature for hybrid energy harvesters. This performance varies significantly for different energy sources and input conditions. It is indicated that no universal solutions are available now for different hybrid energy harvesters, but some common architectures or mechanisms have been demonstrated, such as inductor/converter sharing [126, 189], maximum power point tracking [169, 171], or synchronous control $[128,186]$. It is worth noting that the conversion efficiency in Table VI is just the conversion efficiency of the power management circuits rather than the efficiency for the whole energy harvesting system. The overall system-level efficiency will generally be substantially lower. The component- or system-level conversion efficiency and fundamental methods have been discussed in [190, 191]. In summary, conversion efficiency enhancement, electronic component count reduction and power loss reduction can all be realized in a power management circuit by designing the system in a holistic manner.

\section{Application Prospects for Hybrid Energy Harvesting Systems}

This section presents some tentative application areas for hybrid energy harvesters. The utilization of renewable hybrid energy sources, in the forms of light, heat, wind, wave, 
vibration, human motion, bioenergy, RF, radiation, etc., provides promising and long-term solutions for realizing self-sustained electronics, self-powered smart WSN systems and IoT devices in diverse applications. Application prospects for hybrid energy harvesting systems, will be generally discussed, including infrastructure health monitoring, industrial condition monitoring, smart transportation, human healthcare monitoring, marine monitoring, and aerospace engineering, as shown in Fig. 16.

\section{A. Infrastructure health monitoring}

There is consensus on the importance of monitoring and managing the health of civil infrastructure systems, e.g. water conservancy, power and communication facilities, roadway, railway, bridge, tunnel, buildings, agricultural facilities and environmental monitoring systems [192]. The arrangement of a large number of wireless sensor nodes for infrastructure health monitoring, which enables continuous detection or event-driven emergency response to longterm fatigue and instability, accidents, operational incidents, and natural hazards, which may ultimately save lives and reduce downtime and economy losses.

Since the infrastructure systems are normally in an outdoor environment, there are abundant renewable energy sources available besides traditional batteries or wired power supplies, for example, solar, wind flow, rain drop and RF energy. Up to now, we have seen the hybridization of energy harvesting systems from single or multiple energy sources with hybrid energy conversion mechanisms [193-196]. Qian and Jing [161] reported a wind-driven hybridized EM-TE harvester and a solar cell for self-powered condition monitoring of natural disasters. A temperature sensor and a vibration sensor were adopted for fire detection and earthquake monitoring, respectively. Liu et al. [195] developed a thermoelectric and electromagnetic hybrid energy harvester from fluid motion and temperature variation in an irrigating systems. In an intelligent agriculture irrigation system, various sensor nodes or monitors, e.g., temperature sensors and flow meters, can be integrated with the power supply from renewable energy harvesters. Further efforts are still needed to support the long-term self- 
powered WSN applications for smart buildings, intelligent agriculture, structural health monitoring, security and facility monitoring, environmental monitoring, and so on.

\section{B. Industry condition monitoring}

It is generally known that condition monitoring is necessary for modern production and manufacturing processes, especially for smart factories in Industry 4.0 (the fourth technological revolution) in the future. Since industry cannot afford any unexpected downtime caused by machine malfunction, condition monitoring systems feedback a series of parameters of the machinery (vibration, temperature, voltage, current, etc.) to allow early detection and identification of machine and system irregularities in real time. More than this, these machine health insights result in improved efficiency, accelerating the path to Industry 4.0. WSNs have the advantages of high flexibility and low power consumption in realizing real-time remote condition monitoring. Hybrid energy harvesting technologies collect the unused energy from machines or the ambient environments to power the sensor nodes, avoiding the expensive cost and difficult task of recharging or replacing batteries especially at the remote or inaccessible conditions [197]. He et al. [166] reported a hybridized PE-EM-TE vibrational energy harvesting device with high power output. The device was integrated into a wireless sensor of temperature and vibration, so as to monitor the bearing condition to avoid mechanical fatigue or overload. It is of great importance to the application of the industry IoTs in the future. Yang et al. [177] presented a magnetic, piezoelectric and thermoelectric hybrid energy harvester from the industry power equipment, which provides a steady DC output voltage that was fed into a ZigBee sensor to keep it operational without downtime.

\section{Smart transportation}

Electrification and autonomous driving are the current trends for transportation, especially for the automotive industry. Sensors and electronics will be embedded within structures on a much larger scale to satisfy the digitalization and automation demands. Using different energy sources can provide a more robust and powerful power supply solution. Transportation vehicles, such as cars, trains, buses, ferries, and airplanes, are subject to a variety of available 
energy sources [198, 199]. Hybrid energy harvesting systems could provide self-powered sensing solutions for condition monitoring and event detection in transportation vehicles [200, 201]. Energy harvesters using single energy sources or single conversion mechanisms have been widely studied in this area [156, 202], but hybrid systems are not much reported, with few examples. In transportation systems, vibration and airflow are abundant at a relatively high energy levels and frequency range, and the size constraint is often less demanding compared to some applications such as biomedical devices. Conversion mechanisms including PE and EM are suitable. TE can also be used in conditions such as tire pressure monitoring, but the material reliability is one of the concerns. Other energy sources such as thermal and solar can also be used, but they have more strict requirements on the locations where the harvesters can be mounted.

\section{Human healthcare monitoring}

The development of wearable and implantable electronic devices can assist healthcare professionals to intervene chronic diseases as early as possible and facilitate real-time remote monitoring of patients' vital signs. One of the main bottlenecks is the sustainable power supply. In fact, there could be many energy sources available in or on a human body, including human motion, muscle contraction, body heat, blood pulsation, cardiac/lung motions, etc. In order to scavenging these human body energy, a variety of energy harvesting configurations have been presented for recharging batteries and eventually realizing of self-powered wearable or implantable electronic devices [203-206]. As an example, by using hybrid PE-TE effects from human walking, Zhu et al. [207] have presented self-functional socks to realize self-powered sensing of various physiological signals, such as contact force, gait, sweat level and so on.

In terms of conversion mechanisms, all the above-mentioned mechanisms have their advantages in different areas or sources from human body. For example, the mechanical energy available from footsteps large, so this could be ideal for EM and TE [208-210], whereas PV and TEG would be good options for upper body and skin-related applications [174, 211, 212]. However, compared to other applications, energy sources from the human body normally exist 
in a low-grade or low-frequency and random form. It is more challenging to design effective solutions to generate sufficient energy for sensing in a limited space. Hybrid systems either using multiple conversion mechanisms or multiple energy sources might provide solutions to tackle this challenge.

\section{E. Marine monitoring and development}

Marine monitoring and development have received increasing attention from various countries and research organizations. The operating life of marine equipment is largely limited by the power supply, more specifically depending on the capacity of the carried battery. It has long been noted that there is a large amount of renewable energy in the ocean, such as solar energy, wind energy, tidal energy, water wave energy, water flow energy, temperature gradient energy, and salinity gradient energy, which has been estimated to total over 75 TW around the world $[213,214]$. In past few years, a number of researchers have been attracted to study the so-called blue energy harvesting techniques based on various transduction mechanisms, which are considered and demonstrated as potential supplies for self-powered sensors or self-charging batteries in marine monitoring or sea development systems $[215,216]$. Some researchers proposed EM-TE hybridized water wave harvesters based on a magnetic sphere [217], or chaotic pendulum [218], to power the sensor nodes of the ocean monitoring buoys. Wang et al. [156] not only rendered a ship-shaped EM-TE hybrid device on water-wave energy harvesting, but also presented an example towards self-desalination and self-powered marine rescue system. Wang et al. [219] described a blue energy dream that the integration of water wave power generators, wind turbines and solar panels can form a power generation network above the water surface. We believe the hybrid blue energy harvesting systems could have great potential in the self-powered IoT for marine monitoring and development.

\section{F. Aerospace engineering}

Monitoring the structural integrity and operating conditions of aircraft and spacecraft in real-time is critical to enhance passenger safety and to reduce operational downtime and maintenance costs $[220,221]$. Energy harvesting could provide reliable energy sources for 
those distributed wireless sensors monitoring the operational conditions. In aircraft and spacecraft, there are also numerous energy sources available, especially solar, vibration and temperature difference. Le et al. provide a comprehensive review on available energy sources and technologies for energy harvesting in aeronautical applications, especially on vibrational and thermal sources [222]. The propulsion system provides the primary source of internal vibration. The frequency and amplitude range vary significantly depending on the rotor speed and blade passage frequency in both helicopters and propeller-driven aircraft. The vibration from typical jet engines has a frequency range from $20 \mathrm{~Hz}$ to $500 \mathrm{~Hz}$ with a peak acceleration of $3 \mathrm{~g}$ [223]. In the case of thermal sources, engines, hydraulics, and gear trains are all heat sources. The temperature difference between cabin and fuselage is also a possibility to energize sensors mounted on the fuselage. Kiziroglou et al. used heat mass to create a temperature difference for a TEG using the temperature different in the fuselage before and after take-off [87]. Recently, Wang et al. developed a self-powered monitoring system for jet engines using a nonlinear piezoelectric harvester [224]. A 22-gram energy harvester could produce $79 \mathrm{~mW}$ output power at $2050 \mathrm{rpm}$ rotation conditions with a $100 \mathrm{k} \Omega$ load. In astronautical engineering, solar, electromagnetic field and diurnal temperature variation in space can be potential energy sources in addition to the energy sources on spacecraft. Energy harvesting in this area is generally limited due to the special requirements in such environments, but hybrid energy harvesting could be a potential enabling technology for autonomous and distributed sensing in astronautical engineering.

\section{Concluding Remarks}

In view of the above hybrid energy harvesting systems, we have seen many interesting and exciting demonstrations. Researchers have designed and employed various materials, configurations and mechanisms according to the characteristics of different energy sources and application scenarios. Through adopting two or more energy conversion effects or energy sources, hybrid energy harvesters have achieved a certain degree of improvement in energy 
output. In this review, a broad range of energy sources and conversion mechanisms are discussed and reviewed, with the vibrational and/or thermal hybrid energy harvesters being the main review focus. Vibrational and thermal hybrid harvesters also represent the mainstream in hybrid energy harvesting, which also indicates that more efforts are required to explore different possibilities in adopting different conversion mechanisms and energy sources. Since hybrid energy harvesting technology is still in its infancy, there is relatively little comparison and discussion on the hybrid energy harvesting mechanisms compared to single ones in the current literature. In this review, we have summarized the output performance of the reported typical hybrid systems; qualitative remarks can be found in the tables. Because the input and output characteristics given by the authors do not have a unified specification standard, and the system is greatly affected by various input quantities, it is not yet possible to judge which structure or mechanism is better. How much the power density and energy conversion efficiency can be improved via hybrid energy harvesting technology remains to be further studied. This is indeed a very challenging task, since there are so many influencing factors and working conditions, and it is necessary to find some normalized measurement indices or key criteria. Researchers should be aware of the risk of developing devices simply for the sake of showing hybrid effects, but at a cost of additional structural complexity and increased device volume which is not justified by the enhanced performance. We believe that investigation of hybrid energy harvesting technologies will remain a hot topic and the next decade will witness rapid growth of hybrid energy harvesting systems in a variety of applications. 


\section{Acknowledgement}

The authors acknowledge financial support from the following research grants: National Key Research and Development Program of China (Grant No. 2019YFB2004800), National Natural Science Foundation of China (Grant No. 51875377). 


\section{Reference}

[1] Guo S, Liu Q, Sun J, Jin H. A review on the utilization of hybrid renewable energy. Renewable and Sustainable Energy Reviews. 2018;91:1121-47.

[2] Nematollahi O, Hoghooghi H, Rasti M, Sedaghat A. Energy demands and renewable energy resources in the Middle East. Renewable and Sustainable Energy Reviews. 2016;54:1172-81.

[3] Niblick B, Landis AE. Assessing renewable energy potential on United States marginal and contaminated sites. Renewable and Sustainable Energy Reviews. 2016;60:489-97.

[4] Panwar NL, Kaushik SC, Kothari S. Role of renewable energy sources in environmental protection: A review. Renewable and Sustainable Energy Reviews. 2011;15:1513-24.

[5] Scarlat N, Dallemand J-F, Monforti-Ferrario F, Banja M, Motola V. Renewable energy policy framework and bioenergy contribution in the European Union - An overview from National Renewable Energy Action Plans and Progress Reports. Renewable and Sustainable Energy Reviews. 2015;51:96985.

[6] Dewan A, Ay SU, Karim MN, Beyenal H. Alternative power sources for remote sensors: A review. Journal of Power Sources. 2014;245:129-43.

[7] Misra V, Bozkurt A, Calhoun B, Jackson T, Jur J, Lach J, et al. Flexible Technologies for Self-Powered Wearable Health and Environmental Sensing. Proceedings of the IEEE. 2015;103:665-81.

[8] Mitcheson PD, Yeatman EM, Rao GK, Holmes AS, Green TC. Energy Harvesting From Human and Machine Motion for Wireless Electronic Devices. Proceedings of the IEEE. 2008;96:1457-86.

[9] Shaikh FK, Zeadally S. Energy harvesting in wireless sensor networks: A comprehensive review. Renewable and Sustainable Energy Reviews. 2016;55:1041-54.

[10] Beeby SP, Tudor MJ, White NM. Energy harvesting vibration sources for microsystems applications. Measurement Science and Technology. 2006;17:R175-R95.

[11] Selvan KV, Mohamed Ali MS. Micro-scale energy harvesting devices: Review of methodological performances in the last decade. Renewable and Sustainable Energy Reviews. 2016;54:1035-47.

[12] Vullers RJM, van Schaijk R, Doms I, Van Hoof C, Mertens R. Micropower energy harvesting. SolidState Electronics. 2009;53:684-93.

[13] Wei C, Jing X. A comprehensive review on vibration energy harvesting: Modelling and realization. Renewable and Sustainable Energy Reviews. 2017;74:1-18.

[14] Bai Y, Jantunen H, Juuti J. Energy Harvesting Research: The Road from Single Source to Multisource. Adv Mater. 2018:e1707271.

[15] Ryu H, Yoon HJ, Kim SW. Hybrid Energy Harvesters: Toward Sustainable Energy Harvesting. Adv Mater. 2019:e1802898.

[16] Bowen CR, Kim HA, Weaver PM, Dunn S. Piezoelectric and ferroelectric materials and structures for energy harvesting applications. Energy \& Environmental Science. 2014;7:25-44.

[17] Liu H, Zhong J, Lee C, Lee S-W, Lin L. A comprehensive review on piezoelectric energy harvesting technology: Materials, mechanisms, and applications. Applied Physics Reviews. 2018;5:041306.

[18] Toprak A, Tigli O. Piezoelectric energy harvesting: State-of-the-art and challenges. Applied Physics Reviews. 2014;1:031104.

[19] Yang Z, Zhou S, Zu J, Inman D. High-Performance Piezoelectric Energy Harvesters and Their Applications. Joule. 2018;2:642-97.

[20] Arnold DP. Review of Microscale Magnetic Power Generation. IEEE Transactions on Magnetics. 2007;43:3940-51.

[21] Wang ZL, Chen J, Lin L. Progress in triboelectric nanogenerators as a new energy technology and self-powered sensors. Energy \& Environmental Science. 2015;8:2250-82.

[22] Kishore RA, Priya S. A Review on Low-Grade Thermal Energy Harvesting: Materials, Methods and Devices. Materials. 2018;11.

[23] Thakre A, Kumar A, Song H-C, Jeong D-Y, Ryu J. Pyroelectric energy conversion and its applications-flexible energy harvesters and sensors. Sensors. 2019;19:2170.

[24] Siddique ARM, Mahmud S, Heyst BV. A comprehensive review on vibration based micro power generators using electromagnetic and piezoelectric transducer mechanisms. Energy Conversion and Management. 2015;106:728-47.

[25] Zheng Q, Shi B, Li Z, Wang ZL. Recent Progress on Piezoelectric and Triboelectric Energy Harvesters in Biomedical Systems. Advanced Science. 2017;4:1700029. 
[26] Lee B-Y, Kim DH, Park J, Park K-I, Lee KJ, Jeong CK. Modulation of surface physics and chemistry in triboelectric energy harvesting technologies. Science and technology of advanced materials. 2019;20:758-73.

[27] Hong C-H, Kim H-P, Choi B-Y, Han H-S, Son JS, Ahn CW, et al. Lead-free piezoceramics-Where to move on? Journal of Materiomics. 2016;2:1-24.

[28] Daqaq MF, Masana R, Erturk A, Dane Quinn D. On the role of nonlinearities in vibratory energy harvesting: a critical review and discussion. Applied Mechanics Reviews. 2014;66.

[29] Hou Y, Zhou Y, Yang L, Li Q, Zhang Y, Zhu L, et al. Flexible ionic diodes for low - frequency mechanical energy harvesting. Advanced Energy Materials. 2017;7:1601983.

[30] Deng Q, Kammoun M, Erturk A, Sharma P. Nanoscale flexoelectric energy harvesting. International Journal of Solids and Structures. 2014;51:3218-25.

[31] Li W, Torres D, Díaz R, Wang Z, Wu C, Wang C, et al. Nanogenerator-based dual-functional and selfpowered thin patch loudspeaker or microphone for flexible electronics. Nature communications. 2017;8:15310.

[32] Kim S, Choi SJ, Zhao K, Yang H, Gobbi G, Zhang S, et al. Electrochemically driven mechanical energy harvesting. Nature communications. 2016;7:1-7.

[33] Tang Y, Zheng Q, Chen B, Ma Z, Gong S. A new class of flexible nanogenerators consisting of porous aerogel films driven by mechanoradicals. Nano energy. 2017;38:401-11.

[34] Chae I, Jeong CK, Ounaies Z, Kim SH. Review on electromechanical coupling properties of biomaterials. ACS Applied Bio Materials. 2018;1:936-53.

[35] Bai $Y$, Jantunen H, Juuti J. Hybrid, Multi-Source, and Integrated Energy Harvesters. Frontiers in Materials. 2018;5.

[36] Chandrasekaran S, Bowen C, Roscow J, Zhang Y, Dang DK, Kim EJ, et al. Micro-scale to nano-scale generators for energy harvesting: Self powered piezoelectric, triboelectric and hybrid devices. Physics Reports. 2019;792:1-33.

[37] Lee J-H, Kim J, Kim TY, Al Hossain MS, Kim S-W, Kim JH. All-in-one energy harvesting and storage devices. Journal of Materials Chemistry A. 2016;4:7983-99.

[38] Khan AA, Mahmud A, Ban D. Evolution From Single to Hybrid Nanogenerator: A Contemporary Review on Multimode Energy Harvesting for Self-Powered Electronics. IEEE Transactions on Nanotechnology. 2019;18:21-36.

[39] Toprak A, Tigli O. Piezoelectric energy harvesting: State-of-the-art and challenges. Applied Physics Reviews. 2014;1.

[40] Roundy S, Wright PK. A piezoelectric vibration based generator for wireless electronics. Smart Materials and Structures. 2004;13:1131-42.

[41] Huang M, Hou C, Li Y, Liu H, Wang F, Chen T, et al. A Low-Frequency MEMS Piezoelectric Energy Harvesting System Based on Frequency Up-Conversion Mechanism. Micromachines (Basel). 2019;10.

[42] Fu H, Yeatman EM. A methodology for low-speed broadband rotational energy harvesting using piezoelectric transduction and frequency up-conversion. Energy. 2017;125:152-61.

[43] Zhang X, Wang P, Liu X, Zhang W, Zhong Y, Zhao H, et al. Effect of post-annealing on microstructure and piezoelectric properties of $\mathrm{ZnO}$ thin film for triangular shaped vibration energy harvester. Surface and Coatings Technology. 2019;361:123-9.

[44] Xie XD, Carpinteri A, Wang Q. A theoretical model for a piezoelectric energy harvester with a tapered shape. Engineering Structures. 2017;144:19-25.

[45] Liu H, Lee C, Kobayashi T, Tay CJ, Quan C. A new S-shaped MEMS PZT cantilever for energy harvesting from low frequency vibrations below $30 \mathrm{~Hz}$. Microsystem Technologies. 2012;18:497-506.

[46] Yang Z, Wang YQ, Zuo L, Zu J. Introducing arc-shaped piezoelectric elements into energy harvesters. Energy Conversion and Management. 2017;148:260-6.

[47] Yesner G, Jasim A, Wang H, Basily B, Maher A, Safari A. Energy harvesting and evaluation of a novel piezoelectric bridge transducer. Sensors and Actuators A: Physical. 2019;285:348-54.

[48] Yang Z, Zu J. High-efficiency compressive-mode energy harvester enhanced by a multi-stage force amplification mechanism. Energy conversion and management. 2014;88:829-33.

[49] Cheng S, Arnold DP. A study of a multi-pole magnetic generator for low-frequency vibrational energy harvesting. Journal of Micromechanics and Microengineering. 2010;20.

[50] Wang P, Liu H, Dai X, Yang Z, Wang Z, Zhao X. Design, simulation, fabrication and characterization of a micro electromagnetic vibration energy harvester with sandwiched structure and air channel. Microelectronics Journal. 2012;43:154-9. 
[51] Liu H, Soon BW, Wang N, Tay CJ, Quan C, Lee C. Feasibility study of a 3D vibration-driven electromagnetic MEMS energy harvester with multiple vibration modes. Journal of Micromechanics and Microengineering. 2012;22:125020.

[52] Arnold DP, Das S, Park JW, Zana I, Lang JH, Allen MG. Microfabricated High-Speed Axial-Flux Multiwatt Permanent-Magnet Generators-Part II: Design, Fabrication, and Testing. Journal of Microelectromechanical Systems. 2006;15:1351-63.

[53] Beeby SP, Torah RN, Tudor MJ, Glynne-Jones P, O'Donnell T, Saha CR, et al. A micro electromagnetic generator for vibration energy harvesting. Journal of Micromechanics and Microengineering. 2007;17:1257-65.

[54] Yang B, Lee C, Xiang W, Xie J, Han He J, Kotlanka RK, et al. Electromagnetic energy harvesting from vibrations of multiple frequencies. Journal of Micromechanics and Microengineering. 2009;19:035001. [55] Zhang Y, Cao J, Zhu H, Lei Y. Design, modeling and experimental verification of circular Halbach electromagnetic energy harvesting from bearing motion. Energy Conversion and Management. 2019;180:811-21.

[56] Li Z, Yan Z, Luo J, Yang Z. Performance comparison of electromagnetic energy harvesters based on magnet arrays of alternating polarity and configuration. Energy Conversion and Management. 2019;179:132-40.

[57] Li Y, Guo Q, Liu H, Chen T, Sun L, Ma X, et al. Design and Experiment of an Ultra-Low Frequency Pendulum-Based Wave Energy Harvester. 2019 IEEE 14th International Conference on Nano/Micro Engineered and Molecular Systems (NEMS)2019. p. 101-4.

[58] Liu H, Hou C, Lin J, Li Y, Shi Q, Chen T, et al. A non-resonant rotational electromagnetic energy harvester for low-frequency and irregular human motion. Applied Physics Letters. 2018;113:203901.

[59] YulongZhang, AnxinLuo, YifanWang, XiangtianDai, YanLu, FeiWang. Rotational electromagnetic energy harvester for human motion application at low frequency. Applied Physics Letters. 2020;116:053902.

[60] Fu H, Theodossiades S, Gunn B, Abdallah I, Chatzi E. Ultra-low frequency energy harvesting using bi-stability and rotary-translational motion in a magnet-tethered oscillator. Nonlinear Dynamics. 2020. [61] Wang ZL. Triboelectric Nanogenerators as New Energy Technology for Self-Powered Systems and as Active Mechanical and Chemical Sensors. ACS Nano. 2013;7:9533-57.

[62] Zhu G, Lin ZH, Jing Q, Bai P, Pan C, Yang Y, et al. Toward large-scale energy harvesting by a nanoparticle-enhanced triboelectric nanogenerator. Nano Lett. 2013;13:847-53.

[63] Wang S, Lin L, Wang ZL. Nanoscale triboelectric-effect-enabled energy conversion for sustainably powering portable electronics. Nano Lett. 2012;12:6339-46.

[64] Wang S, Lin L, Xie Y, Jing Q, Niu S, Wang ZL. Sliding-triboelectric nanogenerators based on in-plane charge-separation mechanism. Nano Lett. 2013;13:2226-33.

[65] Lin L, Wang S, Xie Y, Jing Q, Niu S, Hu Y, et al. Segmentally structured disk triboelectric nanogenerator for harvesting rotational mechanical energy. Nano letters. 2013;13:2916-23.

[66] Bai P, Zhu G, Liu Y, Chen J, Jing Q, Yang W, et al. Cylindrical Rotating Triboelectric Nanogenerator. ACS Nano. 2013;7:6361-6.

[67] Yang Y, Zhou YS, Zhang H, Liu Y, Lee S, Wang ZL. A single-electrode based triboelectric nanogenerator as self-powered tracking system. Adv Mater. 2013;25:6594-601.

[68] Li Y, Cheng G, Lin Z-H, Yang J, Lin L, Wang ZL. Single-electrode-based rotationary triboelectric nanogenerator and its applications as self-powered contact area and eccentric angle sensors. Nano Energy. 2015;11:323-32.

[69] Qiu C, Wu F, Lee C, Yuce MR. Self-powered control interface based on Gray code with hybrid triboelectric and photovoltaics energy harvesting for loT smart home and access control applications. Nano Energy. 2020;70:104456.

[70] Wang S, Niu S, Yang J, Lin L, Wang ZL. Quantitative Measurements of Vibration Amplitude Using a Contact-Mode Freestanding Triboelectric Nanogenerator. ACS Nano. 2014 8:12004-13

[71] Lin L, Wang S, Niu S, Liu C, Xie Y, Wang ZL. Noncontact free-rotating disk triboelectric nanogenerator as a sustainable energy harvester and self-powered mechanical sensor. ACS applied materials \& interfaces. 2014;6:3031-8.

[72] DiSalvo FJJS. Thermoelectric cooling and power generation. 1999;285:703-6.

[73] Bell LEJS. Cooling, heating, generating power, and recovering waste heat with thermoelectric systems. 2008;321:1457-61. 
[74] Sundarraj P, Maity D, Roy SS, Taylor RAJRA. Recent advances in thermoelectric materials and solar thermoelectric generators-a critical review. 2014;4:46860-74.

[75] Goldsmid HJ. Introduction to thermoelectricity: Springer; 2010.

[76] Hudak NS, Amatucci GGJJoAP. Small-scale energy harvesting through thermoelectric, vibration, and radiofrequency power conversion. 2008;103:5.

[77] Yang L, Chen Z-G, Dargusch MS, Zou J. High Performance Thermoelectric Materials: Progress and Their Applications. 2018;8:1701797.

[78] Chen Z-G, Shi X, Zhao L-D, Zou J. High-performance SnSe thermoelectric materials: Progress and future challenge. Progress in Materials Science. 2018;97:283-346.

[79] Yang J. Potential applications of thermoelectric waste heat recovery in the automotive industry. ICT 2005 24th International Conference on Thermoelectrics, 20052005. p. 170-4.

[80] Kishore RA, Nozariasbmarz A, Poudel B, Sanghadasa M, Priya S. Ultra-high performance wearable thermoelectric coolers with less materials. Nature Communications. 2019;10:1765.

[81] Leonov V. Thermoelectric Energy Harvesting of Human Body Heat for Wearable Sensors. IEEE Sensors Journal. 2013;13:2284-91.

[82] Xi H, Luo L, Fraisse G. Development and applications of solar-based thermoelectric technologies. Renewable and Sustainable Energy Reviews. 2007;11:923-36.

[83] Baranowski LL, Snyder GJ, Toberer ES. Concentrated solar thermoelectric generators. Energy \& Environmental Science. 2012;5:9055-67.

[84] Kraemer D, Poudel B, Feng H-P, Caylor JC, Yu B, Yan X, et al. High-performance flat-panel solar thermoelectric generators with high thermal concentration. Nature materials. 2011;10:532-8.

[85] Rowe DM. Applications of nuclear-powered thermoelectric generators in space. Applied Energy. 1991;40:241-71.

[86] Kim SJ, Lee HE, Choi H, Kim Y, We JH, Shin JS, et al. High-performance flexible thermoelectric power generator using laser multiscanning lift-off process. 2016;10:10851-7.

[87] Kiziroglou ME, Wright SW, Toh TT, Mitcheson PD, Becker T, Yeatman EMJITolE. Design and fabrication of heat storage thermoelectric harvesting devices. 2013;61:302-9.

[88] Samson D, Kluge M, Becker T, Schmid U. Wireless sensor node powered by aircraft specific thermoelectric energy harvesting. Sensors and Actuators A: Physical. 2011;172:240-4.

[89] Kiziroglou ME, Becker T, Wright SW, Yeatman EM, Evans JW, Wright PK. Three-Dimensional Printed Insulation For Dynamic Thermoelectric Harvesters With Encapsulated Phase Change Materials. IEEE Sensors Letters. 2017;1:1-4.

[90] Zhang Q, Agbossou A, Feng Z, Cosnier MJS, Physical AA. Solar micro-energy harvesting based on thermoelectric and latent heat effects. Part II: Experimental analysis. 2010;163:284-90.

[91] Verma G, Sharma V. A Novel Thermoelectric Energy Harvester for Wireless Sensor Network Application. IEEE Transactions on Industrial Electronics. 2019;66:3530-8.

[92] Trelles JP, Dufly JJ. Numerical simulation of porous latent heat thermal energy storage for thermoelectric cooling. Appl Therm Eng. 2003;23:1647-64.

[93] Lingam D, Parikh AR, Huang J, Jain A, Minary-Jolandan M. Nano/microscale pyroelectric energy harvesting: challenges and opportunities. International Journal of Smart and Nano Materials. 2013;4:229-45.

[94] Bowen C, Kim H, Weaver P, Dunn SJE, Science E. Piezoelectric and ferroelectric materials and structures for energy harvesting applications. 2014;7:25-44.

[95] Lang SBJPt. Pyroelectricity: from ancient curiosity to modern imaging tool. 2005;58:31.

[96] Cuadras A, Gasulla M, Ferrari V. Thermal energy harvesting through pyroelectricity. Sensors and Actuators A: Physical. 2010;158:132-9.

[97] Cha G, Jia Y, Ju YS. High-power density pyroelectric energy harvesters incorporating switchable liquid-based thermal interfaces. 2012 IEEE 25th International Conference on Micro Electro Mechanical Systems (MEMS): IEEE; 2012. p. 1241-4.

[98] Meuel T, Coudert M, Fischer P, Bruneau CH, Kellay H. Effects of rotation on temperature fluctuations in turbulent thermal convection on a hemisphere. Scientific Reports. 2018;8:16513.

[99] Xie M, Dunn S, Le Boulbar E, Bowen CR. Pyroelectric energy harvesting for water splitting. International Journal of Hydrogen Energy. 2017;42:23437-45.

[100] Zhang Y, Kumar S, Marken F, Krasny M, Roake E, Eslava S, et al. Pyro-electrolytic water splitting for hydrogen generation. Nano Energy. 2019;58:183-91. 
[101] Mane P, Xie J, Leang KK, Mossi KJItou, ferroelectrics,, control f. Cyclic energy harvesting from pyroelectric materials. 2011;58:10-7.

[102] Kim J, Yamanaka S, Murayama I, Katou T, Sakamoto T, Kawasaki T, et al. Pyroelectric power generation from the waste heat of automotive exhaust gas. 2020.

[103] Kim J, Yamanaka S, Murayama I, Katou T, Sakamoto T, Kawasaki T, et al. Pyroelectric power generation from the waste heat of automotive exhaust gas. Sustainable Energy \& Fuels. 2020;4:11439.

[104] Ali HM, Arshad A, Janjua MM, Baig W, Sajjad U. Thermal performance of LHSU for electronics under steady and transient operations modes. International Journal of Heat and Mass Transfer. 2018;127:1223-32.

[105] Kang M, Yeatman EM. Coupling of piezo- and pyro-electric effects in miniature thermal energy harvesters. Applied Energy. 2020;262:114496.

[106] Hunter SR, Lavrik NV, Bannuru T, Mostafa S, Rajic S, Datskos PG. Development of MEMS based pyroelectric thermal energy harvesters. Energy Harvesting and Storage: Materials, Devices, and Applications II: International Society for Optics and Photonics; 2011. p. 80350V.

[107] Zabek D, Taylor J, Ayel V, Bertin Y, Romestant C, Bowen CR. A novel pyroelectric generator utilising naturally driven temperature fluctuations from oscillating heat pipes for waste heat recovery and thermal energy harvesting. 2016;120:024505.

[108] Ravindran SKT, Huesgen T, Kroener M, Woias P. A self-sustaining micro thermomechanicpyroelectric generator. Applied Physics Letters. 2011;99:104102.

[109] Toyabur RM, Salauddin M, Cho H, Park JY. A multimodal hybrid energy harvester based on piezoelectric-electromagnetic mechanisms for low-frequency ambient vibrations. Energy Conversion and Management. 2018;168:454-66.

[110] Yang B, Lee C, Kee WL, Lim S-P. Hybrid energy harvester based on piezoelectric and electromagnetic mechanisms. J of Micro/Nanolithography, MEMS, and MOEMS. 2010;9:1-10, .

[111] Xia H, Chen R, Ren L. Analysis of piezoelectric-electromagnetic hybrid vibration energy harvester under different electrical boundary conditions. Sensors and Actuators A: Physical. 2015;234:87-98.

[112] Li P, Gao S, Niu S, Liu H, Cai H. An analysis of the coupling effect for a hybrid piezoelectric and electromagnetic energy harvester. Smart Materials and Structures. 2014;23:065016.

[113] Mahmoudi S, Kacem N, Bouhaddi N. Enhancement of the performance of a hybrid nonlinear vibration energy harvester based on piezoelectric and electromagnetic transductions. Smart Materials and Structures. 2014;23:075024.

[114] Xia H, Chen R, Ren L. Parameter tuning of piezoelectric-electromagnetic hybrid vibration energy harvester by magnetic force: Modeling and experiment. Sensors and Actuators A: Physical. 2017;257:73-83.

[115] Xu Z, Shan X, Chen D, Xie T. A Novel Tunable Multi-Frequency Hybrid Vibration Energy Harvester Using Piezoelectric and Electromagnetic Conversion Mechanisms. Applied Sciences. 2016;6:10.

[116] Hamid R, Yuce MR. A wearable energy harvester unit using piezoelectric-electromagnetic hybrid technique. Sensors and Actuators A: Physical. 2017;257:198-207.

[117] Fan K, Liu S, Liu H, Zhu Y, Wang W, Zhang D. Scavenging energy from ultra-low frequency mechanical excitations through a bi-directional hybrid energy harvester. Applied Energy. 2018;216:820.

[118] Li Z, Li T, Yang Z, Naguib HE. Toward a 0.33 W piezoelectric and electromagnetic hybrid energy harvester: Design, experimental studies and self-powered applications. Applied Energy. 2019;255:113805.

[119] Yang T, Cao Q. Dynamics and performance evaluation of a novel tristable hybrid energy harvester for ultra-low level vibration resources. International Journal of Mechanical Sciences. 2019;156:123-36. [120] Dias JAC, De Marqui C, Erturk A. Hybrid piezoelectric-inductive flow energy harvesting and dimensionless electroaeroelastic analysis for scaling. Applied Physics Letters. 2013;102:044101.

[121] Khan FU, Izhar. Hybrid acoustic energy harvesting using combined electromagnetic and piezoelectric conversion. Review of Scientific Instruments. 2016;87:025003.

[122] Iqbal M, Khan FU. Hybrid vibration and wind energy harvesting using combined piezoelectric and electromagnetic conversion for bridge health monitoring applications. Energy Conversion and Management. 2018;172:611-8.

[123] Zhao L-C, Zou H-X, Yan G, Liu F-R, Tan T, Zhang W-M, et al. A water-proof magnetically coupled piezoelectric-electromagnetic hybrid wind energy harvester. Applied Energy. 2019;239:735-46. 
[124] Ambrosio R, TorreAlba R, Guerrero-C JF, González V, Limon A, Moreno M. Energy harvesting combining three different sources for low power applications. 2015 12th International Conference on Electrical Engineering, Computing Science and Automatic Control (CCE)2015. p. 1-6.

[125] Umaz R, Sahin Y. An Architecture for Two Ambient Energy Sources. 2019 4th International Conference on Power Electronics and their Applications (ICPEA)2019. p. 1-6.

[126] Chamanian S, Çiftci B, Uluşan H, Muhtaroğlu A, Külah H. Power-Efficient Hybrid Energy Harvesting System for Harnessing Ambient Vibrations. IEEE Transactions on Circuits and Systems I: Regular Papers. 2019;66:2784-93.

[127] Katic J, Rodriguez S, Rusu A. A High-Efficiency Energy Harvesting Interface for Implanted Biofuel Cell and Thermal Harvesters. IEEE Transactions on Power Electronics. 2018;33:4125-34.

[128] Bandyopadhyay S, Chandrakasan AP. Platform Architecture for Solar, Thermal, and Vibration Energy Combining With MPPT and Single Inductor. IEEE Journal of Solid-State Circuits. 2012;47:2199215.

[129] Jung WS, Kang MG, Moon HG, Baek SH, Yoon SJ, Wang ZL, et al. High output piezo/triboelectric hybrid generator. Sci Rep. 2015;5:9309.

[130] Wang X, Yang B, Liu J, Yang C. A transparent and biocompatible single-friction-surface triboelectric and piezoelectric generator and body movement sensor. Journal of Materials Chemistry $A$. 2017;5:1176-83.

[131] Zhu J, Zhu Y, Wang X. A Hybrid Piezoelectric and Triboelectric Nanogenerator with PVDF Nanoparticles and Leaf-Shaped Microstructure PTFE Film for Scavenging Mechanical Energy. Advanced Materials Interfaces. 2018;5:1700750.

[132] Zhu Y, Yang B, Liu J, Wang X, Chen X, Yang C. An Integrated Flexible Harvester Coupled Triboelectric and Piezoelectric Mechanisms Using PDMS/MWCNT and PVDF. Journal of Microelectromechanical Systems. 2015;24:513-5.

[133] Han M, Zhang XS, Meng B, Liu W, Tang W, Sun X, et al. r-Shaped hybrid nanogenerator with enhanced piezoelectricity. ACS Nano. 2013;7:8554-60.

[134] Suo G, Yu Y, Zhang Z, Wang S, Zhao P, Li J, et al. Piezoelectric and Triboelectric Dual Effects in Mechanical-Energy Harvesting Using BaTiO3/Polydimethylsiloxane Composite Film. ACS Appl Mater Interfaces. 2016;8:34335-41.

[135] Cherumannil Karumuthil S, Rajeev SP, Varghese S. Piezo-tribo nanoenergy harvester using hybrid polydimethyl siloxane based nanocomposite. Nano Energy. 2017;40:487-94.

[136] Qian Y, Kang DJ. Poly(dimethylsiloxane)/ZnO Nanoflakes/Three-Dimensional Graphene Heterostructures for High-Performance Flexible Energy Harvesters with Simultaneous Piezoelectric and Triboelectric Generation. ACS Appl Mater Interfaces. 2018;10:32281-8.

[137] Chowdhury AR, Abdullah AM, Hussain I, Lopez J, Cantu D, Gupta SK, et al. Lithium doped zinc oxide based flexible piezoelectric-triboelectric hybrid nanogenerator. Nano Energy. 2019;61:327-36.

[138] Guo Y, Zhang X-S, Wang Y, Gong W, Zhang Q, Wang H, et al. All-fiber hybrid piezoelectricenhanced triboelectric nanogenerator for wearable gesture monitoring. Nano Energy. 2018;48:152-60. [139] Han M, Zhang X, Liu W, Sun X, Peng X, Zhang H. Low-frequency wide-band hybrid energy harvester based on piezoelectric and triboelectric mechanism. Science China Technological Sciences. 2013;56:1835-41.

[140] Singh HH, Khare N. Flexible ZnO-PVDF/PTFE based piezo-tribo hybrid nanogenerator. Nano Energy. 2018;51:216-22.

[141] Li ZJ, Saadatnia Z, Yang ZB, Naguib H. A hybrid piezoelectric-triboelectric generator for lowfrequency and broad-bandwidth energy harvesting. Energy Conversion and Management. 2018;174:188-97.

[142] Chen T, Xia Y, Liu W, Liu H, Sun L, Lee C. A Hybrid Flapping-Blade Wind Energy Harvester Based on Vortex Shedding Effect. Journal of Microelectromechanical Systems. 2016;25:845-7.

[143] Zhao C, Zhang Q, Zhang W, Du X, Zhang Y, Gong S, et al. Hybrid piezo/triboelectric nanogenerator for highly efficient and stable rotation energy harvesting. Nano Energy. 2019;57:440-9.

[144] Chen Y, Cheng Y, Jie Y, Cao X, Wang N, Wang ZL. Energy harvesting and wireless power transmission by a hybridized electromagnetic-triboelectric nanogenerator. Energy \& Environmental Science. 2019;12:2678-84.

[145] Hou C, Chen T, Li Y, Huang M, Shi Q, Liu H, et al. A rotational pendulum based electromagnetic/triboelectric hybrid-generator for ultra-low-frequency vibrations aiming at human motion and blue energy applications. Nano Energy. 2019;63:103871. 
[146] Gupta RK, Shi Q, Dhakar L, Wang T, Heng CH, Lee C. Broadband Energy Harvester Using Non-linear Polymer Spring and Electromagnetic/Triboelectric Hybrid Mechanism. Sci Rep. 2017;7:41396.

[147] Liu H, Xia Y, Chen T, Yang Z, Liu W, Wang P, et al. Study of a Hybrid Generator Based on Triboelectric and Electromagnetic Mechanisms. IEEE Sensors Journal. 2017;17:3853-60.

[148] Chen X, Guo H, Wu H, Chen H, Song Y, Su Z, et al. Hybrid generator based on freestanding magnet as all-direction in-plane energy harvester and vibration sensor. Nano Energy. 2018;49:51-8.

[149] Ren X, Fan H, Wang C, Ma J, Lei S, Zhao Y, et al. Magnetic force driven noncontact electromagnetictriboelectric hybrid nanogenerator for scavenging biomechanical energy. Nano Energy. 2017;35:23341.

[150] Wang X, Yang Y. Effective energy storage from a hybridized electromagnetic-triboelectric nanogenerator. Nano Energy. 2017;32:36-41.

[151] Seol M-L, Han J-W, Park S-J, Jeon S-B, Choi Y-K. Hybrid energy harvester with simultaneous triboelectric and electromagnetic generation from an embedded floating oscillator in a single package. Nano Energy. 2016;23:50-9.

[152] Salauddin M, Rasel MS, Kim JW, Park JY. Design and experiment of hybridized electromagnetictriboelectric energy harvester using Halbach magnet array from handshaking vibration. Energy Conversion and Management. 2017;153:1-11.

[153] Salauddin M, Toyabur RM, Maharjan P, Park JY. High performance human-induced vibration driven hybrid energy harvester for powering portable electronics. Nano Energy. 2018;45:236-46.

[154] Kiziroglou ME, He C, Yeatman EM. Rolling Rod Electrostatic Microgenerator. IEEE Transactions on Industrial Electronics. 2009;56:1101-8.

[155] Wang X, Wen Z, Guo H, Wu C, He X, Lin L, et al. Fully Packaged Blue Energy Harvester by Hybridizing a Rolling Triboelectric Nanogenerator and an Electromagnetic Generator. ACS Nano. 2016;10:11369-76.

[156] Wang H, Zhu Q, Ding Z, Li Z, Zheng H, Fu J, et al. A fully-packaged ship-shaped hybrid nanogenerator for blue energy harvesting toward seawater self-desalination and self-powered positioning. Nano Energy. 2019;57:616-24.

[157] Hao C, He J, Zhai C, Jia W, Song L, Cho J, et al. Two-dimensional triboelectric-electromagnetic hybrid nanogenerator for wave energy harvesting. Nano Energy. 2019;58:147-57.

[158] Maharjan P, Cho H, Rasel MS, Salauddin M, Park JY. A fully enclosed, 3D printed, hybridized nanogenerator with flexible flux concentrator for harvesting diverse human biomechanical energy. Nano Energy. 2018;53:213-24.

[159] Maharjan P, Toyabur RM, Park JY. A human locomotion inspired hybrid nanogenerator for wristwearable electronic device and sensor applications. Nano Energy. 2018;46:383-95.

[160] Zhang B, Chen J, Jin L, Deng W, Zhang L, Zhang H, et al. Rotating-Disk-Based Hybridized Electromagnetic-Triboelectric Nanogenerator for Sustainably Powering Wireless Traffic Volume Sensors. ACS Nano. 2016;10:6241-7.

[161] Qian J, Jing X. Wind-driven hybridized triboelectric-electromagnetic nanogenerator and solar cell as a sustainable power unit for self-powered natural disaster monitoring sensor networks. Nano Energy. 2018;52:78-87.

[162] Cao R, Zhou T, Wang B, Yin Y, Yuan Z, Li C, et al. Rotating-Sleeve Triboelectric-Electromagnetic Hybrid Nanogenerator for High Efficiency of Harvesting Mechanical Energy. ACS Nano. 2017;11:83708.

[163] Yang H, Wang M, Deng M, Guo H, Zhang W, Yang H, et al. A full-packaged rolling triboelectricelectromagnetic hybrid nanogenerator for energy harvesting and building up self-powered wireless systems. Nano Energy. 2019;56:300-6.

[164] Yang H, Liu W, Xi Y, Lai M, Guo H, Liu G, et al. Rolling friction contact-separation mode hybrid triboelectric nanogenerator for mechanical energy harvesting and self-powered multifunctional sensors. Nano Energy. 2018;47:539-46.

[165] He X, Wen Q, Sun Y, Wen Z. A low-frequency piezoelectric-electromagnetic-triboelectric hybrid broadband vibration energy harvester. Nano Energy. 2017;40:300-7.

[166] He J, Wen T, Qian S, Zhang Z, Tian Z, Zhu J, et al. Triboelectric-piezoelectric-electromagnetic hybrid nanogenerator for high-efficient vibration energy harvesting and self-powered wireless monitoring system. Nano Energy. 2018;43:326-39.

[167] Koh KH, Shi Q, Cao S, Ma D, Tan HY, Guo Z, et al. A self-powered 3D activity inertial sensor using hybrid sensing mechanisms. Nano Energy. 2019;56:651-61. 
[168] Rahman MT, Salauddin M, Maharjan P, Rasel MS, Cho H, Park JY. Natural wind-driven ultracompact and highly efficient hybridized nanogenerator for self-sustained wireless environmental monitoring system. Nano Energy. 2019;57:256-68.

[169] Tan YK, Panda SK. Energy Harvesting From Hybrid Indoor Ambient Light and Thermal Energy Sources for Enhanced Performance of Wireless Sensor Nodes. IEEE Transactions on Industrial Electronics. 2011;58:4424-35.

[170] Yang D, Yin H. Energy Conversion Efficiency of a Novel Hybrid Solar System for Photovoltaic, Thermoelectric, and Heat Utilization. IEEE Transactions on Energy Conversion. 2011;26:662-70.

[171] M. Yusop A, Mohamed R, Mohamed A. Inverse dynamic analysis type of MPPT control strategy in a thermoelectric-solar hybrid energy harvesting system. Renewable Energy. 2016;86:682-92.

[172] Singh M, Singh J, Anshula, Kuchroo P, Bhatia H, Bhagat S, et al. Efficient autonomous solar panel and thermo-electric generator (TEG) integrated hybrid energy harvesting system. 2016 Progress in Electromagnetic Research Symposium (PIERS)2016. p. 1764-8.

[173] Gambier P, Anton SR, Kong N, Erturk A, Inman DJ. Piezoelectric, solar and thermal energy harvesting for hybrid low-power generator systems with thin-film batteries. Measurement Science and Technology. 2011;23:015101.

[174] Chen J, Huang Y, Zhang N, Zou H, Liu R, Tao C, et al. Micro-cable structured textile for simultaneously harvesting solar and mechanical energy. Nature Energy. 2016;1:16138.

[175] Zhang N, Chen J, Huang Y, Guo W, Yang J, Du J, et al. A Wearable All-Solid Photovoltaic Textile. Advanced Materials. 2016;28:263-9.

[176] Bito J, Bahr R, Hester JG, Nauroze SA, Georgiadis A, Tentzeris MM. A Novel Solar and Electromagnetic Energy Harvesting System With a 3-D Printed Package for Energy Efficient Internet-ofThings Wireless Sensors. IEEE Transactions on Microwave Theory and Techniques. 2017;65:1831-42.

[177] Yang F, Du L, Chen W, Li J, Wang Y, Wang D. Hybrid energy harvesting for condition monitoring sensors in power grids. Energy. 2017;118:435-45.

[178] Akan OB, Cetinkaya O, Koca C, Ozger M. Internet of Hybrid Energy Harvesting Things. IEEE Internet of Things Journal. 2018;5:736-46.

[179] Wang C, Li J, Yang Y, Ye F. Combining Solar Energy Harvesting with Wireless Charging for Hybrid Wireless Sensor Networks. IEEE Transactions on Mobile Computing. 2018;17:560-76.

[180] Bowen CR, Taylor J, LeBoulbar E, Zabek D, Chauhan A, Vaish RJE, et al. Pyroelectric materials and devices for energy harvesting applications. 2014;7:3836-56.

[181] Kang M, Yeatman EM. Thermal Energy Harvesting Using Pyroelectric and Piezoelectric Effect. Journal of Physics: Conference Series. 2016;773:012073.

[182] Lee J-H, Lee KY, Gupta MK, Kim TY, Lee D-Y, Oh J, et al. Highly Stretchable PiezoelectricPyroelectric Hybrid Nanogenerator. 2014;26:765-9.

[183] Sebald G, Lefeuvre E, Guyomar D. Pyroelectric energy conversion: Optimization principles. IEEE Transactions on Ultrasonics, Ferroelectrics, and Frequency Control. 2008;55:538-51.

[184] Xu X, Zhang C, Han Q, Chu F. Hybrid energy harvesting from mechanical vibrations and magnetic field. 2018;113:013901.

[185] He W, Yang A, Zhang J, Qu C. Magneto-Electric Hybrid Generator for Simultaneously Harvesting Vibration and Stray Magnetic Field Energy. IEEE Magnetics Letters. 2019;10:1-4.

[186] Lhermet H, Condemine C, Plissonnier M, Salot R, Audebert P, Rosset M. Efficient Power Management Circuit: From Thermal Energy Harvesting to Above-IC Microbattery Energy Storage. IEEE Journal of Solid-State Circuits. 2008;43:246-55.

[187] Bito J, Hester JG, Tentzeris MM. A fully autonomous ultra-low power hybrid RF/photovoltaic energy harvesting system with $-25 \mathrm{dBm}$ sensitivity. 2017 IEEE Wireless Power Transfer Conference (WPTC)2017. p. 1-4.

[188] Challa VR, Prasad MG, Fisher FT. A coupled piezoelectric-electromagnetic energy harvesting technique for achieving increased power output through damping matching. Smart Materials and Structures. 2009;18:095029.

[189] Uluşan H, Chamanian S, Pathirana WPMR, Zorlu Ö, Muhtaroğlu A, Külah H. A triple hybrid micropower generator with simultaneous multi-mode energy harvesting. Smart Materials and Structures. 2017;27:014002.

[190] Yang Z, Erturk A, Zu J. On the efficiency of piezoelectric energy harvesters. Extreme Mechanics Letters. 2017;15:26-37. 
[191] Fu H, Zhou S, Yeatman EM. Exploring coupled electromechanical nonlinearities for broadband energy harvesting from low-frequency rotational sources. Smart Materials and Structures. 2019;28:075001.

[192] Wang H, Jasim A, Chen X. Energy harvesting technologies in roadway and bridge for different applications - A comprehensive review. Applied Energy. 2018;212:1083-94.

[193] Chatterjee P, Bryant M. Aeroelastic-photovoltaic ribbons for integrated wind and solar energy harvesting. Smart Materials and Structures. 2018;27:08LT1.

[194] Jeon S-B, Kim D, Yoon G-W, Yoon J-B, Choi Y-K. Self-cleaning hybrid energy harvester to generate power from raindrop and sunlight. Nano Energy. 2015;12:636-45.

[195] Liu H, Zhang J, Shi Q, He T, Chen T, Sun L, et al. Development of a Thermoelectric and Electromagnetic Hybrid Energy Harvester from Water Flow in an Irrigation System. Micromachines (Basel). 2018;9.

[196] Sultana A, Alam MM, Ghosh SK, Middya TR, Mandal D. Energy harvesting and self-powered microphone application on multifunctional inorganic-organic hybrid nanogenerator. Energy. 2019;166:963-71.

[197] Duan J, Duan Y, Zhao Y, Wang Y, Tang Q, He B. Bifunctional polyaniline electrode tailored hybridized solar cells for energy harvesting from sun and rain. Journal of Energy Chemistry. 2018;27:742-7.

[198] Hailing Fu, Wenzhe Song, Yong Qin, Yeatman EM. Broadband Vibration Energy Harvesting from Underground Trains for Self-Powered Condition Monitoring. PowerMEMS 20192019.

[199] Morbiato T, Borri C, Vitaliani RJAE. Wind energy harvesting from transport systems: A resource estimation assessment. 2014;133:152-68.

[200] Abdelkareem MAA, Xu L, Ali MKA, Elagouz A, Mi J, Guo S, et al. Vibration energy harvesting in automotive suspension system: A detailed review. Applied Energy. 2018;229:672-99.

[201] Xu W, Feng X, Wang J, Luo C, Li J, Ming Z. Energy Harvesting-Based Smart Transportation Mode Detection System via Attention-Based LSTM. IEEE Access. 2019;7:66423-34.

[202] Abdelkareem MA, Xu L, Ali MKA, Elagouz A, Mi J, Guo S, et al. Vibration energy harvesting in automotive suspension system: A detailed review. 2018;229:672-99.

[203] Chong Y-W, Ismail W, Ko K, Lee C-Y. Energy Harvesting For Wearable Devices: A Review. IEEE Sensors Journal. 2019;19:9047-62.

[204] Wang J, He T, Lee C. Development of neural interfaces and energy harvesters towards selfpowered implantable systems for healthcare monitoring and rehabilitation purposes. Nano Energy. 2019;65.

[205] He T, Wang H, Wang J, Tian X, Wen F, Shi Q, et al. Self-Sustainable Wearable Textile Nano-Energy Nano-System (NENS) for Next-Generation Healthcare Applications. Advanced Science. 2019;6:1901437. [206] He T, Shi Q, Wang H, Wen F, Chen T, Ouyang J, et al. Beyond energy harvesting - multi-functional triboelectric nanosensors on a textile. Nano Energy. 2019;57:338-52.

[207] Zhu M, Shi Q, He T, Yi Z, Ma Y, Yang B, et al. Self-Powered and Self-Functional Cotton Sock Using Piezoelectric and Triboelectric Hybrid Mechanism for Healthcare and Sports Monitoring. ACS Nano. 2019;13:1940-52.

[208] Xie L, Cai MJAPL. Increased piezoelectric energy harvesting from human footstep motion by using an amplification mechanism. 2014;105:143901.

[209] Fu H, Cao K, Xu R, Bhouri MA, Martínez-Botas R, Kim S-G, et al. Footstep energy harvesting using heel strike-induced airflow for human activity sensing. 2016 IEEE 13th International Conference on Wearable and Implantable Body Sensor Networks (BSN): IEEE; 2016. p. 124-9.

[210] He C, Zhu W, Chen B, Xu L, Jiang T, Han CB, et al. Smart floor with integrated triboelectric nanogenerator as energy harvester and motion sensor. 2017;9:26126-33.

[211] Siddique ARM, Rabari R, Mahmud S, Van Heyst BJE. Thermal energy harvesting from the human body using flexible thermoelectric generator (FTEG) fabricated by a dispenser printing technique. 2016;115:1081-91.

[212] Nozariasbmarz A, Suarez F, Dycus JH, Cabral MJ, LeBeau JM, Öztürk MC, et al. Thermoelectric generators for wearable body heat harvesting: Material and device concurrent optimization. 2020;67:104265.

[213] Khan N, Kalair A, Abas N, Haider A. Review of ocean tidal, wave and thermal energy technologies. Renewable and Sustainable Energy Reviews. 2017;72:590-604. 
[214] Ellabban O, Abu-Rub H, Blaabjerg F. Renewable energy resources: Current status, future prospects and their enabling technology. Renewable \& Sustainable Energy Reviews. 2014;39:748-64.

[215] Shi Q, Wang H, Wu H, Lee C. Self-powered triboelectric nanogenerator buoy ball for applications ranging from environment monitoring to water wave energy farm. Nano Energy. 2017;40:203-13.

[216] Liu L, Shi Q, Ho JS, Lee C. Study of thin film blue energy harvester based on triboelectric nanogenerator and seashore loT applications. Nano Energy. 2019;66:104167.

[217] Wu Z, Guo H, Ding W, Wang Y-C, Zhang L, Wang ZL. A Hybridized Triboelectric-Electromagnetic Water Wave Energy Harvester Based on a Magnetic Sphere. ACS Nano. 2019.

[218] Chen X, Gao L, Chen J, Lu S, Zhou H, Wang T, et al. A chaotic pendulum triboelectricelectromagnetic hybridized nanogenerator for wave energy scavenging and self-powered wireless sensing system. Nano Energy. 2020;69.

[219] Wang ZL, Jiang T, Xu L. Toward the blue energy dream by triboelectric nanogenerator networks. Nano Energy. 2017;39:9-23.

[220] Qing X, Li W, Wang Y, Sun HJS. Piezoelectric transducer-based structural health monitoring for aircraft applications. 2019;19:545.

[221] Fu H, Khodaei ZS, Aliabadi MHF. An Event-Triggered Energy-Efficient Wireless Structural Health Monitoring System for Impact Detection in Composite Airframes. IEEE Internet of Things Journal. 2019:1-.

[222] Le MQ, Capsal J-F, Lallart M, Hebrard Y, Van Der Ham A, Reffe N, et al. Review on energy harvesting for structural health monitoring in aeronautical applications. 2015;79:147-57.

[223] Ryschkewitsch MG. orce Limited Vibration Testing. USA: NASA; 2012.

[224] Wang Y, Yang Z, Li P, Cao D, Huang W, Inman DJ. Energy harvesting for jet engine monitoring. Nano Energy. 2020;75:104853. 


\section{Table Captions}

Table I. Performance comparison of the PE-EM hybrid energy harvesting systems

Table II. Performance comparison of the PE-TE hybrid energy harvesting systems

Table III. Performance comparison of the EM-TE hybrid energy harvesting systems

Table IV. Performance comparison of the PE-EM-TE hybrid energy harvesting systems

Table V. Performance comparison of other hybrid energy harvesting systems

Table VI. Performance comparison of power management circuits for hybrid energy harvesters 
Table I. Performance comparison of the PE-EM hybrid energy harvesting systems

\begin{tabular}{|c|c|c|c|c|c|}
\hline Reference & Configuration & Piezoelectric & Electromagnetic & Device Size & Output performance and comments \\
\hline $\begin{array}{l}\text { Challa et al. } \\
2009[188]\end{array}$ & Cantilever resonance & $\begin{array}{l}\text { PZT coated, } d_{33} \\
\text { Cantilever }\end{array}$ & Coil/magnet in ASM* & $\begin{array}{l}44 \times 24 \times 30 \\
\mathrm{~mm}^{* *}\end{array}$ & $\begin{array}{l}\sim 332 \mathrm{uW} @ 21.6 \mathrm{~Hz} \text {; the acceleration is not } \\
\text { reported }\end{array}$ \\
\hline $\begin{array}{l}\text { Yang et al. } \\
2010[110]\end{array}$ & $\begin{array}{l}\text { Cantilever } \\
\text { Resonance }\end{array}$ & PZT coated, $d_{31}$ & $\begin{array}{l}\text { Coil/magnet in ASM } \\
\text { \& HSM }\end{array}$ & $22 \times 10 \times 10 \mathrm{~mm}$ & PE: 176 uW \& EM: 0.19 uW @ 310 Hz \& 2.5 g \\
\hline $\begin{array}{l}\text { Mahmoudi et al. } \\
2014 \text { [113] }\end{array}$ & $\begin{array}{l}\text { Fixed-fixed beam } \\
\text { Monostable }\end{array}$ & PZT bimorph & Coil/magnet in ASM & $50 \times 10 \times 15 \mathrm{~mm}$ & $\begin{array}{l}\text { Numerical: } 90 \text { mW @ 0.9g a; bandwidth 152-198 } \\
\text { Hz; No experimental results provided. }\end{array}$ \\
\hline $\begin{array}{l}\text { Xu et al. } \\
2016[115]\end{array}$ & Multi-mode vibration & PZT bimorph & Coil/magnet in ASM & $\begin{array}{l}50 \times 20 \times 80 \\
\mathrm{~mm} * *\end{array}$ & $\begin{array}{l}1.4 \mathrm{~mW} @ 22.6 \mathrm{~Hz} \text { and } 2.82 \mathrm{~mW} @ 25.6 \mathrm{~Hz} \& 2 \mathrm{~m} / \mathrm{s}^{2} \\
\text { Dual-beam structure for multi-mode vibration }\end{array}$ \\
\hline $\begin{array}{l}\text { Toyabur et al. } \\
2018 \text { [109] }\end{array}$ & Multi-mode vibration & PZT coated on Al & Coil/magnet in ASM & $93 \times 30 \times 15 \mathrm{~mm}$ & $\begin{array}{l}\text { PE: } 250 \text { uW \& EM: } 244 \text { uW @ } 17 \mathrm{~Hz} \& 0.4 \mathrm{~g} \\
\text { bandwidth: 12-22 Hz }\end{array}$ \\
\hline $\begin{array}{l}\text { Hamid et al. } \\
2017[116]\end{array}$ & Free-sliding magnet & PZT discs - Impact & Coil/magnet in ASM & $68 \times 39 \times 37 \mathrm{~mm}$ & 50-130 uW; operation conditions not provided. \\
\hline $\begin{array}{l}\text { Fan et al. } \\
2018[117]\end{array}$ & $\begin{array}{l}\text { Free sliding, multi- } \\
\text { direction, bi-stable }\end{array}$ & PZT Cantilever & $\begin{array}{l}\text { Coil/magnet in ASM } \\
\text { Nonlinear levitation }\end{array}$ & $\Phi 14 \times 55 \mathrm{~mm}$ & $\begin{array}{l}\text { EM: } 1.23 \mathrm{~mW} \& \mathrm{PE}: 0.18 \mathrm{~mW} @ 1.5 \mathrm{~g} \& 6.5 \mathrm{~Hz} \\
\text { Bandwidth: } 5-7 \mathrm{~Hz}\end{array}$ \\
\hline $\begin{array}{l}\text { Li et al. } \\
2019[118]\end{array}$ & $\begin{array}{l}\text { Oscillating magnet } \\
\text { Force amplification }\end{array}$ & $\begin{array}{l}\text { PZT fixed-fixed } \\
\text { beam }\end{array}$ & Coil/magnet in HSM & $70 \times 45 \times 20 \mathrm{~mm}$ & 0.33 W peak power @ 0.7 g \& 6.9 Hz \\
\hline $\begin{array}{l}\text { Yang et al. } \\
2019[119]\end{array}$ & $\begin{array}{l}\text { Oscillating magnet } \\
\text { Tri-stable }\end{array}$ & PZT Stack & Coil/magnet in ASM & N/A & No actual prototype developed \\
\hline $\begin{array}{l}\text { Dias et al. } \\
2013[120]\end{array}$ & $\begin{array}{l}\text { Airfoil \& cantilever } \\
\text { Flow induced vibration }\end{array}$ & PZT bimorph & Coil/magnet in HSM & $\mathrm{N} / \mathrm{A}$ & No actual prototype developed \\
\hline $\begin{array}{l}\text { Iqbal et al. } \\
2018[122]\end{array}$ & $\begin{array}{l}\text { Airfoil \& cantilever } \\
\text { Dual-beam structure }\end{array}$ & PZT patch & Coil/magnet in ASM & $85 \times 80 \times 40 \mathrm{~mm}$ & $\begin{array}{l}\text { PE: } 156 \text { uW; EM: } 1.57 \text { mW @ 0.4g \& } 11 \text { Hz } \\
9.1 \text { uW @ 6m/s airflow }\end{array}$ \\
\hline $\begin{array}{l}\text { Khan et al. } \\
2016[121]\end{array}$ & Helmholtz resonator & PZT disc & Coil/magnet in ASM & $\Phi 20 \times 24 \mathrm{~mm}$ & PE: 49 uW; EM: 3.2 uW @ 2.1 kHz \& 130 dB \\
\hline
\end{tabular}

* ASM: Approaching-separation mode; HSM: Horizontal-sliding mode.

** Estimated device overall dimensions. 
Table II. Performance comparison of the PE-TE hybrid energy harvesting systems

\begin{tabular}{|c|c|c|c|c|c|}
\hline $\begin{array}{l}\text { Energy } \\
\text { source }\end{array}$ & Reference & Configuration & Piezoelectric material & Triboelectric material & Maximum output performance \\
\hline \multirow{10}{*}{$\begin{array}{l}\text { Pressing } \\
\text { force }\end{array}$} & $\begin{array}{l}\text { Jung et al. } \\
2015 \text { [129] }\end{array}$ & Arc-shape & $\mathrm{Au} / \mathrm{PVDF} / \mathrm{Au}$ & $\mathrm{Au} / \mathrm{PTFE} / \mathrm{Al}$ & $4.44 \mathrm{~mW} / \mathrm{cm}^{2}$ by tapping force of $0.2 \mathrm{~N}$ \\
\hline & $\begin{array}{l}\text { Wang et al. } \\
2017[130]\end{array}$ & Laminate & AZO/P(VDF-TrFE)/AZO & AZO/PDMS/Skin & $0.075 \mathrm{~mW} / \mathrm{cm}^{2}$ by hand force of $5 \mathrm{~N}$ \\
\hline & $\begin{array}{l}\text { Zhu et al. } \\
2018[131]\end{array}$ & Parallel plate & $\mathrm{Cu} / \mathrm{PVDF} / \mathrm{PTFE} / \mathrm{Cu}$ & $\mathrm{Cu} / \mathrm{PTFE} / \mathrm{Cu}$ & $\begin{array}{l}0.15(\mathrm{PEH}) \text { and } 2.75\left(\text { TENG) } \mathrm{mW} / \mathrm{cm}^{2} \text { by acceleration }\right. \\
\text { of } 5 \mathrm{~m} / \mathrm{s}^{2}\end{array}$ \\
\hline & $\begin{array}{l}\text { Zhu et al. } \\
2015[132]\end{array}$ & Parallel plate & Al/PVDF/Al & Al/PDMS/MWCNT-PDMS/Au & $\begin{array}{l}2.27 \times 10^{-3}(\mathrm{PEH}) \text { and } 2.04 \times 10^{-3}(\mathrm{TENG}) \mathrm{mW} / \mathrm{cm}^{2} \text { by } \\
\text { tapping force of } 5 \mathrm{~N}\end{array}$ \\
\hline & $\begin{array}{l}\text { Han et al. } \\
2013[133]\end{array}$ & r-shape & $\mathrm{Al} / \mathrm{PVDF} / \mathrm{Al}$ & Al/PDMS/ITO & $\begin{array}{l}10.95(\mathrm{PEH}) \text { and } 2.04 \text { (TENG) } \mathrm{mW} / \mathrm{cm}^{3} \text { by periodic } \\
\text { force of } 5 \mathrm{~Hz}\end{array}$ \\
\hline & $\begin{array}{l}\text { Suo et al. } \\
2016[134]\end{array}$ & Laminate & \multicolumn{2}{|l|}{ PET/ITO/BTO+PDMS/Cu } & N/A \\
\hline & $\begin{array}{l}\text { Karumuthil et } \\
\text { al. } 2017 \text { [135] }\end{array}$ & Laminate & \multicolumn{2}{|c|}{$\mathrm{Cu} / \mathrm{ZnO}+\mathrm{EGO}+\mathrm{MWCNT+PDMS} / \mathrm{Cu}$} & $\mathrm{N} / \mathrm{A}$ \\
\hline & $\begin{array}{l}\text { Qian et al. } \\
2018[136]\end{array}$ & Laminate & \multicolumn{2}{|c|}{$\mathrm{Au} / \mathrm{ZnO} \mathrm{NFs}+3 \mathrm{D} \mathrm{Gr}+\mathrm{PDMS} / \mathrm{Ni}$} & $6.22 \mathrm{~mW} / \mathrm{cm}^{2}$ by vertical force of $7 \mathrm{~N}$ \\
\hline & $\begin{array}{l}\text { Chowdhury et } \\
\text { al. } 2019 \text { [137] }\end{array}$ & Laminate & \multicolumn{2}{|c|}{ Al/PTFE+PDMS/Li-ZnO+PVDF+MWCNT/Ag } & N/A \\
\hline & $\begin{array}{l}\text { Guo et al. } \\
2018[138]\end{array}$ & Laminate & \multicolumn{2}{|c|}{$\begin{array}{l}\text { Conductive fabrics/silk fibroin/PVDF } \\
\text { nanofiber/conductive fabrics }\end{array}$} & $0.31 \mathrm{~mW} / \mathrm{cm}^{2}$ by vertical force of $25.7 \mathrm{~N}$ \\
\hline \multirow{2}{*}{ Vibration } & $\begin{array}{l}\text { Han et al. } \\
2013 \text { [139] }\end{array}$ & $\begin{array}{l}\text { Cantilever } \\
\text { stopper }\end{array}$ & $\mathrm{Al} / \mathrm{PVDF} / \mathrm{Al}$ & Al/PDMS/ITO & N/A \\
\hline & $\begin{array}{l}\text { Li et al. } \\
2018[141]\end{array}$ & Truss-stopper & $\mathrm{Ag} / \mathrm{PZT}-5 \mathrm{~J} / \mathrm{Ag}$ & Al/PTFE/Nylon/Al & $\begin{array}{l}19.6 \mathrm{~mW} \text { at acceleration of } 1 \mathrm{~g} \mathrm{(} 14 \text { and } 5.7 \mathrm{~mW} \text { for } \\
\text { PEH and TENG, separately) }\end{array}$ \\
\hline Rotation & $\begin{array}{l}\text { Zhao et al. } \\
2019 \text { [143] }\end{array}$ & Rotational blade & $\begin{array}{l}\mathrm{Au} / \mathrm{P}(\mathrm{VDF}-\mathrm{TrFE}) / \mathrm{Au} \\
/ \mathrm{P}(\mathrm{VDF}-\mathrm{TrFE}) / \mathrm{Au}\end{array}$ & $\mathrm{Al} / \mathrm{PTFE} / \mathrm{Au}$ & $10.88 \mathrm{~mW}$ at rational speed of $100 \mathrm{rpm}$ \\
\hline Wind flow & $\begin{array}{l}\text { Chen et al. } \\
2016[142]\end{array}$ & Flapping blade & $\mathrm{Al} / \mathrm{PVDF} / \mathrm{Al}$ & $\mathrm{Al} / \mathrm{PDMS} / \mathrm{Al}$ & $112(\mathrm{PEH})$ and 76 (TENG) $\mu \mathrm{W}$ at wind speed of $10 \mathrm{~m} / \mathrm{s}$ \\
\hline
\end{tabular}


Table III. Performance comparison of the EM-TE hybrid energy harvesting systems

\begin{tabular}{|c|c|c|c|c|c|}
\hline Energy source & Reference & Configuration & $\begin{array}{l}\text { Electromagnetic } \\
\text { effect }\end{array}$ & Triboelectric effect & Maximum output performance \\
\hline \multirow{2}{*}{ Vibration } & $\begin{array}{l}\text { Gupta et al. } \\
2017[146]\end{array}$ & Spring-mass vertically & Coil/magnet in ASM & ITO/PTFE in CSM & $\begin{array}{l}1.3 \mathrm{uW} / \mathrm{cm}^{3} \text { at } 80 \mathrm{~Hz} \text { and } 2 \mathrm{~g} \text { excitation }\left(40 \mathrm{~cm}^{3} \text { in }\right. \\
\text { overall size })\end{array}$ \\
\hline & $\begin{array}{l}\text { Liu et al. } \\
2017 \text { [147] }\end{array}$ & Spring-mass vertically & Coil/magnet in ASM & $\mathrm{Al} / \mathrm{FEP}$ in CSM & $\begin{array}{l}9.7 \text { and } 37.6 \mathrm{uW} / \mathrm{cm}^{3} \text { for EMEH and TENG at } 23.8 \mathrm{~Hz} \\
\text { and } 1 \mathrm{~g} \text { excitation }\left(29 \mathrm{~cm}^{3} \text { in size*) }\right.\end{array}$ \\
\hline \multirow{2}{*}{$\begin{array}{l}\text { Vibration, } \\
\text { human motion }\end{array}$} & $\begin{array}{l}\text { Chen et al. } \\
2018[148]\end{array}$ & Spring-mass laterally & Coil/magnet in LSM & $\mathrm{Cu} /$ Kapton in CSM & $\begin{array}{l}29.9 \text { and } 0.78 \mathrm{uW} / \mathrm{cm}^{3} \text { for EMEH and TENG }\left(110 \mathrm{~cm}^{3}\right. \\
\text { in overall size* })\end{array}$ \\
\hline & $\begin{array}{l}\text { Salauddin et al. } \\
2017[152]\end{array}$ & Magnetic slider laterally & Coil/magnet in ASM & Al/PDMS in LSM & $381 \mathrm{uW} / \mathrm{cm}^{3}$ at $4.5 \mathrm{~Hz}$ and $0.6 \mathrm{~g}$ \\
\hline $\begin{array}{l}\text { Human } \\
\text { motion }\end{array}$ & $\begin{array}{l}\text { Maharjan et al. } \\
2018[159]\end{array}$ & Magnetic ball laterally & Coil/magnet in ASM & $\mathrm{Al} / \mathrm{PTFE}$ in FTM & $\begin{array}{l}5.14 \mathrm{~mW} / \mathrm{cm}^{3} \text { and } 0.22 \mathrm{uW} / \mathrm{cm}^{3} \text { for EMEH and TENG } \\
\text { at less than } 5 \mathrm{~Hz}\end{array}$ \\
\hline \multirow{3}{*}{ Water wave } & $\begin{array}{l}\text { Wang et al. } \\
2016[155]\end{array}$ & Magnetic slider laterally & Coil/magnet in HSM & $\mathrm{Cu} /$ PTFE in FTM & 1.32 and $1.05 \mathrm{uW} / \mathrm{cm}^{3}$ for EMEH and TENG at $2 \mathrm{~Hz}$ \\
\hline & $\begin{array}{l}\text { Wang et al. } \\
2019 \text { [156] }\end{array}$ & Magnetic roller laterally & Coil/magnet in HSM & $\mathrm{Cu} /$ Silicone in FTM & 9 and $0.8 \mathrm{~mW}$ for EMEH and TENG at $2 \mathrm{~Hz}$ \\
\hline & $\begin{array}{l}\text { Hao et al. } \\
2019 \text { [157] }\end{array}$ & Magnetic roller laterally & Coil/magnet in HSM & $\mathrm{Cu} /$ Silicone in FTM & $\begin{array}{l}39.4 \text { and } 0.21 \mathrm{uW} / \mathrm{cm}^{3} \text { for EMEH and TENG at } 2.4 \mathrm{~Hz} \\
\left(378.4 \mathrm{~cm}^{3} \text { in overall size }{ }^{*}\right)\end{array}$ \\
\hline Wind flow & $\begin{array}{l}\text { Wang et al. } \\
2017[150]\end{array}$ & Vibration film vertically & Coil/magnet in ASM & $\mathrm{Cu} / \mathrm{FEP}$ in CSM & $\begin{array}{l}58.3 \text { and } 39.6 \mathrm{uW} / \mathrm{cm}^{3} \text { for EMEH and TENG at wind } \\
\text { speed of } 18 \mathrm{~m} / \mathrm{s}\left(42.9 \mathrm{~cm}^{3} \text { in overall size*) }\right.\end{array}$ \\
\hline Rotation & $\begin{array}{l}\text { Chen et al. } \\
2019 \text { [144] }\end{array}$ & Rotating disk & Coil/magnet in HSM & $\mathrm{Au} / \mathrm{PTFE}$ in FTM & $\begin{array}{l}176.9 \text { and } 111.6 \mathrm{uW} / \mathrm{cm}^{3} \text { for EMEH and TENG at } 900 \\
\mathrm{rpm}\left(1230.9 \mathrm{~cm}^{3} \text { in overall size*) }\right.\end{array}$ \\
\hline \multirow{2}{*}{$\begin{array}{l}\text { Rotation, } \\
\text { wind flow }\end{array}$} & $\begin{array}{l}\text { Qian et al. } \\
2018[161]\end{array}$ & Rotating sleeve & Coil/Magnet in HSM & $\mathrm{Cu} / \mathrm{PTFE}$ in FTM & $\begin{array}{l}0.34 \text { and } 2.13 \mathrm{~mW} \text { for EMEH and TENG at rotation } \\
\text { speed of } 1200 \mathrm{rpm}\end{array}$ \\
\hline & $\begin{array}{l}\text { Cao et al. } \\
2017[162]\end{array}$ & Rotating sleeve & Coil/magnet in HSM & $\mathrm{Cu} / \mathrm{FEP}$ in LSM & $\begin{array}{l}13.8 \mathrm{uW} / \mathrm{cm}^{3} \text { at rotation of } 250 \mathrm{rpm}\left(940.5 \mathrm{~cm}^{3} \text { in }\right. \\
\text { overall size*) }\end{array}$ \\
\hline
\end{tabular}

* Estimated dimensions from references

** ASM: Approaching-separation mode; HSM: Horizontal-sliding mode; CSM: Contact-separation mode; LSM: Lateral-sliding mode; FTM: Freestanding triboelectric-layer mode. 
Table IV. Performance comparison of the PE-EM-TE hybrid energy harvesting systems

\begin{tabular}{|c|c|c|c|c|c|c|}
\hline $\begin{array}{l}\text { Energy } \\
\text { source }\end{array}$ & References & Configuration & Piezoelectric effect & $\begin{array}{l}\text { Electromagnetic } \\
\text { effect }\end{array}$ & $\begin{array}{l}\text { Triboelectric } \\
\text { effect }\end{array}$ & Maximum output performance \\
\hline \multirow{2}{*}{ Vibration } & $\begin{array}{l}\text { He et al. } \\
2017[165]\end{array}$ & $\begin{array}{l}\text { Spring-mass- } \\
\text { stopper }\end{array}$ & $\begin{array}{l}\text { PVDF film in } \\
\text { bending }\end{array}$ & $\begin{array}{l}\text { Coil/magnet in } \\
\text { ASM }\end{array}$ & $\mathrm{Cu} / \mathrm{PVDF}$ in $\mathrm{CSM}$ & $\begin{array}{l}41,66.5 \text { and } 4.6 \mathrm{uW} \text { for PEH, EMEH and } \\
\text { TENG at } 0.5 \mathrm{~g} \text { and } 20 \mathrm{~Hz} \text { excitation }\end{array}$ \\
\hline & $\begin{array}{l}\text { He et al. } \\
2018[166]\end{array}$ & $\begin{array}{l}\text { Magnetic mass- } \\
\text { stopper }\end{array}$ & $\begin{array}{l}\text { PZT sheet in } \\
\text { compression }\end{array}$ & $\begin{array}{l}\text { Coil/magnet in } \\
\text { ASM }\end{array}$ & $\mathrm{Ni} /$ Silicone in SEM & $\begin{array}{l}122 \mathrm{~mW}, 38.4 \mathrm{~mW} \text { and } 78.4 \mathrm{uW} \text { for } \mathrm{PEH} \text {, } \\
\text { EMEH and TENG at } 20 \mathrm{~Hz} \text { excitation }\end{array}$ \\
\hline $\begin{array}{l}\text { Human } \\
\text { motion }\end{array}$ & $\begin{array}{l}\text { Koh et al. } \\
2019[167]\end{array}$ & $\begin{array}{l}\text { Magnetic rolling } \\
\text { ball }\end{array}$ & $\begin{array}{l}\text { PVDF sheet in } \\
\text { compression }\end{array}$ & $\begin{array}{l}\text { Coil/Magnet in } \\
\text { ASM }\end{array}$ & $\mathrm{Al} / \mathrm{PTFE}$ in FTM & $\begin{array}{l}0.19 \mathrm{uW}, 22.4 \mathrm{nW} \text { and } 0.72 \mathrm{uW} \text { for } \mathrm{PEH} \text {, } \\
\text { EMEH and TENG }\end{array}$ \\
\hline Wind flow & $\begin{array}{l}\text { Toyabur Rahman et al. } \\
2019[168]\end{array}$ & $\begin{array}{l}\text { Rotating sleeve } \\
\text { and disk }\end{array}$ & PVDF in bending & $\begin{array}{l}\text { Coil/Magnet in } \\
\text { HSM }\end{array}$ & $\begin{array}{l}\text { Al/PTFE in CSM } \\
\text { and LSM }\end{array}$ & $\begin{array}{l}1.38,268.6 \text { and } 1.67 \mathrm{~mW} \text { for PEH, EMEH } \\
\text { and TENG at wind speed of } 6 \mathrm{~m} / \mathrm{s}\end{array}$ \\
\hline
\end{tabular}


Table V. Performance comparison of the other hybrid energy harvesting systems

\begin{tabular}{|c|c|c|c|c|c|}
\hline Reference & Energy sources & Transduction & Application & Device Size (mm) & Output performance and conditions \\
\hline $\begin{array}{l}\text { Tan et al. } \\
2011[169]\end{array}$ & Light \& thermal & PV \& TEG & $\begin{array}{l}\text { Indoor wireless } \\
\text { sensing }\end{array}$ & $\begin{array}{l}\text { PV: } 55 \times 30 \times 1 \\
\text { TEG: } 20 \times 20 \times 20\end{array}$ & 621 uW @ 1010 lux and $10 \mathrm{~K}$ in indoor conditions \\
\hline $\begin{array}{l}\text { Gambier et al. } \\
2011[173]\end{array}$ & $\begin{array}{l}\text { Vibration, light } \\
\text { \& thermal }\end{array}$ & $P E, P V \&$ TEG & Condition monitoring & $93 \times 25 \times 1.5$ & $\begin{array}{l}\text { TEG: } 6.6 \mathrm{~mW} @ \Delta 31{ }^{\circ} \mathrm{C} ; \mathrm{PV}: 12.5 \mathrm{~mW} @ 223 \mathrm{~W} / \mathrm{m}^{2} \text {; } \\
\text { PE: } 0.49 \mathrm{~mW} @ 0.5 \mathrm{~g} \& 56.4 \mathrm{~Hz}\end{array}$ \\
\hline $\begin{array}{l}\text { Chen et al. } \\
2016[174]\end{array}$ & Solar \& mechanical & TE \& PV & Textile, wearables & $50 \times 40 \times 0.32$ & $0.5 \mathrm{~mW} @ 18 \mathrm{~mW} / \mathrm{cm}^{2}$ light \& walking conditions \\
\hline $\begin{array}{l}\text { Bito et al. } \\
2017[176]\end{array}$ & Solar \& EM & PV \& RF & Autonomous RF & $\begin{array}{l}\text { EM: } 47 \times 47 \times 20 \\
\text { PV: } 114 \times 24\end{array}$ & $\begin{array}{l}\text { PV: } 93 \mathrm{~mW} @ 100 \mathrm{mw} / \mathrm{cm}^{2} \text { irradiance; } \\
\text { RF: } 28 \mathrm{uW} @-17 \mathrm{dBm}\end{array}$ \\
\hline $\begin{array}{l}\text { Yang et al. } \\
2017[177]\end{array}$ & $\begin{array}{l}\text { EM, thermal \& } \\
\text { Vibration }\end{array}$ & EM, TEG \& PE & $\begin{array}{l}\text { Condition monitoring } \\
\text { of smart grids }\end{array}$ & $\begin{array}{l}\text { EM: } 140 \times 20 \times 50 \\
\text { TEG: } 500 \times 82 \times 10 \mathrm{~cm} \\
\text { PE: } 90 \times 17 \times 0.8\end{array}$ & $\begin{array}{l}\text { EM: } 0.7-366 \mathrm{~mW} \text { at wire current of } 100 \mathrm{~A} \text { to } 800 \mathrm{~A} \\
\text { TEG: } 12.9 \mathrm{~mW} \text { to } 1.98 \mathrm{~W} \text { at wire current of } 500 \mathrm{~A} \text { to } 2000 \mathrm{~A} \\
\text { PE: } 0.63 \mathrm{~mW} \text { at } 1 \mathrm{~g} \text { and } 100 \mathrm{~Hz} \text {. }\end{array}$ \\
\hline $\begin{array}{l}\text { Kang et al. } \\
2020[105]\end{array}$ & Thermal \& vibration & PE \& Pyro & Wireless electronics & $70 \times 10 \times 0.7$ & $0.4 \mathrm{uW} @ 15 \mathrm{~K}$ and $0.02 \mathrm{~Hz}$ vibration \\
\hline $\begin{array}{l}\text { Lee } \text { et al. } \\
2014[182]\end{array}$ & Thermal \& vibration & PE \& Pyro & Wearable devices & $\begin{array}{l}\mathrm{N} / \mathrm{A} \text {, layer thickness } \\
0.7 \mu \mathrm{m}\end{array}$ & $\begin{array}{l}400 \mathrm{mV} \text { and } 100 \mathrm{~mA} \text { from } 293 \mathrm{~K} \text { to } 323 \mathrm{~K} \\
<1 \mathrm{~V} \text { and }<100 \mathrm{nA} \text { in Stretch-Compress-Release Motions }\end{array}$ \\
\hline $\begin{array}{l}\text { Xu et al. } \\
2018[184]\end{array}$ & Vibration \& magnetic & PE with magnet & General sensing & $150 \times 30 \times 1$ & $50 \mathrm{uW} @ 0.1 \mathrm{~g}, 40 \mathrm{~Hz}$ and $2 \mathrm{Gs}$. \\
\hline
\end{tabular}

* TEG: Thermoelectric Generation; PE: Piezoelectric; EM: Electromagnetic; Pyro: Pyroelectric; PV: Photovoltaic; RF: Radio Frequency. 
Table VI. Performance comparison of power management circuits for hybrid energy harvesters

\begin{tabular}{|c|c|c|c|c|c|c|c|}
\hline References & $\begin{array}{c}\text { Bandyopadhyay \& } \\
\text { Chandrakasan } \\
2012[128] \\
\end{array}$ & $\begin{array}{l}\text { Uluşan et al. } \\
2017 \text { [189] }\end{array}$ & $\begin{array}{l}\text { Katic et al. } \\
2018 \text { [127] }\end{array}$ & $\begin{array}{c}\text { Tan and } \\
\text { Panda } \\
2011[169] \\
\end{array}$ & $\begin{array}{l}\text { Bito et al. } \\
2017 \text { [187] }\end{array}$ & $\begin{array}{l}\text { Lhermet et al. } \\
2008 \text { [186] }\end{array}$ & $\begin{array}{l}\text { Chamanian et al. } \\
2019 \text { [126] }\end{array}$ \\
\hline $\begin{array}{l}\text { Fabrication } \\
\text { Technology }\end{array}$ & 0.35 um CMOS & 0.18 um CMOS & 0.18 um & $\begin{array}{c}\text { PCB with } \\
\text { off-the-shelf }\end{array}$ & $\begin{array}{l}\text { Off-the-shelf } \\
\text { component }\end{array}$ & $\begin{array}{c}0.35 \text { um (TEG) } \\
0.18 \text { um (RF) }\end{array}$ & 0.18 um HV \\
\hline Energy Source & $\begin{array}{l}\text { Thermal, solar } \\
\text { and vibration }\end{array}$ & $\begin{array}{l}\text { EM } \\
\text { PE } \\
\text { PV }\end{array}$ & $\begin{array}{l}\text { GBFC } \\
\text { TEG }\end{array}$ & $\begin{array}{l}\text { PV } \\
\text { TEG }\end{array}$ & $\begin{array}{l}\text { RF } \\
\text { PV }\end{array}$ & $\begin{array}{l}\text { TEG } \\
\text { RF }\end{array}$ & $\begin{array}{l}\mathrm{PE} \\
\mathrm{EM}\end{array}$ \\
\hline $\begin{array}{l}\text { Excitation } \\
\text { Frequency }\end{array}$ & $\begin{array}{l}\text { PV \& TEG: DC } \\
\text { PE: NA }\end{array}$ & $\begin{array}{l}\text { PE: } 282 \mathrm{~Hz} \\
\text { EM: } 2-3 \mathrm{~Hz} \\
\text { TEG: DC }\end{array}$ & $\begin{array}{l}\text { GBFC: DC } \\
\text { TEG: DC }\end{array}$ & $\begin{array}{l}\text { PV: DC } \\
\text { TEG: DC }\end{array}$ & $\begin{array}{l}\text { RF: AC } \\
\text { PV: DC }\end{array}$ & $\begin{array}{l}\text { TEG: DC } \\
\text { RF: NA }\end{array}$ & $\begin{array}{l}\text { PE: } 20-500 \mathrm{~Hz} \\
\text { EM: } 2-10 \mathrm{~Hz}\end{array}$ \\
\hline $\begin{array}{l}\text { Minimum } \\
\text { Input Power }\end{array}$ & $\begin{array}{l}\text { PV: } 150 \text { uW } \\
\text { TEG: } 90 \text { uW } \\
\text { PE: } 45 \text { uW }\end{array}$ & $\begin{array}{l}\text { PE: } 4.2 \text { uW } \\
\text { EM: } 1 \text { uW } \\
\text { TEG: NA }\end{array}$ & $\begin{array}{l}\text { GBFC: } 0.5 \mathrm{uW} \\
\text { TEG: } 0.5 \mathrm{uW}\end{array}$ & $\begin{array}{l}\text { PV: } 170 \mathrm{uW} \\
\text { TEG: } 100 \mathrm{uW}\end{array}$ & $\begin{array}{c}\text { RF: NA } \\
\text { PV: } 59 \mathrm{nW}\end{array}$ & $\begin{array}{c}\text { TEG: } 70 \mathrm{uW} \\
\text { RF: NA }\end{array}$ & $\begin{array}{l}\text { PE: }<0.7 \text { uW } \\
\text { EM: } 1 \text { uW }\end{array}$ \\
\hline Output Voltage & $1.9 \mathrm{~V}$ & $0.8-1.25 \mathrm{~V}$ & $1.9 \mathrm{~V}$ & $1.8-3.6 \mathrm{~V}$ & $1.5 \mathrm{~V}$ & $1.75-4.3 \mathrm{~V}$ & $1.1-3.41 \mathrm{~V}$ \\
\hline $\begin{array}{l}\text { Max Conversion } \\
\text { Efficiency* }\end{array}$ & $11 \%-13 \%$ & 29\%@68 uW & 85.5\%@56.4 uW & $\begin{array}{l}90 \% \text { with fixed } \\
\text { reference voltage }\end{array}$ & $\begin{array}{l}\text { RF: } 17.6-24.0 \% \\
\text { PV: } 28 \%\end{array}$ & $\begin{array}{l}78 \% \\
\text { NA }\end{array}$ & 90\%@100 uW \\
\hline
\end{tabular}




\section{Figure Captions}

Figure 1. Energy conversion mechanisms and their typical configurations: (a) piezoelectric, (b) electromagnetic, (c) triboelectric, (d) thermoelectric, and (e) pyroelectric.

Figure 2. Typical structural configurations for PEHs including (a) rectangular [40], (b) triangular [43], (c) taper [44], (d) S-shape [45], (e) arc-shape [46], and (f) flextensional structures [48].

Figure 3. Typical structural configurations for EMEHs including (a)-(d) resonant [50, 53, 54, $56]$ and $(\mathrm{e})-(\mathrm{g})$ rotational structures [57-59].

Figure 4. Typical structural configurations for TENGs including (a)-(b) contact-separation [62, 63], (c)-(e) lateral-sliding [64-66], (f)-(g) single-electrode [67, 68], and (h)-(i) freestanding triboelectric-layer $[70,71]$ modes.

Figure 5. Typical structural configurations for TEHs, including (a) light-to-heat TEH [83], (b) flexible TEH (Reprinted (adapted) with permission from [86]. Copyright (2020) American Chemical Society.), (c) wearable TEH [80], (d) and (e) PCM-based TEHs [88, 89].

Figure 6. Typical structural configurations for pyroelectric energy harvesters. (a) Liquid-metal based pyroelectric harvester [108], (b) pyroelectric harvester for exhaust gas heat recovery [103], (c) pyroelectric harvester for water splitting [100], (d) oscillating heat pipe-based harvester [107] and (e) wind-driven pyroelectric harvester [105].

Figure 8. Configuration illustration of the PE-EM hybrid systems for harvesting airflow (a) [120], (b) [122] and (d) [123] and for collecting acoustic energy (c) [121].

Figure 9. Typical power management circuits for regulating power from PE-EM hybrid systems, including typical function blocks (a), implementation solution (b) and one complete PE-EM solution (c) and (d) [124-126].

Figure 10. Configuration and material illustrations of the PE-TE hybrid systems driven by external force with (a) separated [131] and (b) coexisted [136] piezoelectric and triboelectric effects.

Figure 11. Configuration illustrations of the PE-TE hybrid systems driven by vibration [140, $141]$ and wind flow $[142,143]$.

Figure 12. Resonant structures of the EM-TE hybrid systems, by employing spring-mass [146148], deflected-membrane [150], and magnetic-floating [151, 152] structures.

Figure 13. Non-resonant and rotational structures of the EM-TE hybrid systems, for (a)-(c) water wave energy harvesting [155-157], (d) human wrist-motion harvesting [158], and rotation energy harvesting $[160,161]$.

Figure 14. Configuration illustrations of the PE-EM-TE hybrid systems by using (a) springmass structure [165], (b) magnetic levitation structure [166], (c) non-resonant magnetic rolling ball [167], and (d) windmill structure [168].

Figure 15. Other type of hybrid energy harvesting systems. (a) Hybrid energy harvester from photovoltaic, thermoelectric and hot water energy [170], (b) hybrid solar and mechanical harvester [174], (c) hybrid piezoelectric and pyroelectric harvester [105], (d) stretchable piezoelectric and pyroelectric harvester [182] and (e) hybrid solar and EM harvester [176]. 
Figure 16 Energy sources and application prospect for hybrid energy harvesting systems. 


\section{Figure 1}

Energy conversion mechanisms and their typical configurations: (a) piezoelectric, (b) electromagnetic, (c) triboelectric, (d) thermoelectric, and (e) pyroelectric.

\begin{tabular}{|c|c|c|}
\hline Principle & Config & uration \\
\hline (a) & Cantilever & (a-ii) \\
\hline (b) & $\begin{array}{l}\text { (b-i) } \\
\text { Magnet } \\
\text { Resonant structure } \\
\text { Approaching-separation mode (ASM) }\end{array}$ & $\begin{array}{c}\text { (b-ii) } \\
\text { Magnet } \\
\text { Rotational structure } \\
\text { Hoizontal-sliding mode (HSM) }\end{array}$ \\
\hline \Force & Single-electrode mode (SEM) & Freestanding triboelectric-layer mode (FTM) \\
\hline Thermoelectric & (d-i) & $\begin{array}{c}\text { (d-ii) } \\
\text { Chsulation- Changing Temperat Bridges } \\
\begin{array}{c}\text { Phasechange } \\
\text { material }\end{array}\end{array}$ \\
\hline 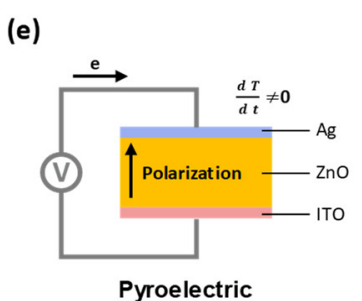 & Changing Temperature Mode & Constant Temperature Gradient Mode \\
\hline
\end{tabular}




\section{Figure 2}

Typical structural configurations for PEHs including (a) rectangular [40], (b) triangular [43], (c) taper [44], (d) S-shape [45], (e) arc-shape [46], and (f) flextensional structures [48].

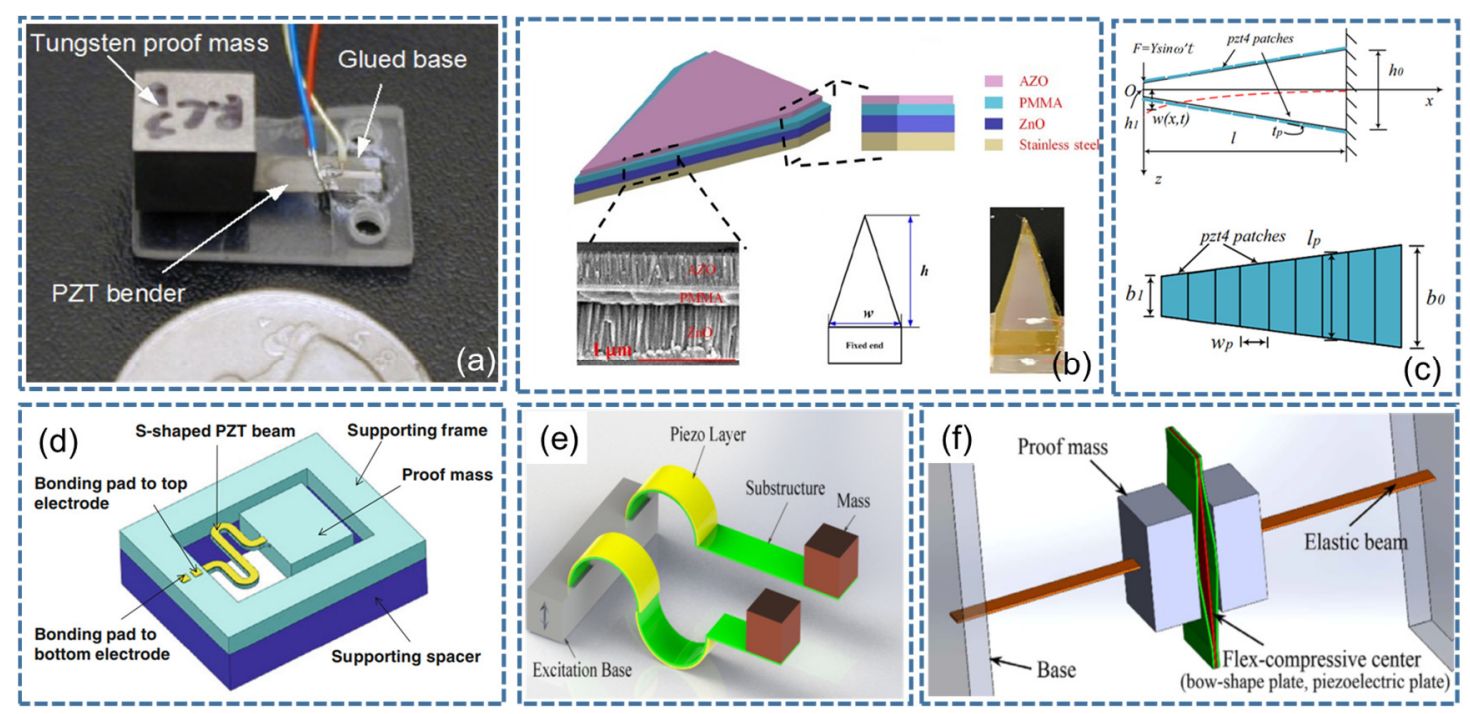




\section{Figure 3}

Typical structural configurations for EMEHs including (a)-(d) resonant $[50,53,54,56]$ and (e)(g) rotational structures [57-59].

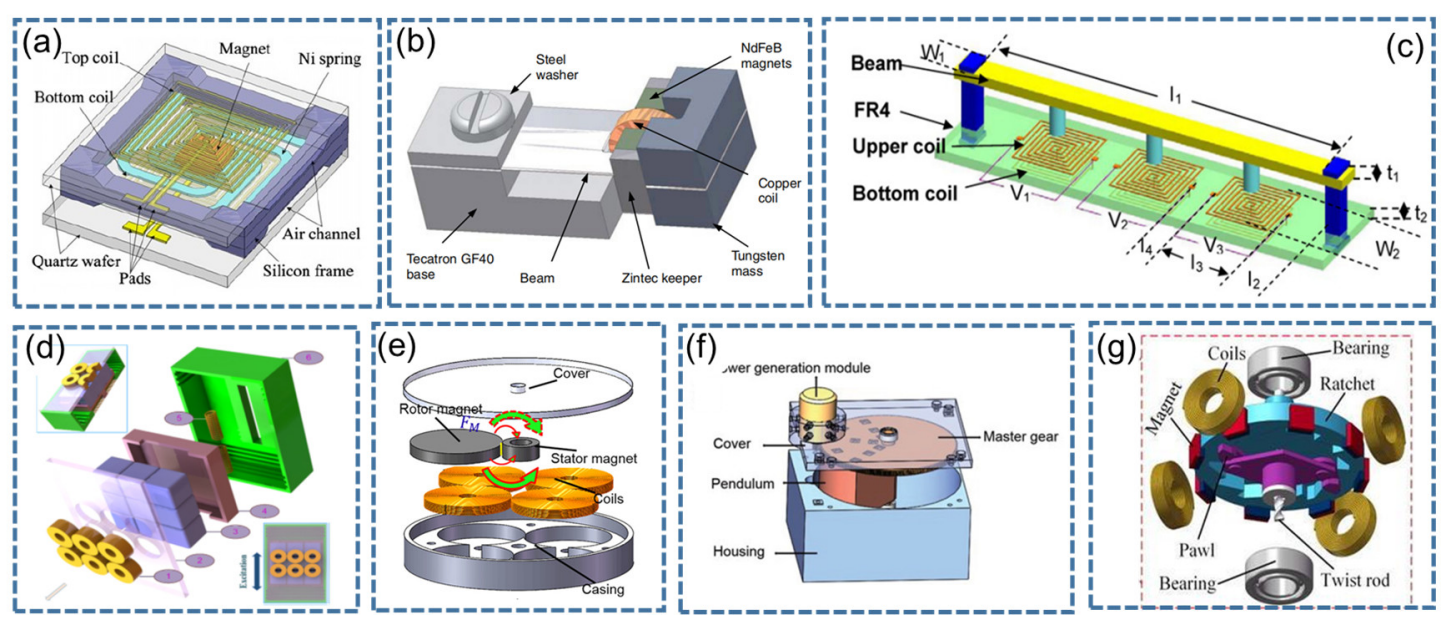




\section{Figure 4}

Typical structural configurations for TENGs including (a)-(b) contact-separation [62, 63], (c)(e) lateral-sliding [64-66], (f)-(g) single-electrode [67, 68], and (h)-(i) freestanding triboelectric-layer $[70,71]$ modes.

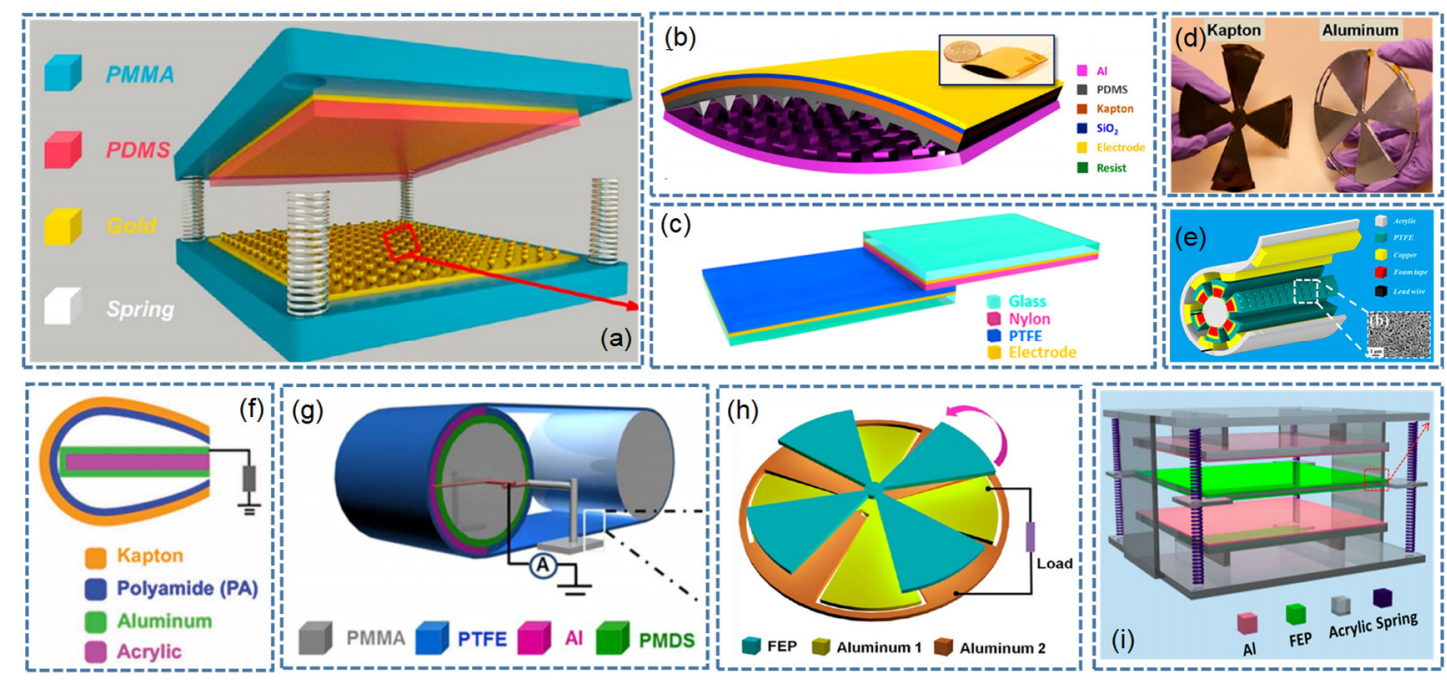




\section{Figure 5}

Typical structural configurations for TEHs, including (a) light-to-heat TEH [83], (b) flexible TEH (Reprinted (adapted) with permission from [86]. Copyright (2020) American Chemical Society.), (c) wearable TEH [80], (d) and (e) PCM-based TEHs [88, 89].

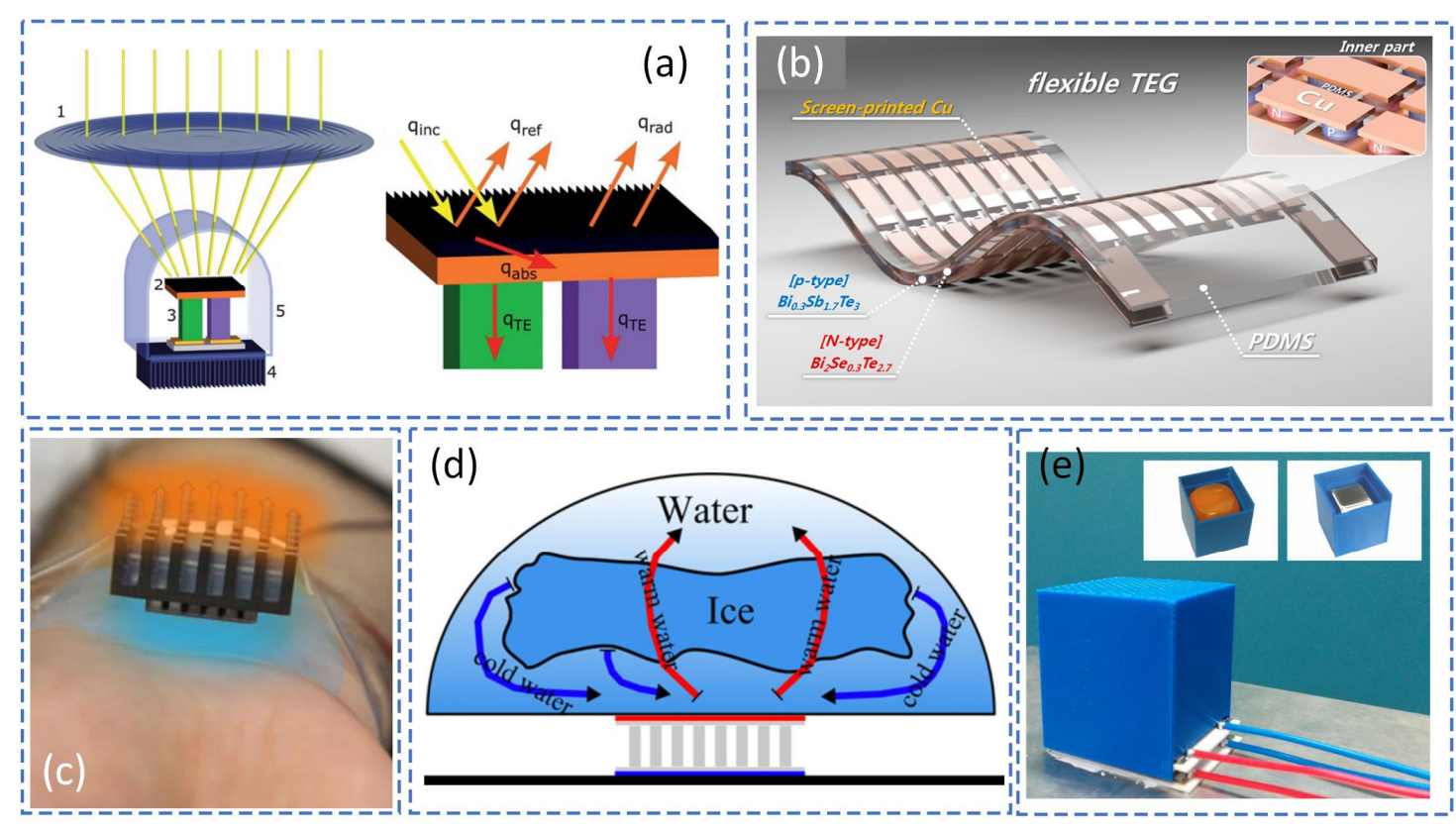




\section{Figure 6}

Typical structural configurations for pyroelectric energy harvesters. (a) Liquid-metal based pyroelectric harvester [108], (b) pyroelectric harvester for exhaust gas heat recovery [103], (c) pyroelectric harvester for water splitting [100], (d) oscillating heat pipe-based harvester [107] and (e) wind-driven pyroelectric harvester [105].
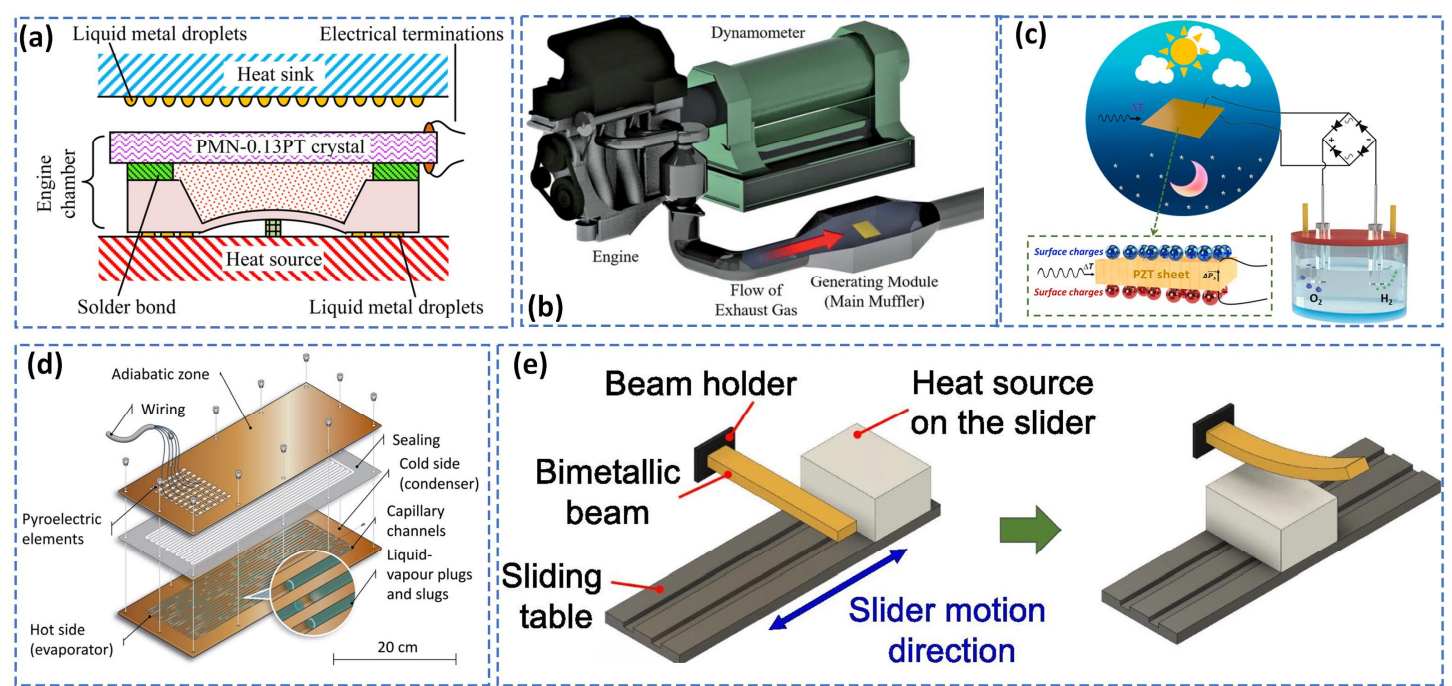


\section{Figure 7}

Configuration illustrations of the PE-EM hybrid systems based on beams (a), (b), (d) and (e) $[109,110,114,115]$ and based on oscillating mass (c), (f) and (g) [117-119].

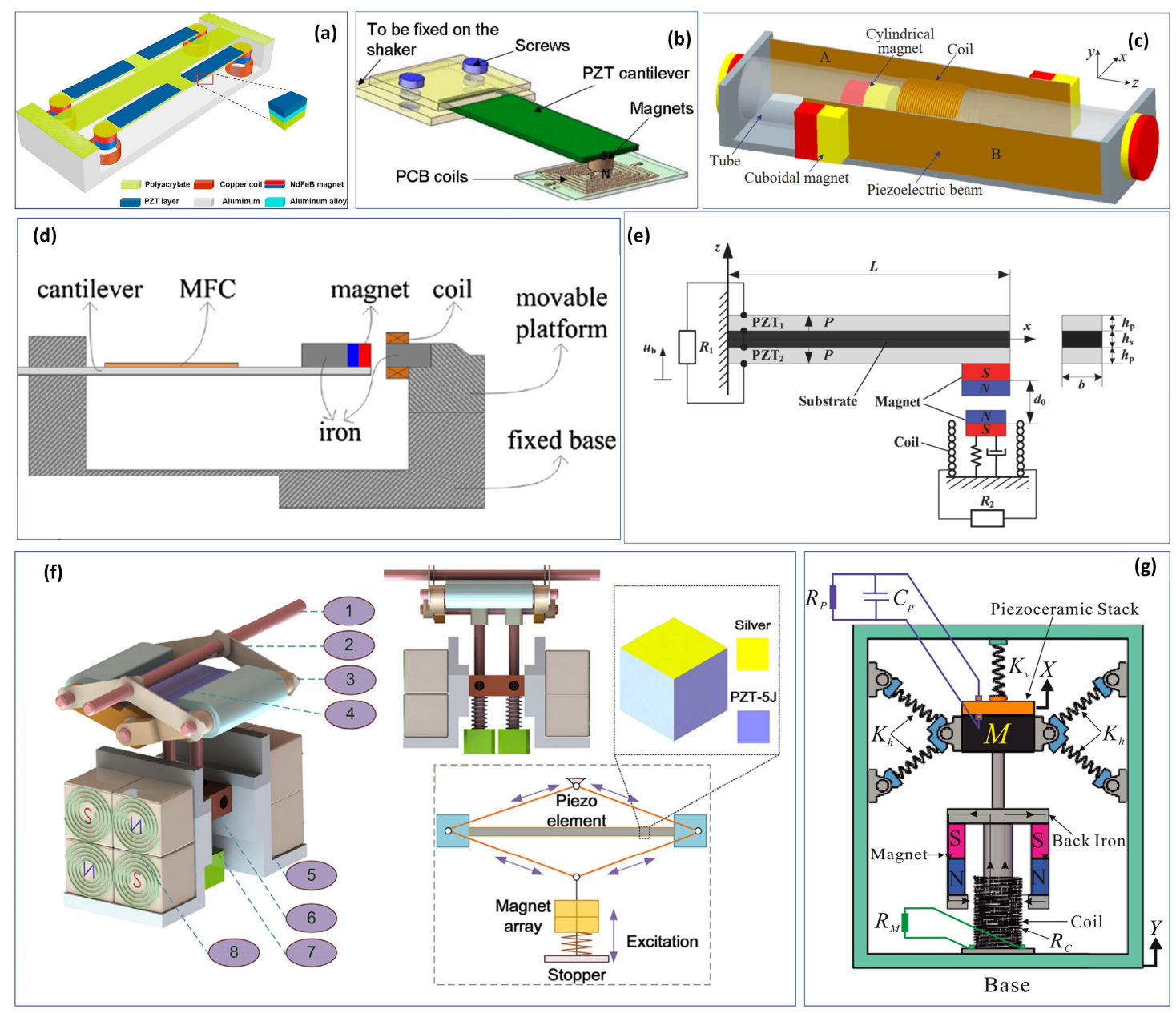




\section{Figure 8}

Configuration illustration of the PE-EM hybrid systems for harvesting airflow (a) [120], (b) [122] and (d) [123] and for collecting acoustic energy (c) [121].
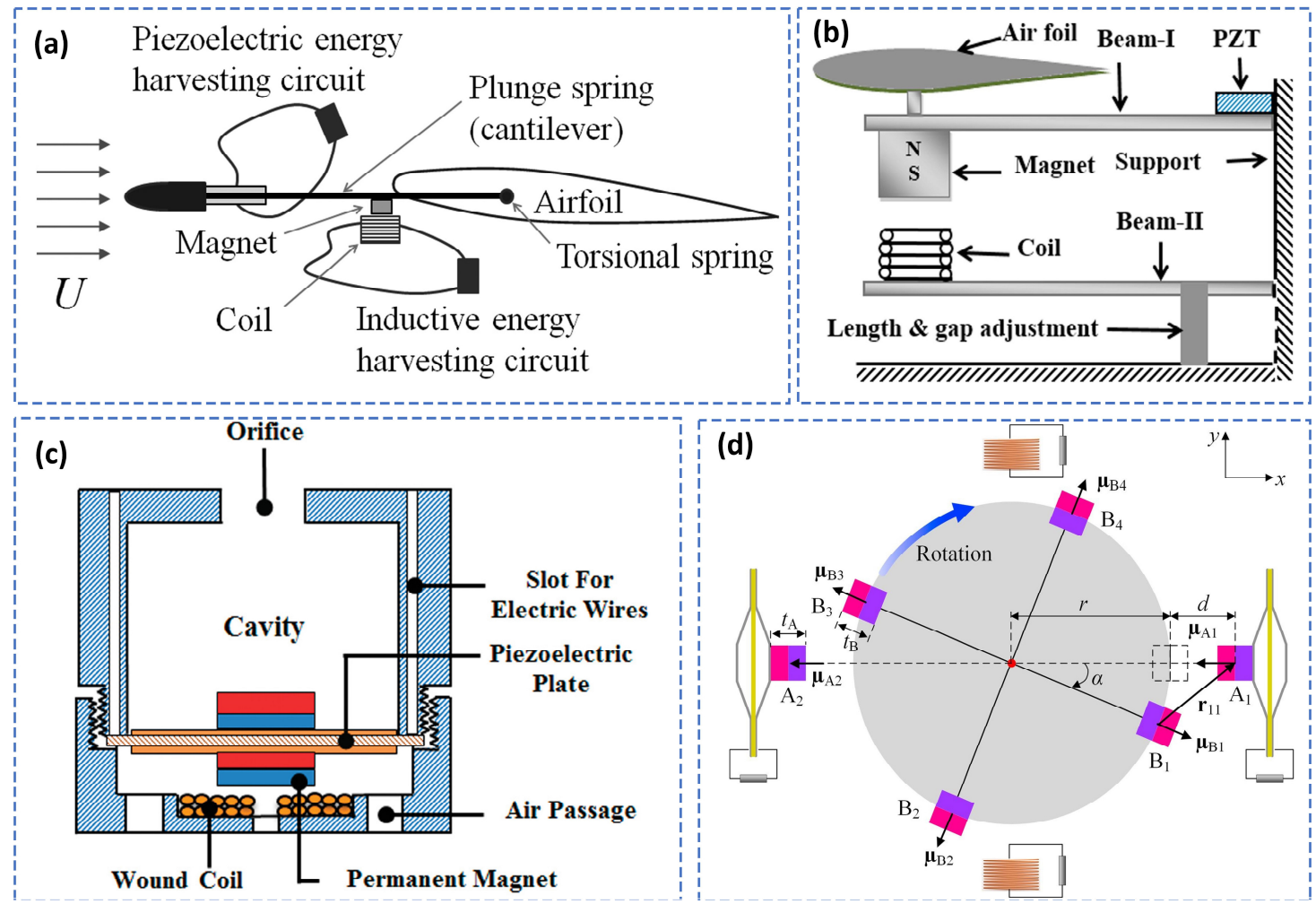


\section{Figure 9}

Typical power management circuits for regulating power from PE-EM hybrid systems, including typical function blocks (a), implementation solution (b) and one complete PE-EM solution (c) and (d) [124-126].

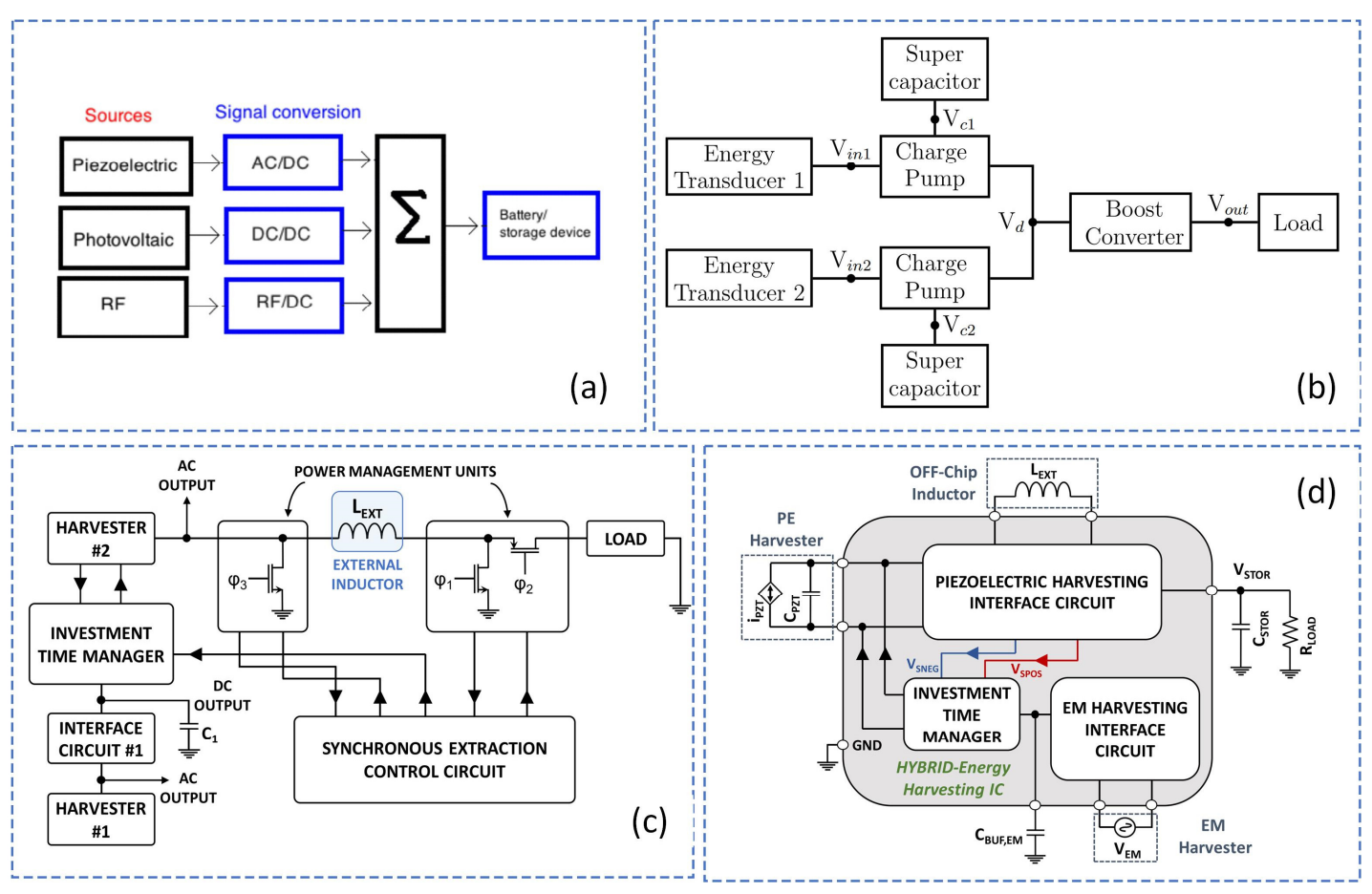




\section{Figure 10}

Configuration and material illustrations of the PE-TE hybrid systems driven by external force with (a) separated [131] and (b) coexisted [136] piezoelectric and triboelectric effects.

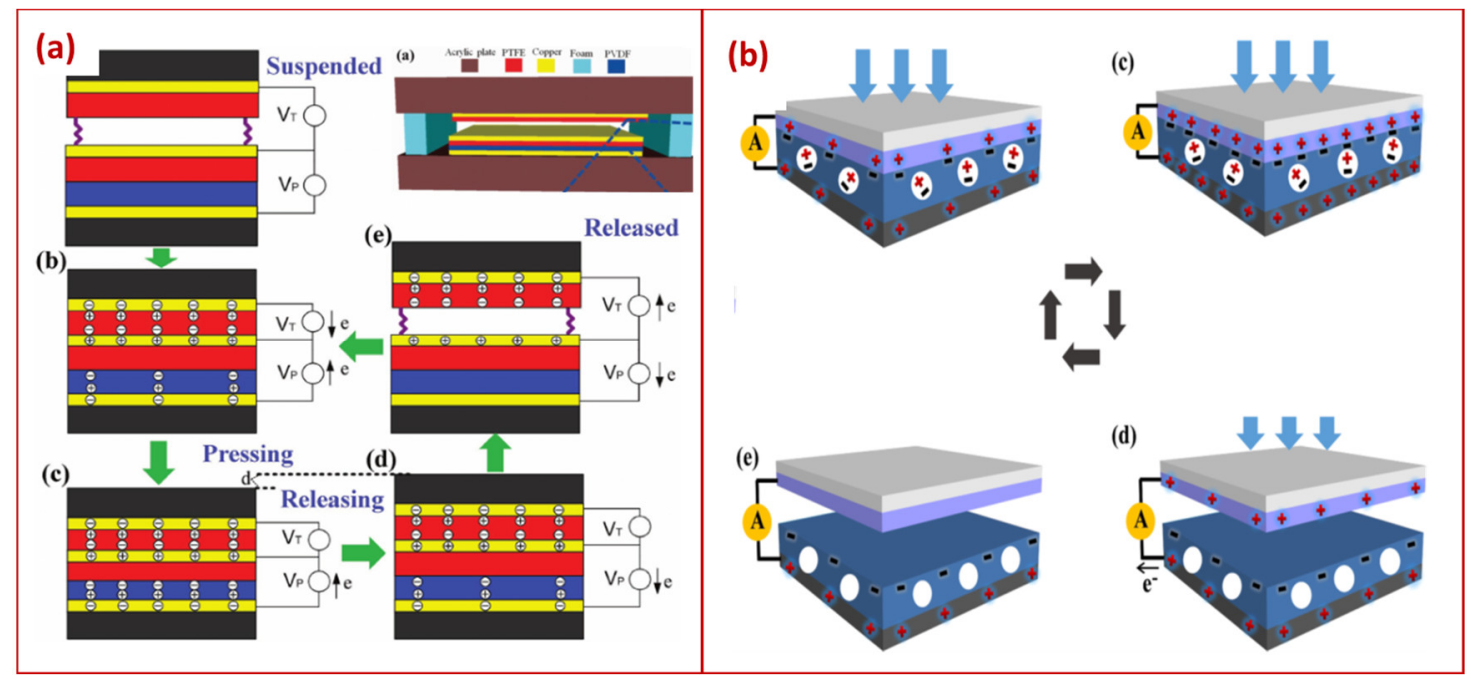




\section{Figure 11}

Configuration illustrations of the PE-TE hybrid systems driven by vibration $[140,141]$ and wind flow $[142,143]$.
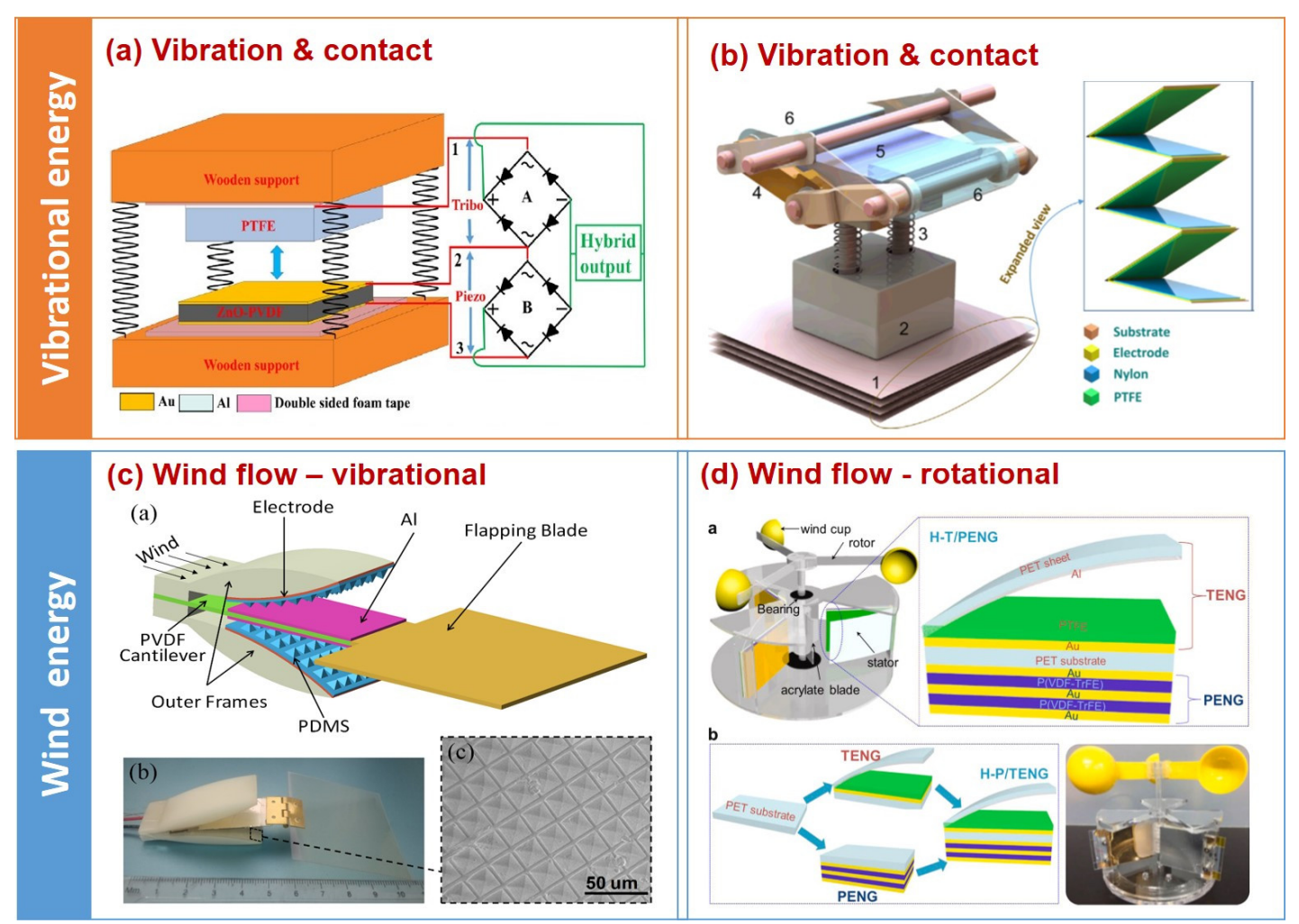


\section{Figure 12}

Resonant structures of the EM-TE hybrid systems, by employing spring-mass [146-148], deflected-membrane [150], and magnetic-floating [151,152] structures.

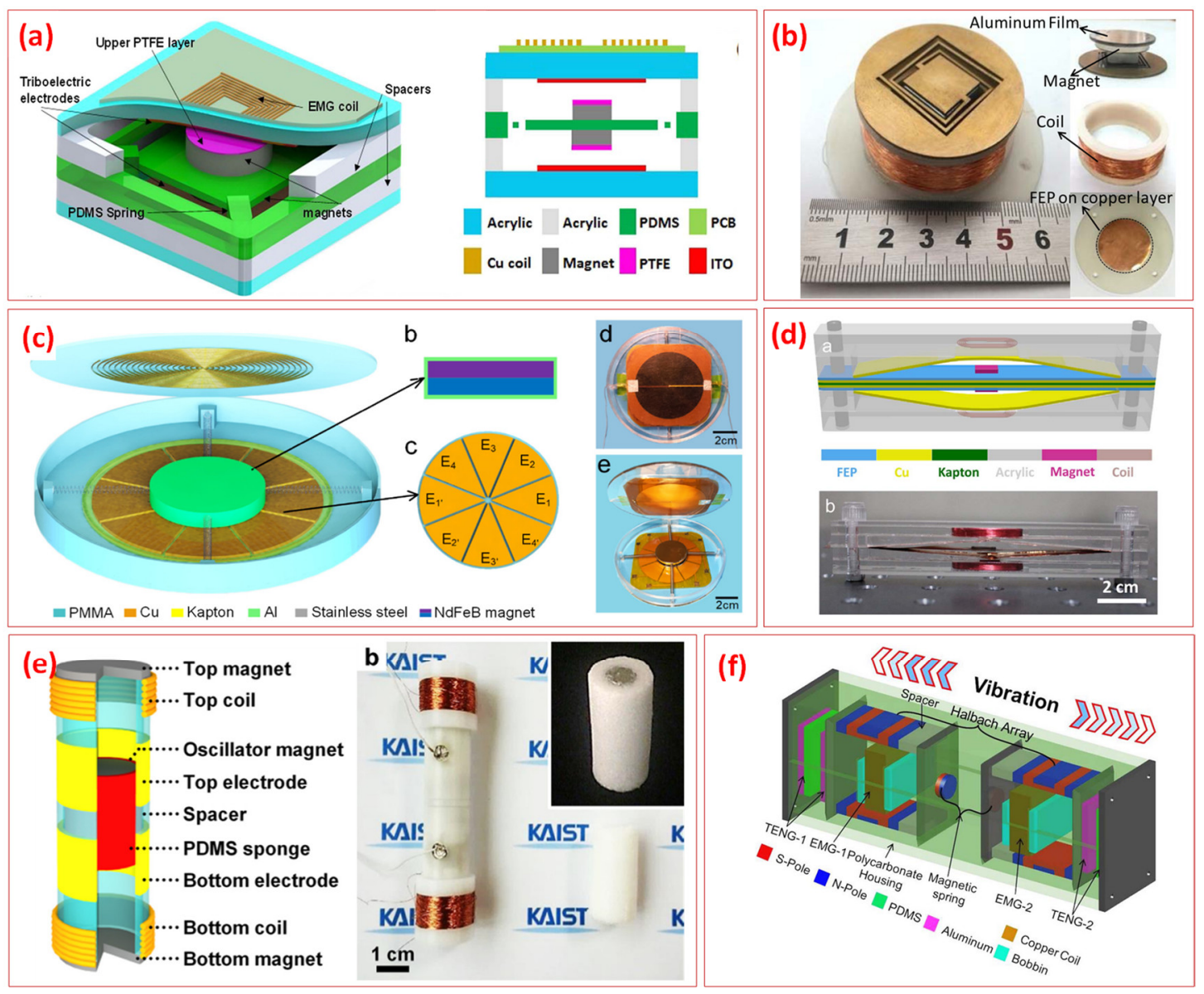




\section{Figure 13}

Non-resonant and rotational structures of the EM-TE hybrid systems, for (a)-(c) water wave energy harvesting [155-157], (d) human wrist-motion harvesting [158], and rotation energy harvesting $[160,161]$.

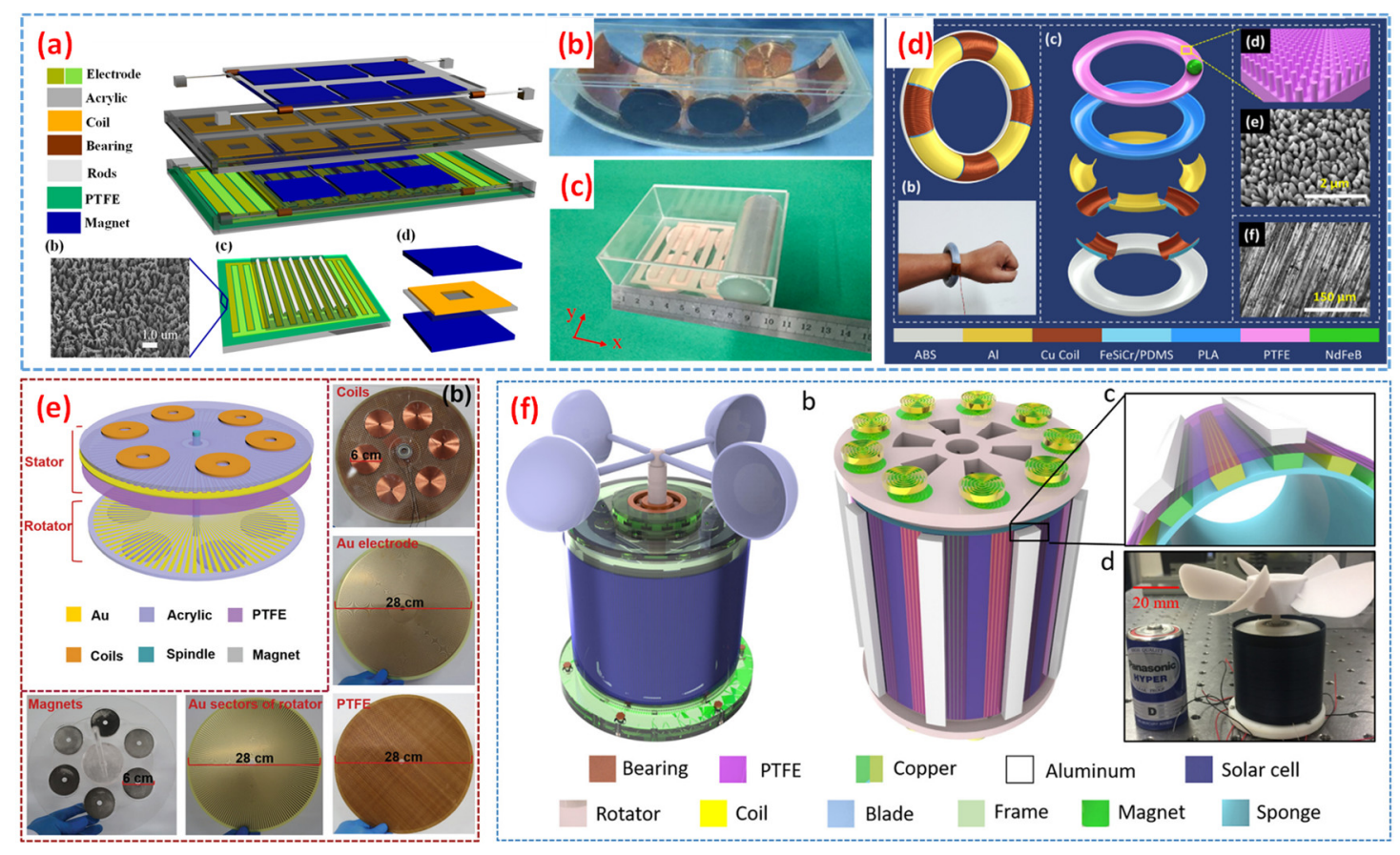




\section{Figure 14}

Configuration illustrations of the PE-EM-TE hybrid systems by using (a) spring-mass structure [165], (b) magnetic levitation structure [166], (c) non-resonant magnetic rolling ball [167], and (d) windmill structure [168].

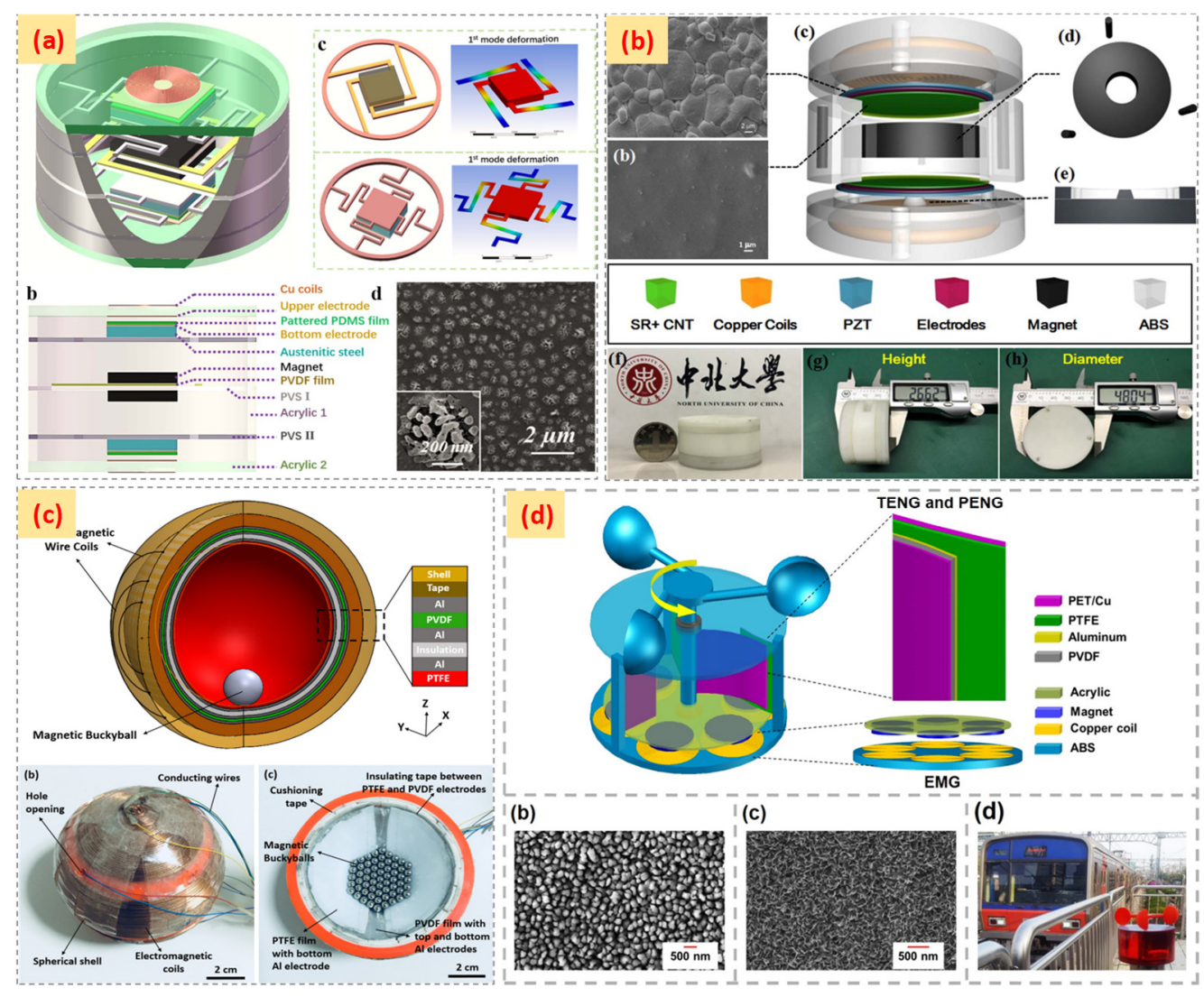




\section{Figure 15}

Other type of hybrid energy harvesting systems. (a) Hybrid energy harvester from photovoltaic, thermoelectric and hot water energy [170], (b) hybrid solar and mechanical harvester [175], (c) hybrid piezoelectric and pyroelectric harvester [105], (d) stretchable piezoelectric and pyroelectric harvester [182] and (e) hybrid solar and EM harvester [176].
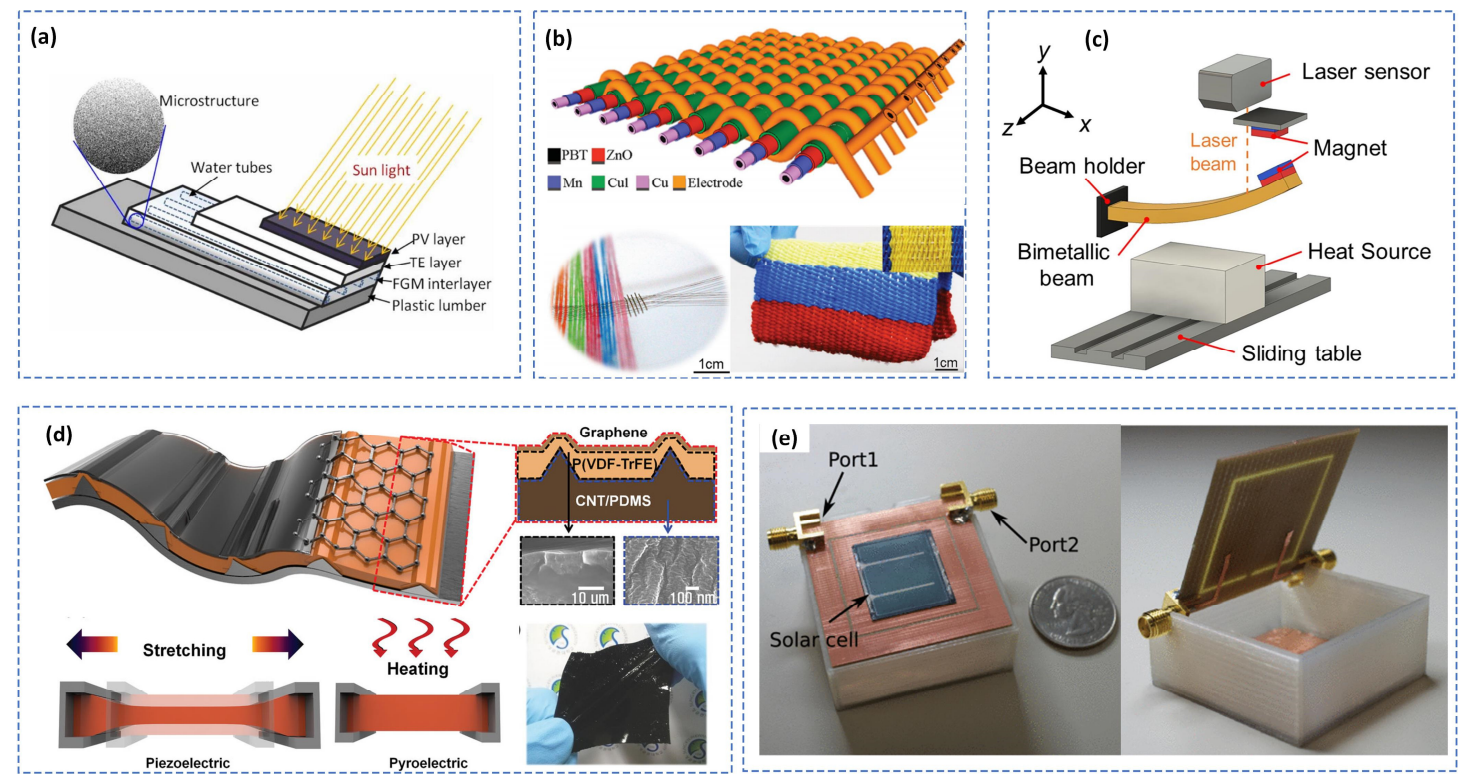
Figure 16

Energy sources and application prospect for hybrid energy harvesting systems.

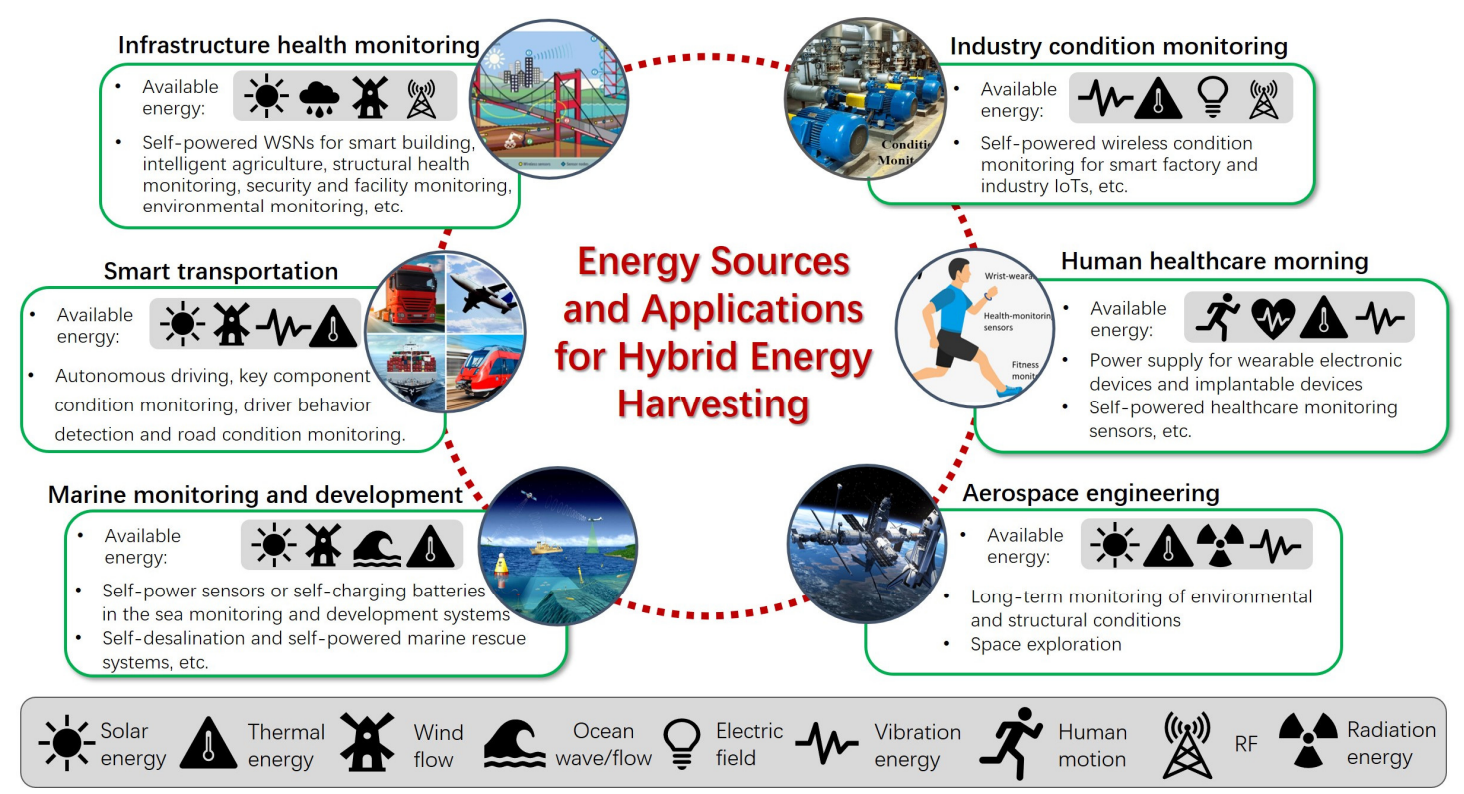

